\title{
An Observational Overview of Solar Flares
}

\author{
L. Fletcher ${ }^{1}$, B. R. Dennis ${ }^{2}$, H. S. Hudson ${ }^{3}$, \\ S. Krucker ${ }^{3}$, K. Phillips ${ }^{4}$, A. Veronig ${ }^{5}$, M. Battaglia ${ }^{1}$, \\ L. Bone ${ }^{4}$, A. Caspi ${ }^{3}$, Q. Chen ${ }^{7}$, P. Gallagher ${ }^{8}$, \\ P. T. Grigis ${ }^{9}$, · H. Ji ${ }^{10,11}$, W. Liu ${ }^{2,12}$, R. O. Milligan ${ }^{2}$, \\ and M. Temmer $5,13,14$
}

\begin{abstract}
We present an overview of solar flares and associated phenomena, drawing upon a wide range of observational data primarily from the RHESSI era. Following an introductory discussion and overview of the status of observational capabilities, the article is split into topical sections which deal with different areas of flare phenomena (footpoints and ribbons, coronal sources, relationship to coronal mass ejections) and their interconnections. We also discuss flare soft X-ray spectroscopy and the energetics of the process. The emphasis is to describe the observations from multiple points of view, while bearing in mind the models that link them to each other and to theory. The present theoretical and observational understanding of solar flares is far from complete, so we conclude with a brief discussion of models, and a list of missing but important observations.
\end{abstract}

Keywords Sun

\section{Contents}

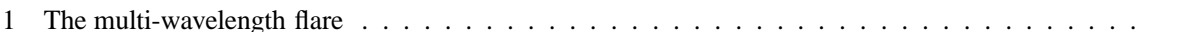

1.1 Flare development . . . . . . . . . . . . . . . . . . .

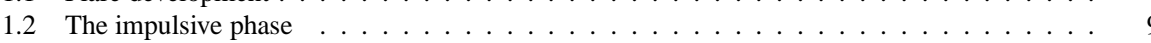

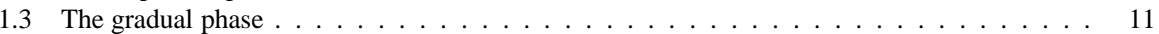

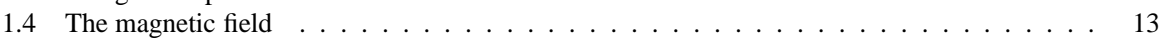

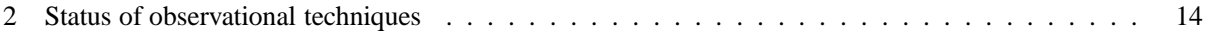

${ }^{1}$ Department of Physics and Astronomy, University of Glasgow, Glasgow G12 8QQ, U. K.

2 NASA Goddard Space Flight Center, Greenbelt, MD USA

3 Space Sciences Laboratory, U.C. Berkeley, CA USA 94720-7450

${ }^{4}$ Mullard Space Science Laboratory, Holmbury St. Mary, Dorking, RH5 CNT, U. K.

5 Institute for Geophysics, Astrophysics and Meteorology, University of Graz, Graz A-8010, Austria

7 Stanford University Physics Department, Varian Building, 382 Via Pueblo Mall, Stanford CA USA 94305

${ }^{8}$ School of Physics, Trinity College Dublin, Dublin 2, Ireland

${ }^{9}$ Harvard-Smithsonian center for Astrophysics, 60 Garden Street, Cambridge MA USA 02138

${ }^{10}$ Purple Mountain Observatory, 2 W. Beijing Rd, Nanjing, 210008, China

11 Big Bear Solar Observatory, New Jersey Institute of Technology, 40386 North Shore Lane, Big Bear City, CA USA 92314

12 Stanford-Lockheed Institute for Space Research, 466 Via Ortega, Cypress Hall, Stanford, CA USA 943054085

13 Hvar Observatory, Faculty of Geodesy, Kaciceva 26, 10000 Zagreb, Croatia

14 Space Research Institute, Austrian Academy of Sciences, Schmiedlstraße 6, A-8042 Graz, Austria 


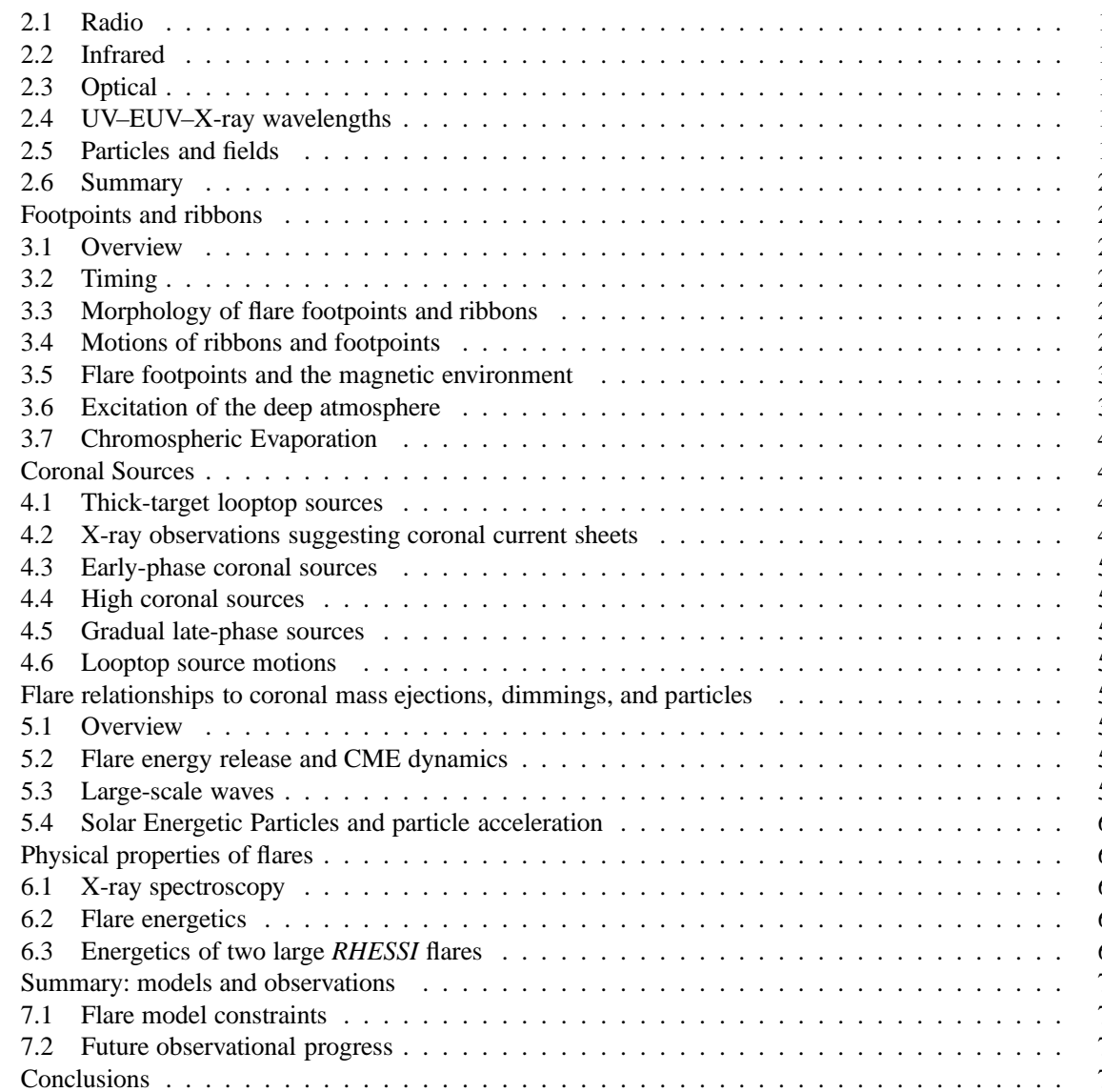

\section{The multi-wavelength flare}

Solar flares are the most powerful magnetic events in the solar system. In tens of minutes they can release in excess of $10^{32} \mathrm{erg}$ of energy. They emit radiation across the entire electromagnetic spectrum, from radio to $\gamma$-rays, and are also intimately associated with the acceleration of particles into interplanetary space and with coronal mass ejections. The flare results from the rapid release of energy previously stored as the inductive magnetic fields due to electrical currents flowing into the corona. The total flare energy is compatible with the amount of magnetic "free" energy (usually defined as the energy stored in the magnetic field that is over and above the energy of the potential magnetic field defined by the same boundaries) inferred to be available in the magnetic active regions (i.e., the coronal connections of a sunspot group) where most flares take place. The magnetic free energy is is hard to evaluate from observations, depending as it does on the magnetic vector field, but in the few cases in which this has been possible (e.g., Metcalf et al. 1995, 2005; Schrijver et al. 2008; Jing et al. 2008), and it is found that the free energy is comparable with that of large flares. Furthermore, the energy budget is difficult to explain from other possible coronal or chromospheric energy sources (e.g., Hudson 2007). So we can conclude that conversion of stored magnetic energy is at the heart of the flare process. 
Table 1.1 GOES and $\mathrm{H} \alpha$ classifications

\begin{tabular}{llll}
\hline $\begin{array}{l}\text { GOES } \\
\text { class }\end{array}$ & $\begin{array}{l}\mathrm{EM}^{a} \\
\mathrm{~cm}^{-3}\end{array}$ & $\begin{array}{l}\mathrm{H} \alpha \\
\text { class }\end{array}$ & $\begin{array}{l}\mathrm{H} \alpha \text { area } \\
\text { Sq. degrees }\end{array}$ \\
\hline $\mathrm{X} 10$ & $10^{51}$ & 4 & 24.7 \\
$\mathrm{X}$ & $10^{50}$ & 3 & 12.4 \\
$\mathrm{M}$ & $10^{49}$ & 2 & 5.1 \\
$\mathrm{C}$ & $10^{48}$ & 1 & 2.0 \\
$\mathrm{~B}$ & $10^{47}$ & $\mathrm{~S}$ & $<2.0$ \\
$\mathrm{~A}$ & $10^{46}$ & $\mathrm{~S}$ & $<2.0$ \\
\hline
\end{tabular}

The term "flare" is normally taken to refer specifically to the electromagnetic radiation of this whole magnetically-driven event, which embodies a significant fraction of the total energy liberated. The total energy released varies from event to event, with many more small events than large events. The distribution of the number of flares as a function of their peak energy, or their total energy, or their duration, is approximately a power law, the gradient of which is a critical factor in understanding the contribution of flare-like heating events to the overall energy budget of the solar corona (e.g., Crosby et al. 1993; Hannah et al. 2011). The primary way of classifying the "importance" of a flare is via its soft X-ray (SXR) flux at 1-8 $\AA$, as measured by GOES (the Geostationary Orbiting Environmental Satellites). Flares are classified into X, M, C, B and A flares, with X corresponding to GOES flux in excess of $10^{-4} \mathrm{~W} \mathrm{~m}^{-2}$ at Earth, and successive classifications decreasing in decades. Table 1 puts the $\mathrm{X}$-ray class of a flare in the context of the older classification based on $\mathrm{H} \alpha$ area (data from Thomas \& Teske 1971).

The majority of the radiative flare energy emerges at visible and ultraviolet wavelengths (Woods et al. 2006). Where a bolometric measurement is possible, i.e., in the most energetic flares, we find that the radiated optical luminosity is comparable to the kinetic energy of the coronal mass ejection (see Section 6.2), and also to the the energy of the accelerated electrons as inferred from the hard X-radiation (HXR) (Fletcher et al. 2007) under the assumptions of the collisional thick-target model (Brown 1971). A lot of emphasis has been placed on hard X-rays (HXRs) in understanding the flare energization process - see Holman et al. (2011) - despite the fact that energetically they represent only a small fraction of the total radiation. However, as HXRs result mainly from the well-understood bremsstrahlung radiation process, and the sources are optically thin, it is relatively straightforward to interpret them. The HXR emission is thus a powerful diagnostic for flare electrons, compared to longer wavelength, optically thick radiation, and the measurement of flare HXRs has been a primary goal of the Reuven Ramaty High Energy Solar Spectroscopic Imager (RHESSI, Lin et al. 2002). However, hard X-rays alone give only a restricted view of the overall configuration, development and energetics of a flare, and of its relationship to accompanying dynamical processes. The aim of this article is therefore to set the HXRs in the context of the multi-wavelength flare, to give an up-to-date observational picture, and to provide context for subsequent articles in this volume. This article focuses on the radiative flare, and discusses the coronal mass ejection and solar energetic particles only in association. It is not intended as a comprehensive historical review, although selected historical observations appear. The following recent reviews of observations of flare and related phenomena, including theory, are also recommended for further reading: Priest \& Forbes (2002); Aschwanden (2002); Benz (2008); Krucker et al. (2008a); Vršnak \& Cliver (2008); Schrijver (2009). 

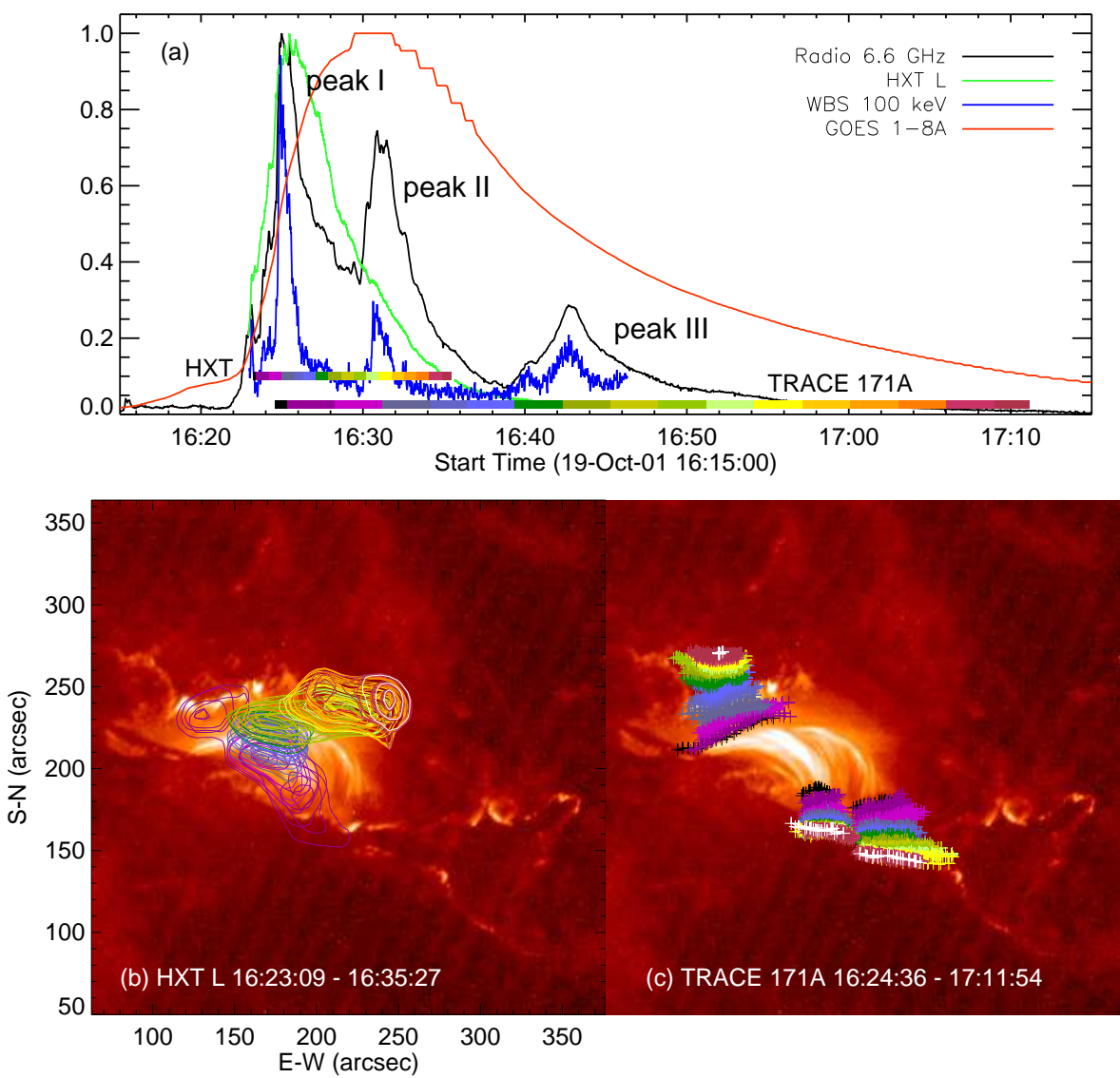

Fig. 1.1 Time evolution of the flare SOL2001-10-19T01:05 (X1.6) in multiple X-ray, EUV, and radio wavelengths (from Qiu et al. 2009). The impulsive phase is best characterized by the hard X-ray light curve (blue, in the $100 \mathrm{keV}$ band of Yohkoh Wide Band Spectrometer (WBS) or the shortest-wavelength radio emissions (black, at $6.6 \mathrm{GHz}$ ), from the Owens Valley Solar Array. The GOES lightcurve (red) shows the gradual phase well. The two panels at the bottom show TRACE $171 \AA$ images defining the flare arcade and its footpoints; left, with hard X-ray contours; right, with EUV footpoint locations color-coded by time in the upper panel. The black line shows the $6.6 \mathrm{GHz}$ microwave emission, at the electron gyrofrequency for a 2,400 G magnetic field.

As a preview of the introductory sections of this article, Figure 1.1 (from Oiu et al. 2009) sketches out the temporal and spatial evolution of a well-observed major flare, SOL200110-19T01:05 (X1.6).

1.1 Flare development

Flares - of all sizes - tend strongly to occur in magnetic active regions and are associated with strong magnetic fields in the neighborhood of magnetic polarity inversion lines (which 
is the dividing line between regions of positive and negative vertical component of the photospheric magnetic field, sometimes also called a neutral line). A small fraction of flares do occur in so-called "spotless" regions (Dodson \& Hedeman 1970; Martin 1980), and largescale filament eruptions with flare-like properties can happen anywhere on the quiet Sun (e.g., Harvey et al. 1986). It is not yet possible to predict the time or location of a solar flare. Several statistical studies have attempted to identify active-region magnetic properties that are correlated with active region flare productivity, or can even act as solar flare forecasters. The best indicators of flare productivity are those known in the "lore" of flare observers regarding the size and complexity of the sunspots in the active region. The largest flares occur in "delta-spot" regions which have two umbrae within a single penumbra (Zirin \& Liggett 1987; McIntosh 1990). They follow the rate of evolution of the active region (Schrijuer et al. 2005) and require the presence of strong magnetic gradients (Hagyard et al. 1984). An example of a flare-productive active region, AR10486, is shown in Figure 1.2 The magnetic properties of flaring regions are encapsulated in various studies of the photospheric field which find higher flare probabilities in regions with high total photospheric magnetic flux, excess magnetic energy, long polarity inversion lines with a strong, highly variable distribution of shear along their length (Cui et al. 2006; Leka \& Barnes 2007) and a high fractal dimension of the photospheric field (McAteer et al. 2005). Incorporating information on the evolution of observed photospheric parameters, the rate of change of the strongest photospheric magnetic twists in the region, is the best predictor of a flare (Leka \& Barnes 2003). However, in general the photospheric properties alone appear to offer poor predictive capabilities, and it appears likely that parameters of the coronal magnetic configuration offer a better prospect. For example, a high degree of complexity - expressed in parameters such as the number of topologically distinct regions (e.g., Cui et al. 2006; Barnes \& Leka 2006), and the "effective connected magnetic field" (Georgoulis \& Rust 2007) - shows promise, as do estimators of the global non-potentiality of the magnetic field such as the flux-normalized field twist (e.g., Falconer et al. 2002). Aside from those methods based on magnetic field information, a Bayesian approach using past history of flare occurrence in an active region has also been proposed (Wheatland 2004).

One final indicator of approaching flare activity that is worth mentioning is filament activation. A filament is a narrow concentration of dense, cool material $\left(n_{e} \approx 10^{12} \mathrm{~cm}^{-3}, T \approx\right.$ $10^{4} \mathrm{~K}$ ) which overlies and runs parallel to a magnetic neutral line. The filament material is supported in the atmosphere by a strongly-sheared magnetic field in the corona, primarily oriented along the neutral line, and it exhibits substantial plasma flows parallel to this. Quiescent filaments are visible in absorption in $\mathrm{H} \alpha$ against the disk (and emission at the limb, against the background sky), and also in absorption in the Transition Region and Coronal Explorer (TRACE) extreme ultraviolet (EUV) lines. Prior to a flare, such features are often observed to start rising slowly. Just prior to their eruption, brightenings in part or all of the filament may also be observed along its length, in wavelengths from $\mathrm{H} \alpha$ to EUV. Filaments and their activation/eruption are interesting in the context of flare development as their involvement in the earliest phase of the eruption, their low altitude in the corona, and their strong concentration along the region of sheared field all point towards the flare initiation, and possibly a large part of the pre-flare energy storage taking place within $1-2 \times 10^{4} \mathrm{~km}$ above the photosphere.

Terminology: Over the decades, the task of describing the appearance and time evolution of a flare led to a flowering of classifications and related vocabulary, which evolved along with our understanding of the range of flare and flare-related phenomena. This included much toing and fro-ing over the direction of causality between flare and CME phenomena (see Cliver 

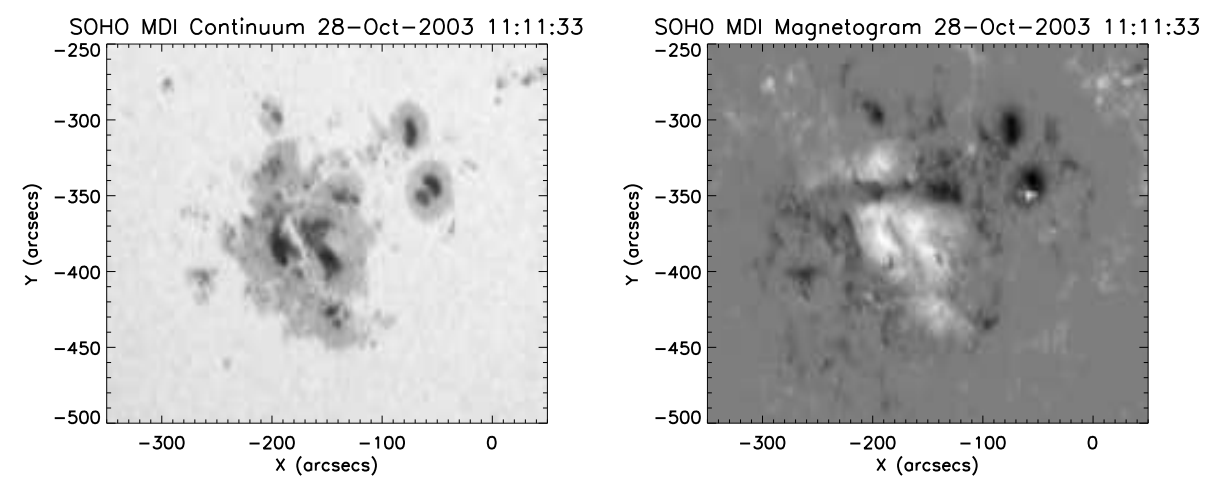

Fig. 1.2 Continuum (left) and magnetogram (right) images of NOAA Active Region 10486, which was the seat of many major flares in late October/early November 2003, including SOL2003-10-29T20:49 (X10.0), SOL2003-11-03T09:55 (X3.9), and SOL2003-11-04T19:53 (X17.4), the most powerful GOES flare on record. The $\delta$ configuration is visible in the positive polarity region of this group, with three major umbrae (those for $\mathrm{X}$ in the range $[-140,-200]$ and $\mathrm{Y}$ in the range $[-370,-410]$ ) within one penumbra. Also visible in the magnetogram are oppositely-colored inclusions within the main spot areas, e.g., at [-140, -390]. These occur as a result of disruption of the magnetically-sensitive spectral line because of the strong disturbance to the atmosphere at the time of the flare. Such features are transient and unrelated to actual magnetic changes. However, non-reversing magnetic changes at the time of flares are observed; these are discussed in Section 3.6

1995, for a historical overview). The time evolution of a flare based on observations is now normally characterized by two main phases, these being the impulsive phase and the gradual phase. The rough division of the time-history of emission into an abrupt and a more slowlyvarying phase has been recognized through the history of flare observations in different wavelengths, including H $\alpha$ (Ellison 1946), microwaves (Wild et al. 1963), and HXRs (Kane 1969; Kane \& Donnelly 1971). "Spotless" flares, mentioned above, tend to have less rapid rises and less significant impulsive phases than flares in normal active regions. Some authors have also classified events as either "gradual flares" or "impulsive flares" based on their time characteristics within a single wavelength range, for example SXRs (Pallavicini et al. 1977) or HXRs (Ohki et al. 1983; Bai 1986) emissions. The notion of the "impulsive flare," i.e., one with no gradual phase has more-or-less faded from parlance among flare physicists, though it persists in discussions of solar energetic particle events. However, exclusively "gradual flares" do occur; such events have essentially no impulsive component and show only a more slowly-varying X-ray component at relatively low peak temperatures - so low, indeed, as to be undetectable by GOES (see Hudson et al. 1995, for example). These are often associated with filament eruptions and have properties similar to "long decay events" (LDEs) that tend to be accompanied by CMEs (Kahler 1977; Sheeley et al. 1983), but which typically do have impulsive phases.

Time evolution: The time evolution of a flare is characterized by different timescales visible at different wavelengths. In the SXR range, following a rise phase lasting a few minutes, evolution is slow. The return of this thermal emission to its pre-event levels is a smooth decay that can last for hours. At the opposite extreme, the lower-frequency end of the radio spectrum exhibits bursts known as type III bursts, with a high brightness temperature, rapid drift rate (frequency decreasing with time) and duration as short as tens of milliseconds (Aschwanden et al. 1995a). These are most normally seen in the decimetric regime and far 
below, but can also be found in the microwaves. Observations of X-rays at tens of keV and above, with the BATSE detector (on the Compton Gamma Ray Observatory, CGRO) exhibit pulses with widths of hundreds of milliseconds to a second or two (see Aschwanden et al. $1995 \mathrm{~b}$, for details). This fast structure is sometimes apparently superposed on a slower, large amplitude variation (though the slow component could be the superposition of many rapid variations). It has not proved possible to detect these short pulses with RHESSI. However with both Yohkoh/HXT and RHESSI, the phenomenon of HXR quasi-periodic pulsations has become very clear (Foullon et al. 2005; Ofman \& Suil 2006; Li \& Gan 2008; Inglis \& Nakariakov 2009). These are modulations in the X-ray intensity, in the few to tens of $\mathrm{keV}$ range with a modulation depth of up to $90 \%$, and observed periods of tens of seconds to a few minutes. The periods observed demand an explanation in terms of MHD timescales; for example, a sausage mode could alter the magnetic field in the loop leading to variations in trapped particle precipitation rates (Inglis \& Nakariakov 2009), or perturbations to the accelerator itself (Ofman \& Sui 2006). Optical flare variations are typically abrupt, tracking the HXR lightcurves well at energies of tens of $\mathrm{keV}$ in the rise phase, but sometimes having a slower decay. UV and EUV also show mixed impulsive and slow variations - major HXR spikes are reflected in the lightcurves, but superposed on a slowly-varying background.

In the HXR impulsive phase, a well-known temporal pattern is the so-called soft-hardsoft spectral pattern - namely that the spectral index of the non-thermal part of the photon spectrum becomes harder as the non-thermal flux increases. This is a pattern that holds universally across individual bursts in the impulsive phase of a flare, both in the footpoint regions and the looptop regions (e.g., Battaglia \& Benz 2006; see also Figure 3.5), and on timescales down to a few seconds. In many flares it also represents the evolution of the entire event at lower time resolution, as the HXR flux increases and then decreases again. However there is a separate class of events - the soft-hard-harder events - in which the spectrum continues to become harder throughout the duration of the event (Silva et al. 2000). This is a property of gradual HXR events (e.g., Cliver et al. 1986; Saldanha et al. 2008), and in at least some events may reflect the presence of long-lived high energy coronal sources (Krucker et al. 2008b). It has been interpreted both as a consequence of energy-dependent particle losses from a coronal trap (with low-energy particles being scattered out of the trap before high-energy particles), and as evidence of continued injection of high energy particles after the flare impulsive phase.

The final time-behavior mentioned here is the Neupert effect - the phenomenon that in many flares the time integral of the non-thermal emissions tracks the time profile of the thermal emissions. The underlying reason for this is that the mostly thermal coronal plasma has a much longer energy loss timescale than the chromosphere, and "integrates" the energy deposited there, presumably from evaporation caused by more impulsive loweratmosphere energy input. This was first discovered as a delay between the peaks of SXR and microwave emissions (Neupert 1968), but has since become more commonly associated with the HXR/SXR time profiles; essentially the HXRs show the (non-thermal) energy release as it happens, and the SXRs show the part of it that winds up in the corona as high-temperature thermal plasma. The fidelity with which the Neupert effect holds depends on the wavelength ranges chosen to test it, with higher temperature thermal emissions showing a better relationship (McTiernan et al. 1999). The Neupert timing relationship was found to hold in $80 \%$ of 66 large events studied using the GOES 1-8 $\AA$ channel and the SMM/HXRBS 26-41 keV channel (Dennis \& Zarro 1993). A statistical study of the timing of the SXR peak compared to the HXR impulsive phase in more than 1000 events, using GOES 1-8 $\AA$ and CGRO/BATSE HXR counts at $25-50$ and $50-100 \mathrm{keV}$, found that $50 \%$ of events were consistent with Neupert-like timing behavior, $25 \%$ were inconsistent, with SXR 
emission peaking substantially after the end of the HXR emission, and the remaining $25 \%$ were unclear Veronig et al. 2002a). In those flares consistent with Neupert timing there was also a strong linear correlation between the HXR fluence (time-integrated counts) and SXR peak flux, as expected. It has been speculated (Li \& Gan 2006) that the flares which do not show the expected Neupert timing behavior may occur if the SXR peak only when plasma "evaporated" from the chromosphere (see Section 3.7) reaches the top of the flaring loops (Reale \& Peres 1995), which introduces a longer time lag for larger flare loops. But the level of disagreement has prompted further investigations of individual flares, in which the beam power inferred from HXR and the power required to explain the SXR flux and spectrum were tested for consistency (Veronig et al. 2005). Under straightforward model assumptions these two powers were found not to correlate well in time, which may suggest that there is energy input other than by non-thermal electrons during some phases of the flare (see counter-examples from Ning 2008). Of course, plasma flows may also lead to heating via compression that might not match the non-thermal signatures so well (e.g. Caspi \& Lin 2010). Violating one particular model assumption, that of a constant value lowenergy cutoff, could (if the cutoff varies through the flare in the right way) lead to better agreement (Sui et al. 2005; Veronig et al. 2005). At the present time, the discrepancies between observed and theoretical Neupert effects are probably within the observational limits, but hints of different physics from the standard ideas that hot thermal emission is coronal and non-thermal emission mostly chromospheric should of course not be ignored.

Cross-correlation analysis has indicated a delay of the SXR time derivative by $\sim 10 \mathrm{~s}$ relative to the HXR flux (Liu et al. 2006). This delay can be interpreted as the hydrodynamic timescale for the redistribution of energy deposited by non-thermal electrons, consistent with the results of radiation hydrodynamic simulations (e.g., Li et al. 1993; Liu 2008; Zharkova et al. 2011).

Preflare evolution: Preceding the impulsive phase, there may be initial signs of activity termed the pre-flare phase. This term covers both pre-flare activity, which refers to the very earliest stages of the flare before the impulsive phase radiation is detectable, and the flare precursor events, which are small-scale brightenings in UV to SXR wavelengths happening some tens of minutes before the flare. Spatially unresolved lightcurve data such as that obtained with GOES may be misleading in this respect, since apparent flare precursors can originate from distant active regions, whereas actual flare precursors may fail to be visible against the integrated intensity from the disk. However with spatially resolved observations it is apparent that flare precursors often do occur in the neighborhood of, but not usually at exactly the same location as, the site in which the majority of the flare radiation will subsequently occur (Fárník et al. 1996; Fárník \& Savy 1998; Warren \& Warshall 2001; Fárník et al. 2003). Some authors have explicitly linked pre-flare brightenings with the destabilization of the magnetic structure that will lead to a CME (e.g., Harrison et al. 1985; Sterling \& Moore 2005) or a filament eruption (Fárník et al. 2003; Chifor et al. 2007; Liu et al. 2009d) although coronagraphic studies of the former kind are plagued by the lack of knowledge of the CME launch time, since substantial parts of the event occur unseen below the coronagraph occulting disk. The onset of pre-flare activity, in the form of weak SXR emission, precedes the onset of impulsive HXR emission by around 3 minutes in the vast majority of flares, regardless of their total energy or duration (Veronig et al. 2002b). Spectral line broadening has been observed in the pre-flare phase (Harra et al. 2001, 2009) starting minutes to hours before the impulsive phase, consistent with non-thermal effects such as plasma turbulence. 
1.2 The impulsive phase

The primary energy release occurs during the impulsive phase. This phase of flare activity lasts from tens of seconds to tens of minutes and is characterized by HXRs, $\gamma$-ray, nonthermal (synchrotron) microwaves and white-light continuum emission, indicating strong acceleration of both electrons and ions. These radiations are accompanied also by strong enhancements in chromospheric line and continuum emission, ultraviolet and extreme ultraviolet radiation, and bulk plasma upflows in the EUV and SXRs at speeds on the order of $100 \mathrm{~km} \mathrm{~s}^{-1}$ coupled with downflows in cooler lines such as $\mathrm{H} \alpha$ (Zarro et al. 1988; Milligan et al. 2006a). The impulsive-phase radiation is concentrated at the chromospheric endpoints of the magnetic field involved in the flare; indeed prior to the availability of the EUV and SXR imaging that has led to a shift of focus to the coronal aspects of flares, the strong lower atmosphere signatures led to the term "chromospheric eruption" or "chromospheric flare" being used in early studies (Giovanelli 1948) even as the relationship of the radiation increase to flows and to processes higher in the atmosphere and at the Earth were becoming apparent. Even now, it is clear that while the magnetic drama takes place in the corona, the dominant radiative energy of a flare, from both non-thermal and thermal particles, is from the lower atmosphere (e.g., Chupp et al. 1973; Hudson 1972; Kane et al. 1979b; Lemaire et al. 2004; McIntosh \& Donnelly 1972; Woodgate et al. 1983). So somehow the flare energy must be transported into the chromosphere, there to be dissipated by radiation and flows. There is unfortunately only very sparse knowledge of the optical and UV properties of the impulsive phase, and this is a substantial shortcoming and missed opportunity. Rich diagnostic information, particularly in spectroscopic data, informs our understanding of the quiet-Sun chromosphere, and to have such data available also for flaring regions would substantially improve our knowledge of the results of flare energy deposition in these layers, and even give insights into the flare particle acceleration problem.

The magnetic reconfiguration that allows the rapid release of stored magnetic energy in a flare is generally agreed to occur somewhere above the chromosphere in the (low- $\beta$ ) corona. The main theoretical argument for a coronal energy release is that the corona provides adequate volume for storing the energy required for a flare. Observationally, the coronal manifestations such as large SXR and H $\alpha$ flare loops, HXR looptop sources, and coronal mass ejections have almost universally been interpreted in a framework involving large-scale coronal magnetic reconnection. Non-linear force-free reconstructions of the magnetic field find that the energy is concentrated low in the corona in a newly-emerged active region, and can be sufficient for flaring activity (Régnier \& Priest 2007; Schrijver et al. 2008) whereas in an older decaying active region it is stored higher in the corona. It has proved marginally possible to detect differences between the free magnetic energy before and after a flare or CME event (Metcalf et al. 2002; Blevbel et al. 2002; Schrijver et al. 2008). More quantitative reconstructions of the coronal magnetic field which can track the actual redistribution of coronal magnetic energy during a flare are just beginning to appear (Jing et al. 2008; Thalmann \& Wiegelmann 2008).

The impulsive-phase flare signatures in the lower solar atmosphere are termed "footpoints" (originally HXR) or "ribbons" (originally $\mathrm{H} \alpha$ ) and are now detectable in a wide range of wavelengths. They are interpreted as the chromospheric ends of the coronal magnetic field structures involved with the flare energization at a given instant. The impulsivephase reconnection of the coronal magnetic field is not visible in coronal signatures with current instruments, though its effects certainly may be. These effects include the EUV and SXR flare loops and cusp-shaped structures that appear in the gradual phase (see Section 1.3) and impulsive phase supra-arcade downflows (Asai et al. 2004b; Khan et al.2007), 


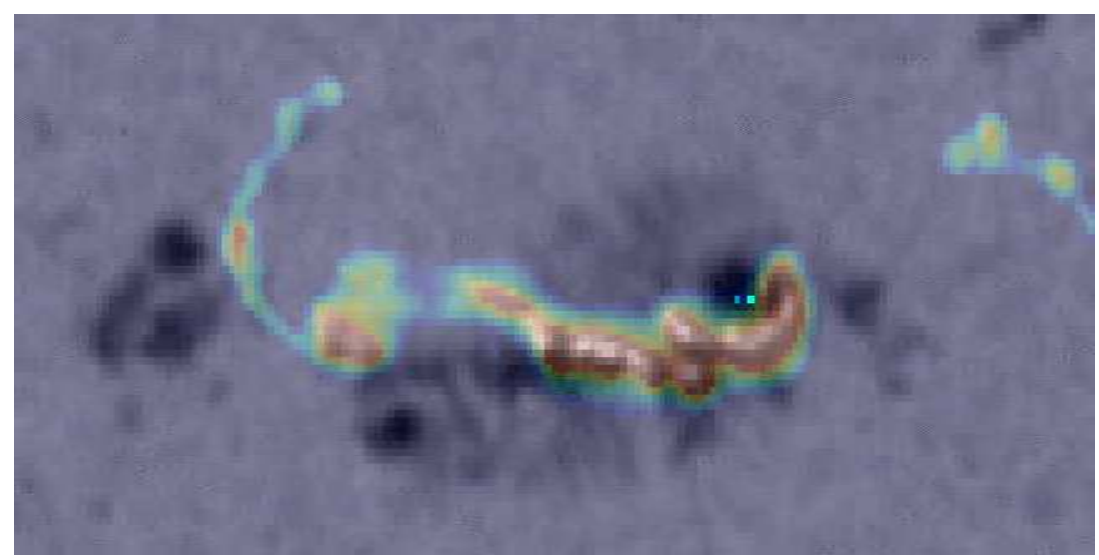

Fig. 1.3 SOL2002-10-04T05:38 (M4.0), with TRACE UV (1700 ̊) ribbons in color overlaid on TRACE continuum in black and white. The flare white-light sources are visible as small white patches, within the UV ribbons, and having a considerably smaller area. The field of view is $135^{\prime \prime}$ by $65^{\prime \prime}$, pixel size $0.5^{\prime \prime}$. Here the main neutral line lies between the concave-up UV feature under the sunspot on the right, and the more linear feature below it.

interpreted as the "dipolarization" of newly-reconnected field. These correspond extremely well in time with impulsive phase HXR bursts. Flare footpoints occur on either side of the magnetic polarity inversion line, as illustrated in Figure 1.3 Early in the impulsive phase they tend to be concentrated around this line, and move with respect to it as the flare evolves. In the later phase of a flare, when strong $\mathrm{H} \alpha$ and UV ribbons are visible, the ribbons tend to move outward from the polarity inversion line, but in the impulsive phase, both ribbon and HXR footpoint motion is more complex, sometimes showing parallel or approaching motions. This is discussed in more detail in Section 3.4

The importance of flare ribbons and footpoints in marking regions of changing magnetic connectivity is now well established (e.g., Mandrini et al. 1991). The large-scale reconnection model in two dimensions (e.g., Kopp \& Pneuman 1976) was originally developed to explain the spreading $\mathrm{H} \alpha$ ribbons and the $\mathrm{H} \alpha$ arcade that appears in the gradual phase of a flare. The outer edges of the ribbons show the chromospheric projection of the interface (magnetic separatrix surface) between the post-reconnection ("post-flare") arcade fields, and the field that is yet to be reconnected. The importance to flare energy release of the threedimensional connectivity of the solar magnetic field around sunspots was known early on (Giovanelli 1948; Sweet 1969; Syrovatskii 1981) and early observational associations were made between topological structures and observable chromospheric $\mathrm{H} \alpha$ features by e.g., Gorbachev \& Somov (1989), Mandrini et al. (1991), and Demoulin et al. (1992). Interpreting the chromospheric features within this framework, it becomes possible to establish some of the global properties of the magnetic field and its evolution, such as the different magnetic domains, the amount and rate of magnetic flux transfer during flare events, and also under the assumption of two-dimensional translational symmetry - the convective electric field $(\mathbf{v} \times \mathbf{B})$ of the magnetic reconnection. 

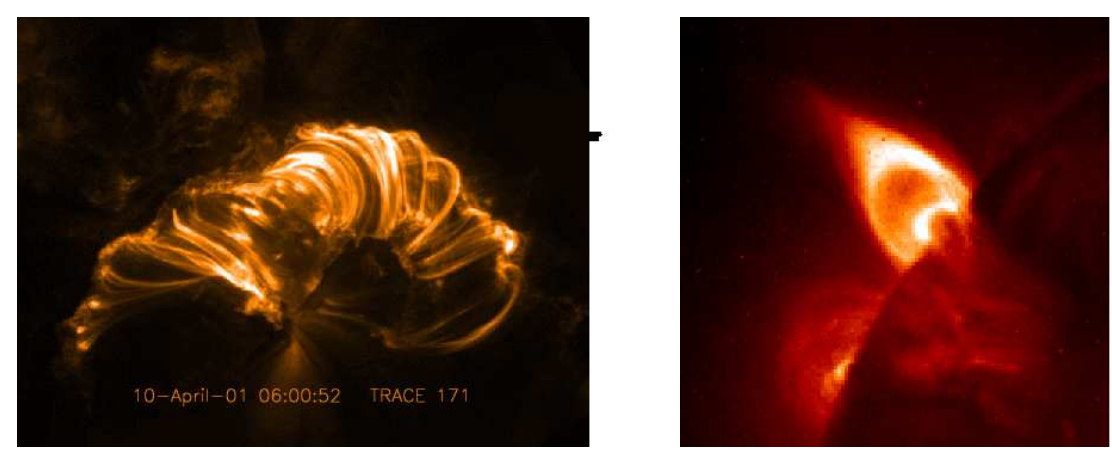

Fig. 1.4 Left: a beautiful flare arcade (SOL2001-04-10T05:26, X2.3) seen here in the $171 \AA$ channel of TRACE, revealing plasma at $\sim 1 \mathrm{MK}$ emitting in lines of Fe IX/X. Right: a large post-flare cusp structure observed several hours after the impulsive peak of SOL1999-03-18T08:31 (M3.3) by Yohkoh/SXT and reported by Yokovama et al. (2001). The temperature in this structure is 3-4 MK.

\subsection{The gradual phase}

During the gradual phase, identified by its slowly decaying SXR and microwave signatures, the effects of the flare on the corona become apparent. Loops and loop arcades emitting in SXRs and EUV form and appear to grow (Figure 1.4), filled (it is usually assumed) by chromospheric plasma forced to expand into the corona as the chromosphere is rapidly heated by particle energy deposition, or by thermal conduction. This expansion is known as chromospheric evaporation (see Section 3.7). The gas pressure of these flaring coronal loops can increase from $\sim 0.1$ dyne $\mathrm{cm}^{-2}$, typical of the quiet corona, to $>10^{3}$ dyne $\mathrm{cm}^{-2}$, as shown by semi-empirical models based on radiative transfer theory in chromospheric lines (e.g., Machado et al. 1980). The pressure increase is mainly due to the growth of density in the loops, but the new coronal material is also at flare temperatures (10-20 MK), as opposed to pre-flare coronal temperatures (1-3 MK). The loop arcades show a gradient in temperature, with the outermost loops (i.e., those corresponding to the outer edges of the ribbons) being the hottest (Forbes \& Acton 1996). The hottest outer loops sometimes exhibit a "cusp" (Figure 1.4 consistent with the shape of the field that would be expected below a coronal current sheet; this cusp is most pronounced later in the gradual phase of the flare (Tsuneta et al. 1992; Yokoyama et al. 2001; Hara et al. 2008). This is circumstantial evidence for coronal magnetic reconnection during this phase. Later on, as the corona cools, the arcade becomes visible at lower temperatures including EUV and $\mathrm{H} \alpha$ (Schmieder et al. 1995). Cooling occurs by both conduction and radiation, depending on the flare loop length and plasma parameters (Culhane et al. 1970; Cargill et al. 1995; Aschwanden \& Alexander 2001). Observational studies tend to find that early on the hottest plasma cools for a few minutes by conduction (e.g., Culhane et al. 1994) which may then be followed by dominant radiative cooling (e.g., Aschwanden \& Alexander 2001; Vršnak et al. 2006). Models have been formulated which also take into account the thermal energy redistribution throughout the loop due to conduction, and the gentle chromospheric evaporation that results Cargill et al. 1995). As the loop plasma cools it begins to drain under gravity, and $\mathrm{H} \alpha$ downflows ("coronal rain") become visible along the legs of the arcade. The plasma upflows and downflows have been detected spectroscopically in a number of events (e.g., Zarro \& Lemen 1988; Czaykowska et al. 1999; Brosius 2003). 


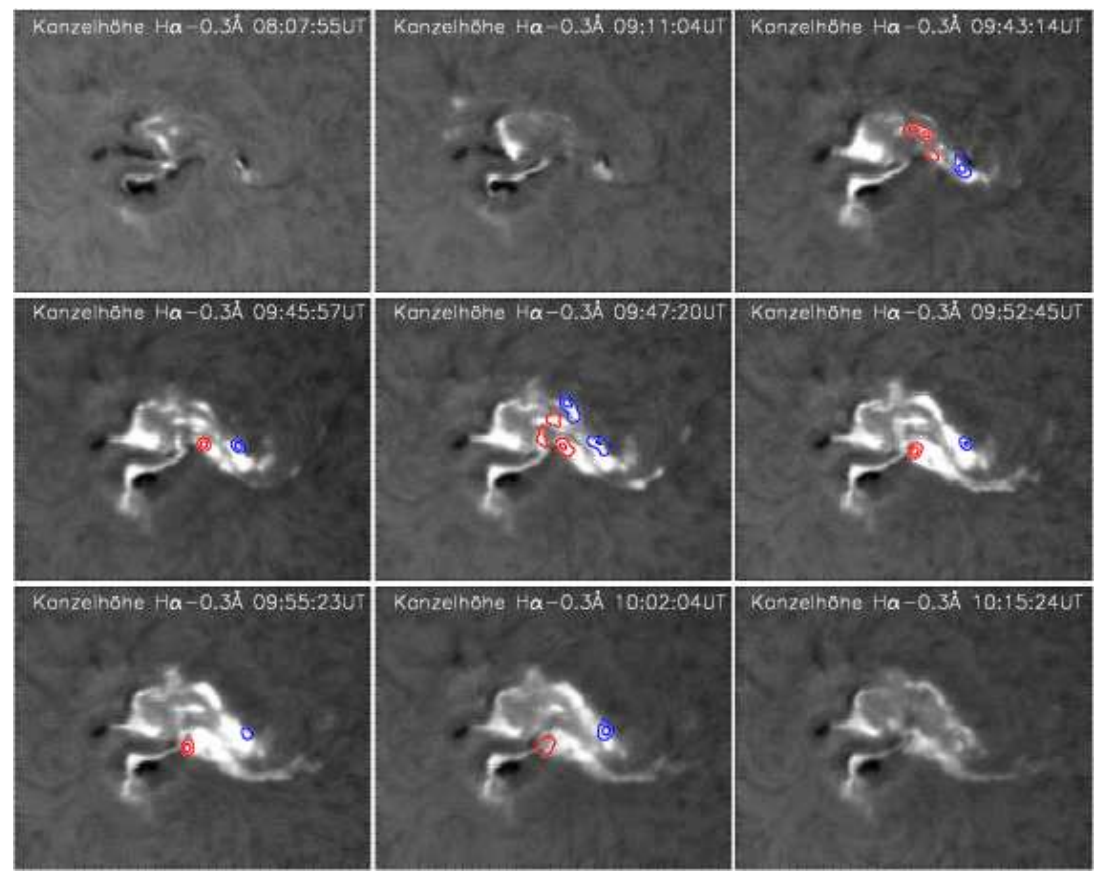

Fig. 1.5 Sequence of $\mathrm{H} \alpha-0.3 \AA$ filtergrams taken at Kanzelhöhe Observatory during SOL2005-01-17T09:52 (X3.8) in a $400^{\prime \prime} \times 300^{\prime \prime}$ field of view. The contours are co-temporal RHESSI 30-100 keV images, and the color indicates the polarity of the underlying photospheric magnetic field (red: positive, blue: negative). Before 09:35 UT, no RHESSI data were available. During certain flare periods, up to five individual HXR footpoint sources were observed simultaneously, located on different $\mathrm{H} \alpha$ flare ribbons, but two main footpoints prevailed during the overall flare impulsive phase. Note the general counterclockwise rotation of the line connecting the opposite-polarity footpoints, an observation often interpreted as a reduction in shear. Adapted from Temmer et al. (2007).

Loop arcades observed at a particular wavelength appear to grow upwards and outwards in time. This can be seen in $\mathrm{H} \alpha$ (Švestka et al. 1987), microwaves (Li \& Gan 2005), EUV, SXRs and even HXRs (e.g., Gallagher et al. 2002). In the common interpretation, the point of magnetic reconnection moves slowly upwards in the solar corona as the gradual phase proceeds. Successive shells of reconnected loops fill with hot plasma expanding from the chromospheric footpoints, a pattern often described as the "CSHKP model" after some of its major contributors (H. Carmichael, P. Sturrock, T. Hirayama, R. Kopp, and J. Pneuman). As new loops or their footpoints brighten in a particular wavelength, their angle with respect to the magnetic polarity inversion line may increase, as reported since Skylab in many events (e.g., Sakurai et al. 1992; Sakao 1994; Masuda et al. 2001; Aschwanden \& Alexander 2001; Su et al. 2006; Schmahl et al. 2006; Ji et al. 2008; Liu et al. 2009b). This could be interpreted as due to reconnection starting in highly-sheared field anchored close to the polarity inversion line, and progressing to less-sheared field further from it. Figure 1.5 illustrates this process.

The gradual phase may last several hours, depending on the magnitude of the flare. In many events, the cooling timescales point towards an additional energy source during the gradual phase, which could come from ongoing slow reconnection and its associated heating (MacCombie \& Rust 1979; Forbes et al. 1989), either above the flare arcade or conceivably 
also between individual tangled strands of the arcade as they shrink down. The arcade appears to grow in scale as newer and higher-altitude loops appear, while individual loops physically shrink with time (Švestka et al. 1982; Forbes \& Acton 1996). Multi-strand loop modeling (Hori et al. 1998; Warren et al. 2002; Reeves \& Warren 2002) may explain cooling time profiles, and also the fact that the observed velocity characteristics of evaporative upflows tend to be smaller than those predicted by radiation hydrodynamic simulations.

The gradual phase and the standard magnetic-reconnection model nicely link the arcade formation with the occurrence of a filament eruption and a CME; this would create the (approximately) oppositely-directed field lines that then reconnect (Section 5). Further evidence for ongoing magnetic coronal reconnection comes from the supra-arcade downflows (McKenzie \& Hudson 1999; Sheeley et al. 2004) observed mainly during the gradual phase and apparently moving at a fraction of the Alfvén speed. As we have seen already, such downflows also occur in the impulsive phase of a flare. The magnetic geometry in which the reconnection and shrinkage occur may be considerably more complex in the impulsive phase.

\subsection{The magnetic field}

How well do we understand the structure of the magnetic field, which underlies all aspects of solar activity? There are routine measurements of the Zeeman splitting in the solar photosphere (see Section 2.3), especially for the line-of-sight component of the field. We can identify the magnetic environment of flare occurrence morphologically - a large, rapidlyforming sunspot group containing a "delta spot" certainly has a higher probability of flaring (e.g., Zirin \& Liggett 1987). At the photospheric level, the polarity inversion line ("PIL" or "neutral line") plays an important role morphologically. This line by definition must separate the footpoints of coronal loops, such as flare loops. The field, again by definition, lies tangent to the plane of the photosphere at the neutral line, and where it is concave up (the "bald patch" configuration) it does not lead to an overlying arcade, but still may play an important role in flare development (Titov et al. 1993).

Unfortunately the field in the photosphere, which can be observed comprehensively, only maps into the corona in an elusive manner (e.g., Schrijver et al. 2008). Many competing mathematical and MHD-based approaches have been applied to the problem of extrapolating the photospheric knowledge into the corona (Schrijver et al. 2006), but several substantial problems prevent these techniques from being quantitatively persuasive. From the perspective of flare research, one major objective (for example) would be to characterize the "non-potential energy" resulting from current systems linking the corona and the solar interior. This energy exceeds the minimum level derivable from a "potential field" representation, i.e., one without embedded currents. This should decrease when a flare happens, but the results of all analyses to date have been uncertain on this point (e.g., Metcalf et al. 2002).

The direct measurement of the field via line-splitting observations in an active-region corona has met extreme difficulties, and accordingly the results at the present time have great uncertainties. In principle one needs to measure a full vector function $\mathbf{B}(\mathbf{r})$ at each point $\mathbf{r}$ in the coronal volume. There is progress on this formidable task (e.g., Tomczyk et al. 2008). Radio techniques also have great promise to determine at least $|\mathbf{B}|$, though, since the Larmor frequency and its harmonics have clearly identifiable and precise signatures (e.g., Bastian et al. 1998). In general the most exact knowledge of at least the plane-of-thesky projection of $\mathbf{B}(\mathbf{r})$ comes from high-resolution imaging in coronal emissions (visible, 
UV, EUV, X-ray), since they often have fine striations that can be interpreted as due to the alignment of the field. A potential-field interpretation of the intensity in the corona above a large sunspot can be approximated by that of a simple current loop lying in the photosphere: $B(z) / B_{0}=\left(z^{2}+1\right)^{-3 / 2}$, where $\mathrm{z}$ is the height above the umbra in units of the umbral radius. For a radius of $10^{4} \mathrm{~km}$ and an umbral field of $3500 \mathrm{G}$ (e.g., Cox 2000) this formula gives axial fields of $300-1200 \mathrm{G}$ at coronal altitudes of $1-2 \times 10^{4} \mathrm{~km}$. For reference, the current in the loop has a magnitude of $5.5 \times 10^{12} \mathrm{~A}$.

When a solar flare happens, the observed photospheric field changes in a stepwise manner (Wang 1992; Sudol \& Harvey 2005). This would be expected from the extraction of energy by its dipolarization following large-scale reconnection, or generally just to reduce $\int\left(B^{2} / 8 \pi\right) d V$ (Hudson 2000); see Section 3.6

\section{Status of observational techniques}

Flare emissions have been detected all the way from about $10^{-10} \mathrm{eV}(30 \mathrm{kHz}$, a typical plasma frequency in the solar wind near the Earth) out to some hundreds of $\mathrm{MeV}$ (the pion decay spectrum). This whole vast spectrum, in principle, could be broadly observed with sensitive remote-sensing instruments, and in stereo. We could thus aspire to the observation of a many-dimensional data cube: $\mathrm{x}, \mathrm{y}, \mathrm{z}, \lambda$, polarization, and time for starters, and even the directional components (limb-darkening function) of the emitted radiation. Clearly the observations to date have only begun to scratch the surface of this potential wealth of material. In each parameter there is an implied sampling capability - temporal cadence, signal-tonoise ratio, contrast, and scattered light considerations provide much further diversity. The limits on the available capabilities of course relate to somewhat intangible matters such as community preferences, technical feasibility, and cost. We note that solar observers tend (for several reasons) to want to study the most powerful events, whereas non-solar astronomers may strive for sensitivity instead. Unfortunately, this leaves a huge range of solar parameter space unobserved by the best instrumentation. In the following we briefly review the capabilities of the available multiwavelength observations existing at present, broken down by wavelength.

\subsection{Radio}

The radio observations broadly reveal both non-thermal and thermal emissions via several mechanisms, and from a broad range of phenomena occurring anywhere above the photosphere (e.g., Bastian et al. 1998). For flares the most important of these are the cm-wave gyrosynchrotron radiation, for high-energy electrons (the extension, to a few $\mathrm{MeV}$, of the energetically important electrons responsible for the impulsive-phase HXR emissions), and the meter-wave plasma-frequency emissions that show many dynamical processes in the corona.

The past two decades have seen the improvement and deployment of several instruments. The major dedicated $\mathrm{cm} / \mathrm{mm}$-wave observatory is the radioheliograph at Nobeyama, Japan (e.g., Koshiishi et al. 1994) which images at fixed frequencies of 17 and $34 \mathrm{GHz}$ with a beam size at $17 \mathrm{GHz}$ of $10^{\prime \prime}$. This is accompanied by a non-imaging radio polarimeter operating at nine fixed frequencies between 1 and $80 \mathrm{GHz}$. The Owens Valley Solar Array (OVSA) (Hurford et al. 1984; Gary \& Hurford 1999) has a smaller number of dishes but 
superior frequency coverage, operating at 86 fixed frequencies from 1 to $18 \mathrm{GHz}$. This allows the use of "frequency synthesis" (i.e., the interpretation of the measured (u,v)-plane 1 in terms of a model source spectrum) to augment the coverage in the (u,v)-plane to a certain extent.

At decimeter wavelengths the most productive facility is the Nançay radioheliograph (e.g., Avignon et al. 1989), typically operating at five fixed frequencies in the $150-450 \mathrm{MHz}$ range. Joint RHESSI-radio observations have also been made with a number of dynamic spectrographs spanning a wide range of frequencies, such as Ondrejov at $0.1-4.2 \mathrm{GHz}$ (Jiricka et al. 1993), Huairou at 2.6-3.8 GHz (Fu et al. 2004), the Phoenix-2 instrument at 0.1-4 GHz (Messmer et al. 1999), Tremsdorf at 40-800 MHz (Mann et al. 1992), the Greenbank Solar Radio Burst Spectrometer at 10-110 MHz (White et al. 2005a) and Hiraiso at 25-2500 MHz (Kondo et al. 1994). There are plans for an ambitious new solar radio interferometer, the Frequency Agile Solar Radio Telescope (FASR; Bastian 2003) to cover the range 0.1 to $24 \mathrm{GHz}$, while low-frequency solar imaging and spectroscopy is planned with the Low Frequency Array for Radio Astronomy (LOFAR, Bastian 2004); see Claßen et al. (2003) and Benz et al. (2005). At submillimeter wavelengths a dedicated site now exists at El Leoncito, Argentina, where the Solar Submillimeter Telescope observes at frequencies of a few hundred GHz (Kaufmann 2003).

\subsection{Infrared}

The solar infrared spectrum extends from visible wavelengths out to the $10 \mu \mathrm{m}$ band accessible to ground-based observations, the mid-infrared accessible only from space, and the submm-THz range again accessible from the ground. This huge region contains thermal free-free continuum and coronal line emission, plus other possible contributors at the longest wavelengths. The IR has some advantages, for example, in terms of improved seeing and in magnetic sensitivity (e.g., Debi Prasad 1998); on the other hand the diffraction limit becomes severe for non-interferometric imagers.

No dedicated solar infrared observatories at wavelengths much longward of $1 \mu \mathrm{m}$ exist at present although developments are underway in imaging at $10 \mu \mathrm{m}$ (e.g., Hudson 1975), with flares already having been detected (Melo et al. 2006). Specific observations on the $1 \mathrm{~m}$ McMath Solar Telescope make use of the mid-infrared to study photospheric magnetic fields (Moran et al. 2007), and at the $0.76 \mathrm{~m}$ Dunn Solar Telescope to probe flare activity at the "opacity minimum" spectral region around $1.5 \mu \mathrm{m}$ (Xu et al. 2004). Direct observations of the coronal magnetic field are also becoming possible (Lin et al. 2004; Tomczyk et al. 2008). Full disk observations are made daily in the Ca II $8542 \AA$ and He I $10830 \AA$ lines by the SOLIS synoptic telescope (and by earlier Kitt Peak telescopes), and flare spectroscopic observations at these wavelengths are provided by the Multichannel Infrared Solar Spectrograph (MISS; Li et al. 2002) at Purple Mountain Solar Observatory (PMO), with a spectral resolution on the order of $1 \AA$.

\footnotetext{
1 The radio astronomers" "(u,v)-plane" is the map of observed spatial frequencies; Fourier transformation of measurements at the observed points in the $(\mathrm{u}, \mathrm{v})$-plane yields the source image projected on the plane of the sky.
} 
2.3 Optical

General: High-resolution observations have become much more commonplace from groundbased observatories. This results both from the availability of large apertures but especially from active control of the optics to ameliorate the effects of atmospheric seeing; in addition there are advanced post-processing techniques such as speckle reconstruction (von der Luehe 1993). Specific telescopes include the Dunn Solar Telescope, the Swedish Solar Observatory on La Palma, the German Vacuum Tower Telescope on Tenerife, and the Dutch Open Telescope. The best of these observations have substantially better spatial resolution than contemporaneous telescopes in space, but without the consistently perfect seeing conditions.

Several flexible optical/IR instruments operate in user mode at ground-based observatories (e.g., Cauzzi et al. 2008). With such instruments one can generate multidimensional data cubes incorporating spatial, temporal, spectral, and polarimetric measurements. Being ground-based observatories, they may operate with state-of-the-art adaptive optics and, having large apertures, obtain high resolution while running at high cadence. They also have minimal constraints on data bandwidth and can flexibly study a variety of spectral features. Future instrumentation is emphasizing larger apertures, higher frame rates (Jess et al. 2007), and the implementation of high-order adaptive optics (e.g., Rimmele et al. 2003; Denker et al. 2007). Major new facilities under study include the Advanced Technology Solar Telescope (ATST) and the European Solar Telescope (EST).

Optical observations from space have included the white-light telescope on Yohkoh, which despite being short-lived, observed several solar flares in the Fraunhofer G band (Matthews et al.2003). In the RHESSI era, the Michelson Doppler Imager instrument on the Solar and Heliospheric Observatory (SOHO) (MDI; Scherrer et al. 1995) provides whitelight full disk images, and the TRACE satellite also has a "white-light" channel with a broad response $(\sim 1000-8000 \AA)$ which was successfully used in the study of solar flares at high cadence (Hudson et al. 2006). However, the first full large-aperture optical solar observatory in space, on board Hinode (Shimizu 2002), was only launched in 2006. This telescope provides diffraction-limited observations with a $50 \mathrm{~cm}$ primary and includes both narrowband filter imaging and spectrographic imaging, the latter permitting vector magnetograms (e.g., Lites et al. 2001).

$\mathbf{H} \alpha$ : The $3 \rightarrow 2$ Balmer transition line of neutral hydrogen, centered at $6563 \AA$, is historically important in flare observations and continues to be observed and studied for its rich diagnostic capability. Many tens of observatories worldwide are engaged in monitoring the Sun in $\mathrm{H} \alpha$ for flares, making both full- and partial-disk observations. The US National Oceanic and Atmospheric Administration (NOAA) maintains a database of $\mathrm{H} \alpha$ flares stretching back decades. Currently five stations provide $\mathrm{H} \alpha$ data for this: Kanzelhöhe (Austria), Learmonth (Australia), Holloman Air Force Base (USA), San Vito (Italy) and Kharkov (Ukraine). The major $\mathrm{H} \alpha$ instruments with facility for high cadence and high resolution observations, which have been used in connection with RHESSI observations, are at Kanzelhöhe (Otruba \& Pötzi 2003 ), the $65 \mathrm{~cm}$ telescope at Big Bear Solar Observatory (removed from service in 2006, and being replaced with a $1.6 \mathrm{~m}$ instrument), and the $\mathrm{H} \alpha$ Fine Structure Telescope at Purple Mountain Observatory. The Global High-Resolution H-alpha Network consists of the stations at Big Bear, Kanzelhöhe, Catania, Meudon, Pic du Midi, Huairou, Yunnan, and Mauna Loa. Spectroheliographs making $\mathrm{H} \alpha$ observations (other than daily images) include those on the THEMIS telescope (Mein 2002) and at Hida Observatory. 
Magnetographic: It is difficult to measure the magnetic field in the solar corona directly. Photospheric glare makes observations against the disk almost inconceivable except perhaps at radio wavelengths; above the limb one has the high temperatures of the corona and lineof-sight confusion to contend with. The photospheric magnetic fields are typically observed in a weak Fraunhofer line, Ni I $6758 \AA$ for MDI.

Nevertheless, precise knowledge of the vector magnetic field in the corona is fundamental to our understanding of flares and CMEs, and much effort is expended in extrapolating near-photospheric measurements into the corona. To understand the coronal field, the measurements would ideally be done above the solar chromosphere where the magnetic field approaches the force-free condition and can therefore be used as a reliable lower boundary for mathematical extrapolations into the corona, if it remains static. Special observational facilities exist for the observation of the solar magnetic field in the photosphere via spectropolarimetric techniques, with perhaps the earliest serious work done at the Crimean Observatory (Severny 1964). These facilities have different objectives, ranging from characterization of "sun-as-a-star" average line-of-sight field components (e.g., Wilcox \& Hundhausen 1983), to full vector measurements at the highest possible resolution and at different heights in the solar atmosphere. The latter data provide the indispensable boundary condition for the increasingly ambitious efforts to extrapolate the structure of the coronal field in three dimensions (e.g., Schrijver et al. 2006). Ground-based magnetographs include the early MSFC Vector Magnetograph (Hagyard et al. 1982), the Mees Imaging Vector Magnetograph (Mickey et al. 1996), Huairou (Ai \& Hu 1986), the Hida Solar Magnetic Activity Research Telescope (SMART; Ueno et al. 2004), the BBSO Digital Vector Magnetograph (Spirock et al. 2001), the GONG Network, including magnetographs located in 6 sites around the world (Harvey et al. 1996), and SOLIS (Keller et al.2003). The Hinode spectropolarimeter, which uses the Fe I lines at 6302.1 and $6302.5 \AA$, is the only high-resolution vector magnetograph in space, while the HMI instrument on the Solar Dynamics Observatory now provides high-cadence, full-disk photospheric vector magnetograms at a $1^{\prime \prime}$ spatial resolution (Graham et al. 2003).

The coronal magnetic field, in spite of frequent small-scale activity, normally is considered to be force-free. Thus it contains currents, so that a simple extrapolation of any photospheric magnetic field, the (non-force-free) source of the stresses that drive these currents, may incorporate systematic errors. Accordingly several observatories have begun systematic observations of the chromospheric magnetic field in Na I $5896 \AA$ (Metcalf et al. 1995) or another chromospheric line such as $\mathrm{H} \beta$. The upper chromosphere should be more force-free and these observations should enable more accurate extrapolations.

\subsection{UV-EUV-X-ray wavelengths}

From the UV into the SXR domain (up to a few $\mathrm{keV}$ ) 2 one can use specialized astronomical techniques involving normal-incidence or, at the shortest wavelengths, grazing-incidence mirrors. Such observations need to be made in space because of the large opacity of the Earth's atmosphere. Indeed, solar observations at these previously inaccessible wavelengths were a part of the early history of space astronomy, leading to Skylab. The following paragraphs summarize some of the observations concurrent with RHESSI.

2 Roughly, the UV and vacuum UV extend through the Lyman continuum; at shorter wavelengths to about $44 \AA$, the carbon K-edge, it would be EUV, and shortward still soft X-rays. 
Ultraviolet: For spectroscopy, SUMER (Wilhelm et al. 1995) has provided excellent data but with limited solar flare coverage for technical reasons. TRACE has provided excellent imaging observations but with poor spectral resolution (Handy et al. 1999). The TRACE UV bands includes a narrow $(\sim 30 \AA)$ band centered on strong lines of $\mathrm{C}$ IV, plus broader UV channels covering Lyman- $\alpha$ and the UV continuum to around $2500 \AA$, and a "white light" channel covering the full range of spectral response of the Lumogen-coated chargecoupled device (CCD 4 . Nevertheless this rich spectral domain, the most important for studying the chromosphere and transition region, still remains underexploited; for example, there are no comprehensive observations yet even of Lyman- $\alpha$, the strongest line.

EUV: Again TRACE has provided excellent EUV observations at the standard normalincidence narrow spectral bands centered at 171,195 , and $284 \AA$, corresponding to transitions of Fe IX/X, Fe XII (and Fe XXIV in flares), and Fe XV. Such observations, pioneered by the EIT 5 instrument (Delaboudinière et al. 1995), now continue stereoscopically (Kaiser et al. 2007) on the STEREO spacecraft (Howard et al. 2008). In addition to these broad-band imaging instruments, stigmatic slit spectrographs have flown on SOHO (310-

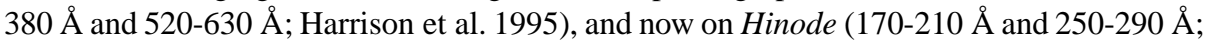
Culhane et al. 2007). These provide high resolution spectroscopy enabling detailed diagnostics for plasma density, temperature, velocity and abundance. Currently the AIA 6 instrument (Rochus et al. 2004) on SDO provides comprehensive coverage of this type, with $10 \mathrm{~s} \mathrm{ca-}$ dence, many passbands, and whole-Sun imaging with negligible gaps in time coverage.

Soft X-rays: The SXR flux from a solar flare has become the definitive flare observable via the GOES flare classifications, and photometric SXR observations have continued in an unbroken photometric sequence since the 1970s. High-resolution imaging began with grazingincidence telescopes on rockets and then (with film readout) on Skylab. In 1991 Yohkoh was launched with its SXT imager (Tsuneta et al. 1991) which could image at spatial resolutions down to $5^{\prime \prime}$, and now Hinode carries the improved XRT instrument (Golub et al. 2007). The temperature coverage of XRT is around 1-30 MK, and multiple filter combinations permit a temperature discrimination as good as $\log \mathrm{T}_{\mathrm{e}}=0.2$.

In terms of energy, the end of the soft X-ray range and the beginning of the hard X-ray range is a somewhat arbitrary matter, which varies from author to author in solar physics, and also varies from field to field in astrophysics. Roughly speaking, energies of $0.1-10 \mathrm{keV}$ are counted as "soft" X-rays in solar physics, and energies above about $20 \mathrm{keV}$ are "hard." Between these two, the description "soft" or "hard" can depend on whether the spectrum looks thermal or non-thermal.

Spectroscopy: High-resolution spectroscopy (from infrared to the $\gamma$-rays) provides the closest approach to learning the state of the flaring plasma via remote sensing. Generally these techniques provide information about the electron energy distribution function, but extracting information about the electron angular distribution is extremely difficult, even adopting the common assumption of azimuthal isotropy with respect to the magnetic field. The SXR emission lines have been particularly productive in flare research, with instruments flown on many spacecraft - most recently the Yohkoh Bent Crystal Spectrometer (Lang et al. 1992)

\footnotetext{
3 The Solar UV Measurements of Emitted Radiation instrument on SOHO.

4 Lumogen is a proprietary coating that extends the UV response of the CCD.

5 Extreme-EUV Imaging Telescope on $\mathrm{SOHO}$.

6 Atmospheric Imaging Assembly.
} 
and its follow-on RESIK 7 (Sylwester et al. 2005). The latter observes in narrow bands around the principal X-ray emission lines of $\mathrm{Si}, \mathrm{S}, \mathrm{Cl}, \mathrm{Ar}$, and $\mathrm{K}$ in the 3.1-6.6 $\AA$ range, with access to other features at higher grating orders.

At longer wavelengths the definitive observations have come from stigmatic slit spectrographs, which image in one dimension and thus multiplex the second spatial dimension in time. The definitive instruments in this category are not particularly optimized for flare observations but have produced many interesting results. These are currently SUMER (Wilhelm et al. 1995), which covers the VUV range 500-1610 A, and EIS (Culhane et al. 2007) which covers the XUV range 170-290 $\AA$ (in two bands). This latter range conveniently overlaps the standard spectral selections for normal-incidence imagers such as those on SOHO and TRACE (see above).

The Solar Dynamics Observatory now also provides EUV spectral irradiance measurements for the Sun as a star via its EVE 8 instrument (Woods et al. 2010).

Hard X-rays and $\gamma$-rays: At energies above a few $\mathrm{keV}$, focusing optics have until recently been prohibitively difficult. Accordingly simple counter spectrometers provided the main source of information. Such HXR time series provide important information about the flare impulsive phase (and other epochs of particle acceleration) even without imaging, and many instruments have contributed to this. At present RHESSI and Fermi, through its GBM? instrument (Schwartz et al. 2010) provide this information routinely. New spectroscopic data extending into the $\gamma$-ray range are available from other instruments, including INTEGRAL (e.g., Gros et al. 2004).

Over the years 1991-2001 Yohkoh provided systematic HXR imaging for the first time, using non-focusing optics. The HXT instrument (Kosugi et al. 1991) thus anticipated some of RHESSI's results in HXRs, providing four energy channels over $\sim 15-92 \mathrm{keV}$. True imaging spectroscopy for hard X-rays and $\gamma$-rays began in 2002 with RHESSI (Lin et al. 2002). The single RHESSI instrument consists of nine high-purity germanium detectors, segmented into front and rear segments for sensitive and simultaneous hard X-ray and $\gamma$-ray measurements. These detectors have resolutions as good as about $1 \mathrm{keV}$ (FWHM) over the range $3 \mathrm{keV}$ to $17 \mathrm{MeV}$ (Smith et al. 2002). Each of the detectors has a bigrid modulation collimator with different parameters (thickness and angular resolution) (Hurford et al. 2002), giving imaging (at a basic 4-s time resolution governed by spacecraft rotation) down to the arcsec range.

2.5 Particles and fields

Solar energetic particles: The Sun emits charged particles, neutrons, and energetic neutral atoms as a part of flare/CME physical processes, and these (as well as the solar wind) provide independent samples of material or accelerated particles with which to compare the remotesensing observations. Ideally one would be able to compare the separate populations of energetic electrons and ions detected in situ with those inferred from the RHESSI HXRs and $\gamma$-ray observations. The "multiwavelength" flare observations in a sense include the direct detection of high-energy particles emitted both promptly and with delays by solar flares

\footnotetext{
7 REntgenovsky Spektrometr s Izognutymi Kristalami.

8 EUV Variability Experiment.

9 Gamma-ray Burst Monitor.
} 
and CMEs. These SEPs (Solar Energetic Particles) have a rich history of observation from space, and have also been termed "solar cosmic rays." Many spacecraft have observed SEPs in the past, and the current flotilla includes ACE (Advanced Composition Explorer), SOHO, the two STEREO spacecraft, Ulysses, WIND, and other missions both in deep space and in near-Earth space. The relativistic "ground level event" solar particles can also be detected by neutron monitors at the Earth (e.g., Cliver 2006), either as neutron secondaries or directly in a few cases.

Neutrons and ENAs: Solar neutrons, produced mainly in (p, p), (p, $\alpha)$, and ( $\alpha, p)$ reactions (Hua \& Lingenfelter 1987), can propagate into interplanetary space and even arrive at the Earth's surface if at high enough energies. The natural decay time of a free neutron is about $866 \mathrm{~s} 10$ An array of neutron monitors and neutron telescopes (e.g., Muraki et al. 2007) can detect both direct neutrons and secondary neutrons induced by charged particles creating cascade showers. The largest advances in our understanding of solar neutrons will come from instruments flown within a few tenths of an $\mathrm{AU}$ of the Sun, within the decay-time horizon for lower-energy neutrons that do not survive to one AU.

Neutron-decay products (energetic protons, with a characteristic spectrum) can also be detected in interplanetary space (Evenson et al. 1983, 1990), and in principle fast electrons as well (Dröge et al. 1996). A further channel for flare study has recently surfaced: one event (SOL2006-12-05T10:35, X9) has been found to have emitted detectable levels of energetic neutral hydrogen atoms (Mewaldt et al. 2009) in the 1.8-5 MeV range.

Interplanetary Coronal Mass Ejections: Finally, the low-energy ejecta of an ICME (Interplanetary Coronal Mass Ejection) may contain a large total energy and accelerate particles copiously (see Section 6.2), as well as provide information via their magnetic fields (Burlaga et al. 1982; Bothmer \& Schwenn 1998).

\subsection{Summary}

From the foregoing one can recognize that our observational power has increased considerably during solar cycles 22 and 23, and in fact provides some information in almost all wavelength bands. We list some of the dedicated whole-Sun instruments in Table 2.1 for reference. In spite of all of the data available, it would be quite misleading to imagine that any of the material is definitive, and we return to discuss the missing things in Section 7.2 . The lack of imaging spectroscopy in the optical (other than a few prominent lines) and the $\mathrm{UV}$ is especially painful, and the THz and $\gamma$-ray regimes are very under-exploited. In general one can recognize that non-solar observational facilities (e.g., NASA's Great Observatories such as Chandra) have highly desirable characteristics (resolution, sensitivity, background levels) that are not at present achieved by dedicated solar instruments. In addition, the exciting era of stereoscopic observations has now begun, but with only a limited instrument complement. Clearly stereoscopic observations, including an out-of-the ecliptic capability, would be of great value at all wavelengths; for example, at HXR or radio bands such a capability would make it possible to study the anisotropic radiation patterns of flare emissions, as well as the three-dimensional structures of their sources.

\footnotetext{
10 A free neutron decays, with a half-life of about 15 minutes, into a proton, and electron, and an antineutrino.
} 
Table 2.1 Full-disk solar data sources ${ }^{a}$

\begin{tabular}{|c|c|c|c|}
\hline Observatory & Observation & Dates & URL \\
\hline \multicolumn{4}{|l|}{ I. Radio } \\
\hline Nançay & Decimetric imaging & 1997-present & http://bass2000.bagn.obs-mip.fr/Tarbes/ \\
\hline Nobeyama & $17 \& 35 \mathrm{GHz}$ imaging & 1992-present & http://solar.nro.nao.landac.jp/norh/ \\
\hline SSRT (Irkutsk) & $5.73 \mathrm{GHz}$ imaging & 2000-present & http://ssrt.iszf.irk.ru/ \\
\hline \multicolumn{4}{|l|}{ II. Infrared } \\
\hline Kitt Peak & $8542 \AA, 10830 \AA ̊$ imaging & 2007-present & http://solis.nso.edu/ \\
\hline \multicolumn{4}{|l|}{ III. Optical } \\
\hline GONG & Quasi-continuum images & 1995-present & http://gong.nso.edu/ \\
\hline GONG & LOS magnetic field & 1995-present & http://gong.nso.edu/ \\
\hline Global $\mathrm{H} \alpha$ & $\mathrm{H} \alpha$ Imaging & 2000-present & http://swrl.njit.edu/ghn_web/ \\
\hline$S D O$ & HMI & 2010-present & http://jsoc.stanford.edu/ \\
\hline $\mathrm{SOHO/MDI}$ & Quasi-continuum images & 1995-2011 & http://soi.stanford.edu/ \\
\hline SOHO/MDI & LOS magnetic field & 1995-2011 & http://soi.stanford.edu/ \\
\hline SOLIS & Vector magnetic field & 2008-present & http://solis.nso.edu/ \\
\hline \multicolumn{4}{|l|}{ IV. UV/EUV } \\
\hline SOHO/EIT & $171,195,284,304 \AA$ imaging & $1995-2011$ & http://umbra.nascom.nasa.gov/eit/ \\
\hline STEREO/EUVI & $171,195,284,304 \AA$ imaging & 2006-present & http://secchi.lmsal.com/EUVI/ \\
\hline$S D O$ & AIA & 2010-present & http://jsoc.stanford.edu/ \\
\hline \multicolumn{4}{|l|}{ V. Soft X-ray } \\
\hline Yohkoh/SXT & $\sim 1 \mathrm{keV}$ imaging & $1991-2001$ & http://solar.physics.montana.edu/ylegacy/ \\
\hline GOES-12/SXI & $\sim 1 \mathrm{keV}$ imaging & 2001-2007 & http://www.swpc.noaa.gov/sxi/index.html \\
\hline GOES-13/SXI & $\sim 1 \mathrm{keV}$ imaging & 2006 & http://www.swpc.noaa.gov/sxi/index.html \\
\hline Hinode/XRT & $\sim 1 \mathrm{keV}$ imaging & 2006-present & http://sdc.uio.no/sdc/welcome \\
\hline GOES-14/SXI & $\sim 1 \mathrm{keV}$ imaging & 2009-present & http://www.swpc.noaa.gov/sxi/index.html \\
\hline \multicolumn{4}{|c|}{ VI. Hard X-ray $/ \gamma$-ray } \\
\hline Yohkoh/HXT & $\sim 13-91 \mathrm{keV}$ maging & $1991-2001$ & http://solar.physics.montana.edu/ylegacy/ \\
\hline RHESSI & $3 \mathrm{keV}-17 \mathrm{MeV}$ imaging spectr & 2002-present & http://hesperia.gsfc.nasa.gov/hessi/ \\
\hline
\end{tabular}




\section{Footpoints and ribbons}

\subsection{Overview}

Traditionally we call the HXR brightenings, often observed as paired compact sources, footpoints, and the elongated structures originally found in $\mathrm{H} \alpha$ images ribbons. In fact both features can be observed at many wavelengths; the ribbons are prominent in the UV and EUV (observed, for example, by TRACE (Warren \& Warshall 2001; Saba et al. 2006)). The footpoint sources tend to be distinguished by their strong HXR and/or white light emission. Either way, these features occur pairwise separated by a magnetic polarity inversion line in the photosphere, which separates the two polarities of the vertical magnetic field. Regions connected by coronal magnetic fields are called conjugate points. Figures 1.1, 1.5, and 3.1 show the context.

The principal physical distinction between the footpoint and ribbon morphologies may reflect the idea that the footpoints are the direct result of non-thermal processes (traditionally interpreted in terms of the thick-target model, in which electrons lose their energy collisionally in the chromosphere), whereas the ribbons also show excitation which could be due to thermal conduction from the overlying coronal arcade, or from weak particle precipitation. Careful observation and modeling of individual flare events demonstrates that the location and evolution of the ribbons is clearly related to the magnetic topology in which the event occurs. In particular, flare ribbons reflect the projection of the separatrix or quasi-separatrix structure where flux transfer between magnetic domains is occurring (e.g., Mandrini et al. 1991; Demoulin et al. 1997) (see Section 3.5 for illustrations and further discussion). In $\mathrm{H} \alpha$ and the UV/EUV, the flare ribbons have a tendency to spread systematically outwards from the magnetic polarity inversion line, with their appearance becoming more ordered as the flare progresses.

On the other hand, the HXR footpoint sources frequently move along the $\mathrm{H} \alpha$ or UV/EUV flare ribbons, rather than away from the polarity inversion line, and are found at locations distinguished by their high magnetic field strengths (Temmer et al. 2007) or their high magnetic flux transfer rates (Liu et al. 2008a; Fletcher 2009). HXR footpoints, generated by electron-ion bremsstrahlung of non-thermal electrons in the chromospheric plasma, are well-correlated in space and time with white light sources, confirming them as locations of intense energy deposition (e.g., Hudson et al.2006). Though it was for a long time believed that white-light emission is a "big flare" phenomenon, targeted observations at high cadence and high spectral resolution seem to suggest that even the smallest flares have a white-light counterpart. The generation mechanism for the white-light emission is not known, one issue being that if the white-light emission is a photospheric or lower chromospheric phenomenon this places perhaps unreasonably strong demands on beam excitation models. Observations in the infrared at $1.56 \mu \mathrm{m}$, corresponding to the wavelength at which the solar opacity is a minimum, also suggest strongly that the deep atmosphere is involved (Xu et al. 2004). In EUV, the counterparts to the HXR footpoints are often observed as particularly intense brightenings, and in TRACE observations this leads to characteristic diffraction patterns caused by the metal grids supporting the EUV filters, such that even if the CCD is saturated at the footpoint pixel some limits to its intensity can be calculated (Lin et al. 2001; Gburek et al. 2006; Mrozek et al. 2007).

One of the significant observational discoveries by RHESSI has been $\gamma$-ray footpoints in a small number of solar flares, imaged in the $2.223 \mathrm{MeV}$ neutron capture line (Hurford et al. 2003, 2006). The $2.223 \mathrm{MeV}$ line is produced by the radiative capture on hydrogen of a thermalized neutron. The neutron itself results from an earlier interaction by a primary energetic 


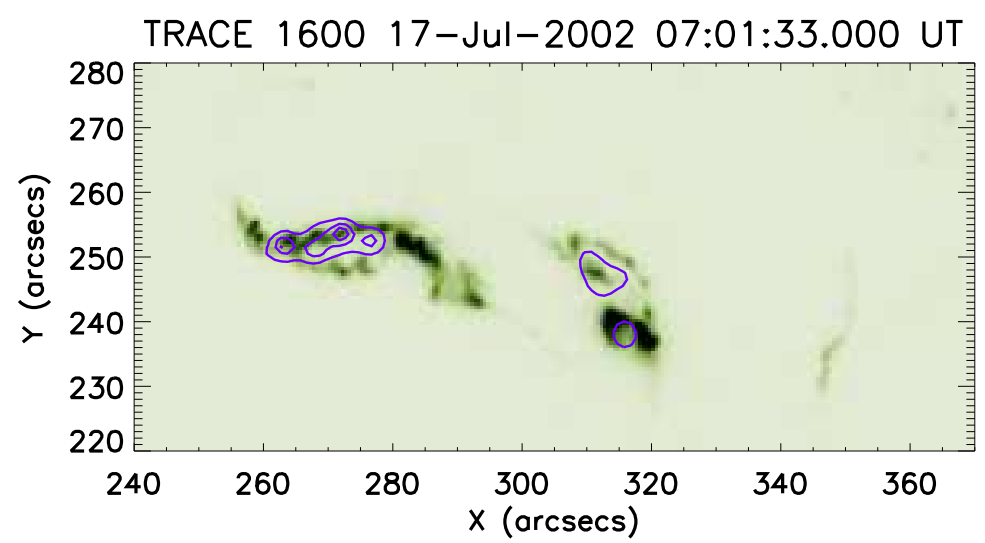

Fig. 3.1 RHESSI and TRACE flare observation of SOL2002-07-17T07:13 (M8.5), showing UV ribbons made up of multiple small sources, and simultaneous RHESSI $25-50 \mathrm{keV}$ footpoints superposed (blue contours, at $25 \%, 50 \%$ and $75 \%$ of maximum). The RHESSI contours were made using the Pixon algorithm (Metcalf et al. 1996), including Grid 1 which is capable of showing spatial detail at $2.3^{\prime \prime}$ scales. The RHESSI sources correspond well to the ribbons overall, but the ribbons are significantly more extended.

ion undergoing inelastic scattering resulting in a neutron-emitting isotope (Hua \& Lingenfelter 1987). It takes a substantial column depth to slow the initially fast neutrons, so the $2.223 \mathrm{MeV}$ radiation presumably forms in the dense lower atmosphere.

The RHESSI 2.223 MeV $\gamma$-ray observations should determine the centroid location of the final neutron captures to within about an arcsecond (Hurford et al. 2003). These observations are difficult to make, involving long integrations because of the low intensity of the radiation, and the high energy photons are modulated only by the thickest RHESSI grids, providing limited spatial information. The four flares that have been imaged in this way so far show single or double (in one case) $\gamma$-ray image components at roughly, but not exactly, the same location as the HXR footpoints. In three out of the four events, there is a statistically significant displacement between the $2.223 \mathrm{MeV}$ sources and the $200-300 \mathrm{keV}$ sources. This is dealt with in Vilmer et al. (2011).

Strong evidence for the impact of solar flares on the deep atmosphere - the lower chromosphere or photosphere - is also present in two other signatures. Flare-induced seismic waves ripple out across the photosphere as detected by helioseismic techniques (Kosovichev \& Zharkova 1998), and strong changes occur in the photospheric magnetic field, most readily visible in the line-of-sight component. The changes are essentially simultaneous with the flare (e.g., Sudol \& Harvey 2005) and with emission at the $1.56 \mu \mathrm{m}$ "opacity minimum" height (Xu et al. 2004). There is no question that the effects of the flare reach deep into the solar atmosphere.

The behavior of the chromospheric footpoint plasma during the impulsive phase remains ill-understood theoretically. At the same time it is in one sense the most important flare problem, since as we will describe in Section 6.2 the white-light and UV continuum in this phase 
may dominate the radiated energy of a flare. The problem has been repeatedly treated in the 1-D "radiation hydrodynamics" approach (Kostiuk \& Pikelner 1975) in which gas dynamics is coupled with some treatment of radiative transfer. Recent developments in this area (Allred et al. 2005) include an elementary formulation of beam heating, along with ionization calculations, line and continuum radiative transfer, and 1-D hydrodynamics. However the limitations of even this advanced treatment (one dimension; no self-consistent treatment of particle or wave energy transport) strongly suggest a need for additional development. The coupling of radiation and matter is decisive for the radiation signatures, of course, and also is necessary to understand the upwards mass flow and the seismic signatures. Selfconsistent modeling should also include wave energy transport (Emslie \& Sturrock 1982; Fletcher \& Hudson 2008).

\subsection{Timing}

The HXR sources can fluctuate rapidly, on time scales below 1 s (e.g., Kiplinger 1995). Such short timescale variations could reflect transport or acceleration processes. For reference, a $30 \mathrm{keV}$ electron at $v=0.3 c$ would move $\sim 10^{4} \mathrm{~km}$ in of order $0.1 \mathrm{sec}$. The timing measurements are especially interesting in comparison with radio measurements, which show myriad fine structures in their spectrograms. Figure 3.2 shows a representative example of a decimeter-wavelength dynamic spectrum (unflitered and frequency-filtered) compared with HXRs (Aschwanden et al. 1995a), in which detailed correlation between spikes is seen. It is more difficult to interpret the decimeter bursts because of their non-linear generation mechanisms; the timescale for the development of the kinetic instability related to the beam transport time (the "bump-on-tail" instability) is important. In some flares the decimeter wavelengths and HXR correlations are very good, but not in all - possibly due to the complexities of the plasma radiation generation and absorption/Razin suppression (Bastian et al. 1998). At centimeter wavelengths there can be a much more precise relationship.

Sakao et al. (1996) have used HXR timing measurements to establish conjugacy (see Section 3.1) statistically, drawing the inference that a coronal particle acceleration region sends bremsstrahlung-producing fast electrons simultaneously (to a few tenths of a second) into the two footpoint regions. At present there is no analysis of RHESSI data along these lines. Aschwanden et al. (1995b) have extended HXR timing analysis in an effort to establish time-of-flight delays between HXRs at different energies. Their decomposition of the time series of the Masuda flare, to name only one example, is consistent with signatures of looptop injection superposed on a slowly varying envelope; note though that the interpretation of such a decomposition is not unique (Brown et al. 1998).

\subsection{Morphology of flare footpoints and ribbons}

There are distinctions between the impulsive (early) and gradual (late) phase behavior of flare ribbons and footpoints. In the gradual phase of a flare, the emission tends to be in two roughly parallel ribbons, visible particularly well in $\mathrm{H} \alpha$ and UV (see, for example, Figure 3.3. These tend to separate slowly from one another and from the magnetic polarity inversion line as the flare proceeds, although the HXR footpoints can have more complicated apparent motions as discussed below. The roughly parallel expansion of the ribbons led to the standard magnetic reconnection model. In the gradual phase of the flare, footpoint emission is thought to be powered primarily by electron beams, but may instead result 

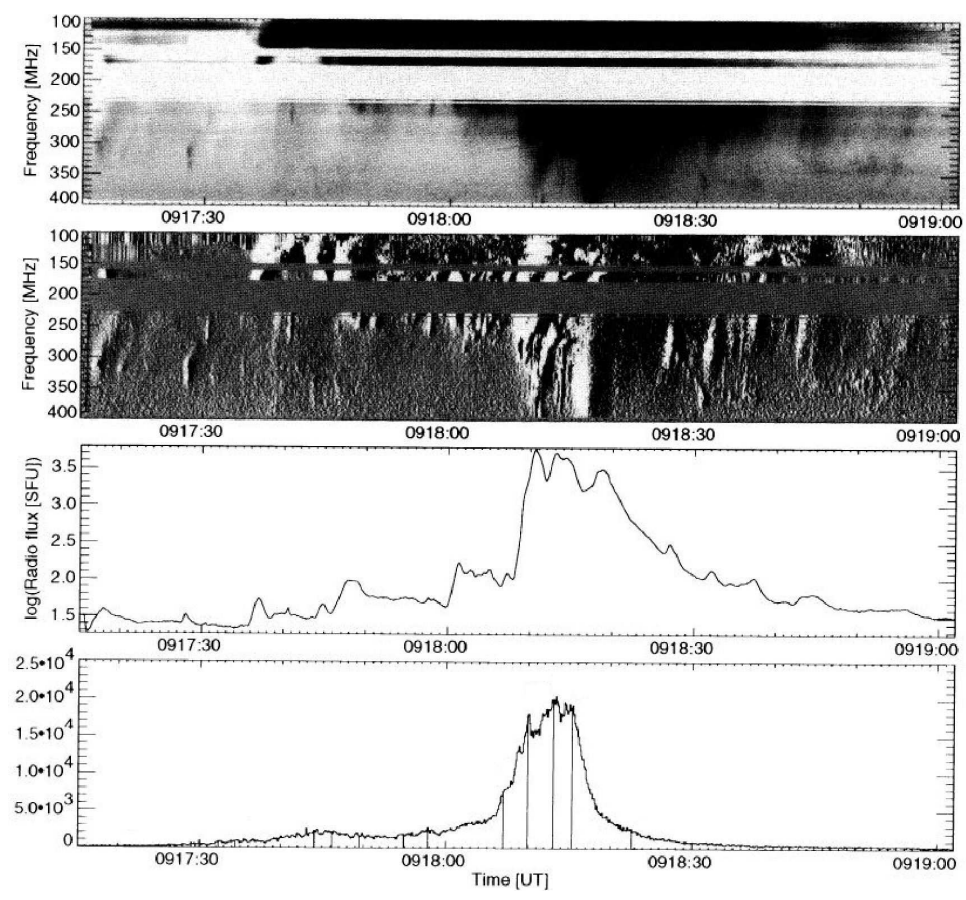

Fig. 3.2 Comparison of decimeter-wave and HXR time variations (Aschwanden et al. 1995a); data from the Ikarus spectrograph (Perrenoud 1982) and the SMM/HXRBS instrument (Orwig et al. (1980), respectively. The upper two panels show the radio spectrogram for SOL1980-03-29T09:18, with the lower one filtered to show temporal gradients; the bottom two panels show the radio flux integrated over $100-400 \mathrm{MHz}$ and the HXR flux.

from thermal conduction from the overlying hot loops (see the analysis of Czaykowska et al. 2001). The flare ribbons in $\mathrm{H} \alpha$ can be some tens of arcseconds wide, particularly in the gradual phase, and show internal structure (also visible in some cases in UV). The gradual phase ribbon morphology in EUV appears similar to a patterning associated with hot, high pressure coronal loops (in this case flare loops). In such structures conduction from the loops causes plasmas at transition-region or coronal temperature to appear at chromospheric heights. The resulting pattern of spatially-intermittent and dynamic hot plasma and spicules is known as "moss" (Berger et al. 1999). The leading edges of the ribbons, illuminated in EUV, are considerably narrower (e.g., Asai et al. 2003), and are also where the brightest $\mathrm{H} \alpha$ emission (Švestka et al. 1982) and the HXR footpoint sources are found in the impulsive phase. Hard X-ray bremsstrahlung is not normally a feature of the ribbon-associated gradual phase, athough late-phase non-thermal emission with different morphologies is now known to be common (Krucker et al. 2008a).

In the flare impulsive phase the picture is more complicated. For example, more than two UV or $\mathrm{H} \alpha$ ribbons may be seen in magnetically quadrupolar flares, (e.g., Tang 1985; Su et al. 2007), as well as a small number of bright footpoints visible in white-light, UV, $\mathrm{HXR}$ or $\mathrm{H} \alpha$ kernels. In general the HXR sources are confined to localized areas situated on the outer edges of the elongated flare ribbons observed in UV and $\mathrm{H} \alpha$ (Figure 1.5) and are predominantly associated with bright H $\alpha / \mathrm{UV}$ kernels Asai et al. 2002, 2004a; 


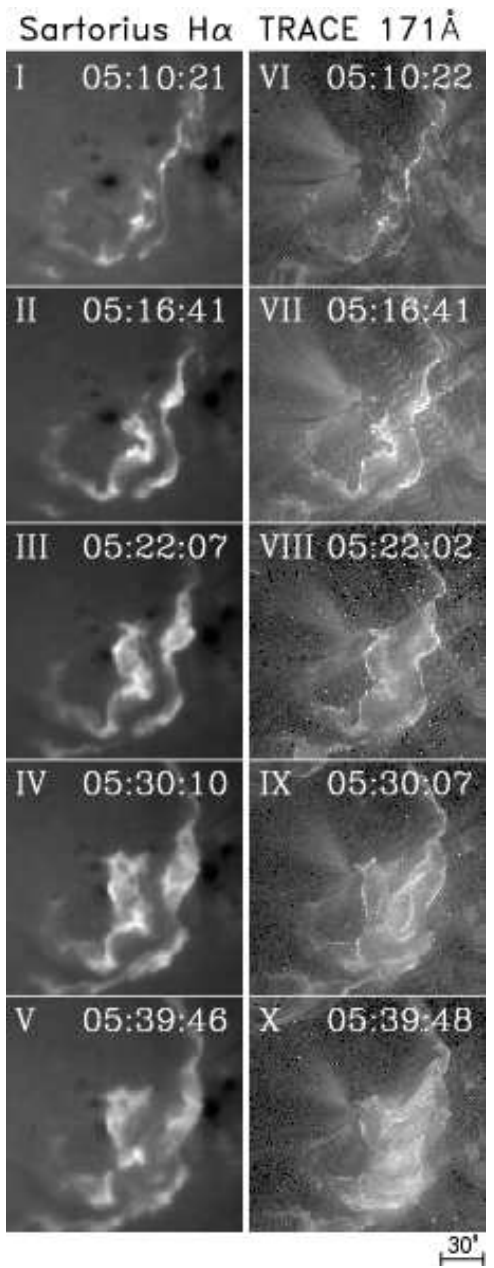

Fig. 3.3 The flare SOL2001-04-10T05:26 (X2.3) (Asai et al. 2003) in $\mathrm{H} \alpha$, showing chromospheric emission, and TRACE $171 \AA$ showing a mixture of transition-region ribbons and loops. This sequence shows clearly the spreading of the flare ribbons as the flare progresses. This event had an extended impulsive phase consisting of several HXR spikes; the cross diffraction patterns at the footpoints are characteristic of TRACE impulsive phase EUV observations. The thickening of the $\mathrm{H} \alpha$ ribbons is clear, and where these thickened inner portions map to the TRACE emission in panel VII, there is a "moss"-like appearance.

Krucker et al. 2005). Often there are two main HXR footpoints, but sometimes there are more (e.g., Fletcher \& Hudson 2002; Lin et al. 2003; Temmer et al. 2007). The HXR emission is direct confirmation of the presence of non-thermal electrons in the lower chromosphere, which also heat and (further) ionize the chromospheric plasma as they stop collisionally.

A basic property of flare footpoints is that they are compact. In wavelengths from the infrared through the extreme ultraviolet, the dimension of the brightest flare footpoints matches the miniumum scale resolvable by the instrumentation used. Only with the launch of Hinode do we have hints that we are approaching the basic scale for the optical flare 
kernels. Sub-arcsecond structure has been detected in optical flare sources which are seen to consist of a bright emission core with a FWHM of around $500 \mathrm{~km}$ (corresponding to an area of around $10^{16} \mathrm{~cm}^{2}$ ), surrounded by a diffuse halo of emission having greater extent (Isobe et al. 2007). This diffuse halo is interpreted as radiation from the core backscattered by deeper atmospheric layers. Arcsecond-scale widths (diffraction limited) for flare ribbons were also observed in the infrared at $1.56 \mu \mathrm{m}$ (Xu et al. 2004). Spatial resolution at UV/EUV wavelengths is not so high, but TRACE UV observations of flare kernels in the $1600 \AA$ band, where not saturated, are consistent with them being on the scale of the telescope point spread function (see images in e.g., Warren \& Warshall 2001; Alexander \& Coyner 2006). The size scale of HXR images is harder to quantify, but in some flares RHESSI imaging reveals HXR footpoint sizes comparable with the resolution capability of the finest grids (2.3 arcseconds FWHM) (e.g., Fletcher et al. 2007; Schmahl et al. 2007; Kontar et al. 2008; Dennis \& Pernak 2009), corresponding to an area on the order of $10^{17} \mathrm{~cm}^{2}$. The smaller optical sizes may imply that the optical excitation is taking place deeper down, in a converging magnetic field, or it may mean that the HXR footpoints simply have not been resolved yet at the best RHESSI resolution.

During the Yohkoh era, the Yohkoh/SXT observed impulsive SXR footpoint sources indicating heating of upper chromosphere or transition region plasmas to around $10 \mathrm{MK}$ (McTiernan et al. 1993; Hudson et al. 1994; Mrozek \& Tomczak 2004). The SXR emission is not consistent with an extrapolation of the bremsstrahlung power law spectrum to low energies, and originates in the chromosphere. Thus it appears to correspond to strong heating of the chromosphere (Hudson et al. 1994). In beam-driven radiative hydrodynamic simulations, to achieve such temperatures at the appropriate heights in the atmosphere requires beam energy fluxes on the order of $10^{11} \mathrm{erg} \mathrm{cm}^{-2} \mathrm{~s}^{-1}$, or alternatively a lower electron flux for hundreds of seconds (Allred et al. 2005).

Although the impulsive-phase HXRs and optical emissions are well-correlated in space and in time, the relationship between HXR and UV/EUV emission is not so clear. There are often pre-flare brightenings in UV which remain bright during the flare, and the HXR footpoints occur only at locations which were not bright in UV before the flare (Warren \& Warshall 2001). There are good temporal UV/HXR correlations during the flare, but as noted the UV ribbons are more extended than the HXR footpoints (Alexander \& Coyner 2006). So there are evidently only a few locations in the flare magnetic field which are involved in the acceleration of a large number of non-thermal particles. Looking next at the UV/EUV and HXR sources which are at the same locations during the flare itself, there is a relatively good correlation between the TRACE $1600 \AA$ channel flux and the Yohkoh/HXT 33-53 keV flux, and a weak anticorrelation between the TRACE UV (1600 $\AA)$ and Yohkoh HXR spectral index (Mrozek et al. 2007). An anticorrelation would be expected due to the plasma heating in the upper chromospheric levels produced by an electron beam in which low energies (with short collisional stopping depths) dominate. The TRACE $1600 \AA$ channel is rather broad in wavelength, so it is not clear which lines or continua may be dominating the UV flux. This may explain in part the relative weakness of this correlation. However, the weak correlation may well indicate the importance of heating mechanisms other than beam heating (e.g., thermal conduction) in producing EUV footpoints. The study has not yet been repeated using the better spectroscopic capabilities offered by RHESSI.

A small number of isolated footpoints is the dominant impulsive-phase HXR morphology, with elongated HXR flare ribbons rarely observed. The first report of HXR ribbons was in Yohkoh HXT observations of SOL2000-07-14T10:24 (X5.7) (Masuda et al. 2001). In the well-observed SOL2005-05-13T16:57 (M8.0) flare, the RHESSI HXR sources evolve from footpoints concentrated in strong magnetic field areas along the TRACE UV ribbons in 

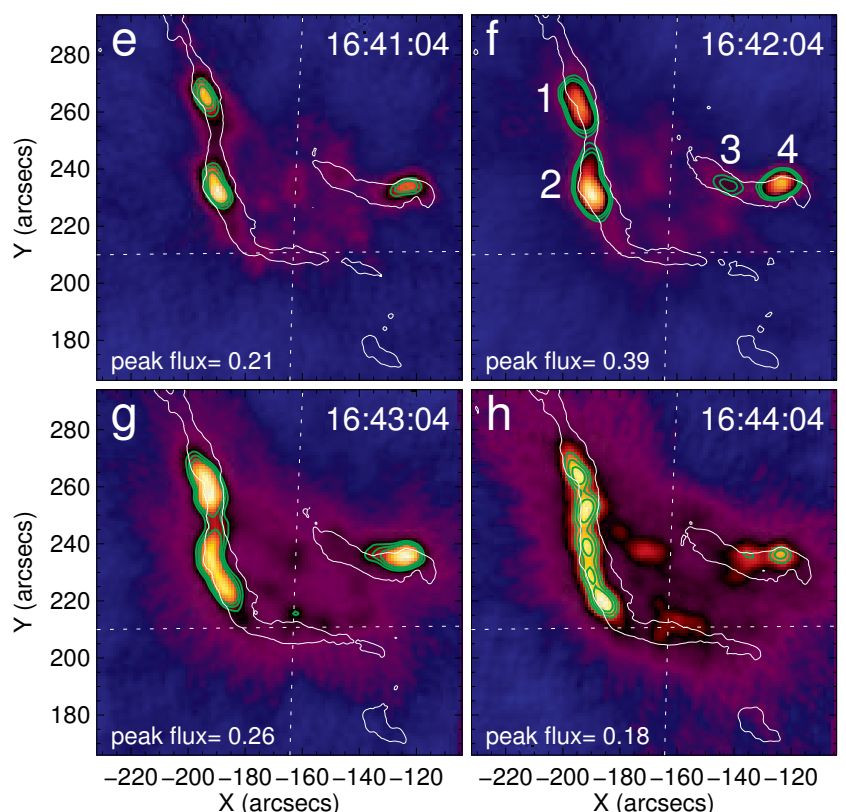

Fig. 3.4 Time sequence of RHESSI 25-50 keV HXR images together with TRACE $1600 \AA$ image contours (white lines) showing the evolution of the HXR source emission from localized footpoints to ribbon-like emission. Flare SOL2005-05-13T16:57 (M8.0), adapted from Liu et al. (2007).

the HXR rising phase to ribbon-like HXR source structures closely matching the UV flare ribbon morphology after the HXR peak (see Figure 3.4). The simpler structure of the HXR ribbons presumably reflects more closely the pattern of electron acceleration along the flare loop arcade during its formation (Liu et al. 2007, 2008a). Figure 3.4 suggests that RHESSI has sufficient resolution to resolve discrete footpoint features that would be interpreted as multiple simultaneous footpoint brightenings. We should note that the appearance of elongated HXR ribbon-like features might also result from source motions during the relatively long HXR integration times ( $>4 \mathrm{~s}$ for RHESSI imaging).

\subsection{Motions of ribbons and footpoints}

As flare reconnection proceeds in the standard model, different elements of the magnetic field move into the reconnection region. This leads to the expectation that the H $\alpha / \mathrm{UV}$ flare ribbons or footpoint sources move. In many flares the footpoints appear to move away from one another and from the magnetic polarity inversion line as the flare loop system grows (for recent studies see, e.g., Fletcher \& Hudson 2002; Oiu et al.|2002; Krucker et al. 2003; Asai et al. 2004a; Veronig et al. 2006; Miklenic et al. 2007; Temmer et al. 2007). The flare ribbons are understood to somehow map to the energy release site in solar flares, and the movement of the ribbons and kernels across the photosphere, and their relationship to magnetic fields, is an important means by which the magnetic reconnection process can be explored. For example, under the assumption of magnetic flux conservation the progress of 
the flare sources across the magnetic flux of the photosphere can be used to measure the magnetic flux transfer rate.

However, much more complex HXR footpoint motions are observed than the straightforward separating ribbon motions mentioned above. In Yohkoh/HXT data only about 13\% of HXR flares exhibit HXR footpoint motions corresponding strictly to separation with respect to the polarity inversion line (Bogachev et al. 2005), and are more likely to have a component of motion along the ribbon direction. They can also approach one another. Both converging and separating footpoints travel at tens of kilometers per second. A recent statistical study of footpoint motions in 27 RHESSI X- and M-class flares by Yang et al. (2009) has found that parallel/antiparallel motions are more likely during the SXR rise phase than during the flare peak, where separating motions become more prominent. Many individual examples confirm that the HXR footpoint motions do not always agree with the standardmodel predictions of separating footpoints (e.g., Fletcher \& Hudson 2002; Krucker et al. 2003; Grigis \& Benz 2005; Ji et al. 2006). As in the 2-D flare model, the interpretation of the footpoint motion is still in terms of magnetic reconnection, but in a complex magnetic field. The complicated footpoint motions are likely to be linked to the projection(s) of the locus of reconnection. Particular examples of this can be found in sheared arcade models (Somov et al. 2002), and the "slip-running" reconnection model (Aulanier et al. 2006).

Footpoint motion parallel to the magnetic polarity inversion line: An example of footpoint motion parallel to the magnetic inversion line is shown in Figure 3.5. The SOHO/EIT image of SOL2002-11-09T13:23 (M4.9) shows a postflare arcade, with the centroid position of the RHESSI HXR footpoints superimposed. As the event progresses, in several emission spikes, the HXR footpoint pairs move along the arcade. Source motion specific to the emission spikes shows up as deviations from the overall trend of the footpoint motions, and are decomposed into parallel and perpendicular components relative to this trend. Footpoint motion is directed parallel to the ribbons and is smooth at these scales, in contrast with the bursty evolution of the HXR flux. There is no evidence for a systematic trend in outward perpendicular displacement, or of discontinuities during the transitions from one spike to the next. The emission spikes originate at different sources along the arcade. The overall picture is that the HXR footpoint motion may be a consequence of a moving trigger, possibly caused by an asymmetric eruption of a filament (Tripathi et al. 2006), or a "domino effect" where energy release in part of the field triggers activity in its neighbors.

Converging Footpoints: In its first couple of minutes, SOL2002-11-09T13:23 (M4.9) also exhibits a convergence of the flare footpoints, explained in terms of the sequential activation of a flare arcade which varies in width along its length (Grigis \& Benz 2005). A different kind of converging footpoint motion, corresponding to footpoints travelling anti-parallel to one another and along the ribbons, was seen earlier with Yohkoh/HXT (Bogachev et al. 2005), and now frequently in RHESSI (e.g., Fletcher \& Hudson 2002). However, a new feature of such events became apparent in RHESSI observations showing the accompanying HXR coronal source. A number of events observed in HXRs, $\mathrm{H} \alpha$ and UV/EUV showed approaching footpoints in the early impulsive phase, accompanied by a projected downward motion of the coronal HXR source, and followed by separation of the footpoints and a projected rise in the coronal source (Figure 3.6). These include SOL2002-03-14T01:44 (M5.7), SOL2003-10-29T20:49 (X10.0) (Zhou et al. 2008; Ji et al. 2008; Liu et al. 2009b), SOL2002-09-09T17:52 (M2.1), and SOL2004-11-01T03:22 (M1.1) (Ji et al.|2004, 2006). The initial converging motion has also been noted in UV/EUV ribbons (Zhou et al. 2008). The footpoint convergence phase lasts for a few minutes, and both footpoints and coronal 

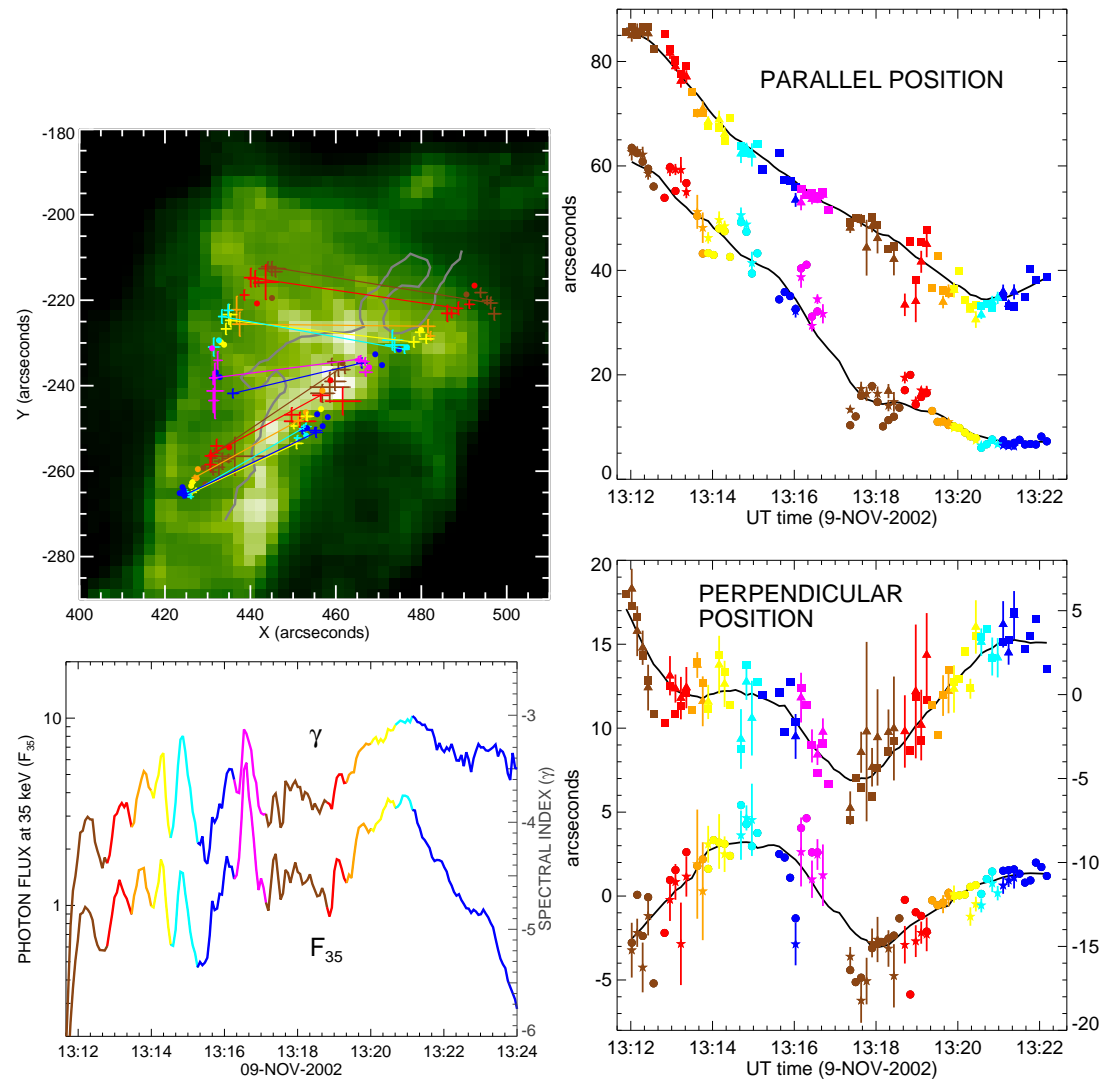

Fig. 3.5 Top left: SOHO/EIT $195 \AA$ image of post-flare loops with the RHESSI HXR source positions superimposed for SOL2002-11-09T13:23 (M4.9). The positions of the 20-50 keV sources from the CLEAN images are represented by crosses with arm lengths equal to the errors, positions from the PIXON images are given by circles. Simultaneous footpoints are connected and color coded according to the time intervals defined in the bottom part. The neutral line is shown in gray. Bottom left: Time evolution of the flux and spectral index. Right: Time evolution of the source positions relative to the trend lines. Triangles and stars with error bars refer to values derived using CLEAN, squares and circles using PIXON, for the western and eastern footpoints, respectively.

sources move with a projected speed of some tens of $\mathrm{km} \mathrm{s}^{-1}$. Liu et al. (2009b) found an example of simultaneous height decrease and footpoint convergence. In the cases of antiparallel footpoint convergence, the empirical shear (determined from the angle relative to the neutral line made by the line joining footpoints) may decrease no matter whether the footpoints move inward or outward with respect to the polarity inversion line.

The downward motion of the coronal sources is consistent with the extraction of magnetic energy from the field (Hudson 2000). It has been interpreted in two related ways as an initial shrinkage of the field in a 2-D "collapsing trap" immediately following reconnection (e.g., Karlický \& Kosugi 2004; Veronig et al. 2006) and as the consequence of the relaxation of shear in a 3-D arcade model (see also Ji et al. 2007; Somov et al. 2002). In the latter model, the less-sheared field reaches a lower altitude in the corona than more-sheared field, and its relaxation can in principle explain both the decrease in coronal source altitude 


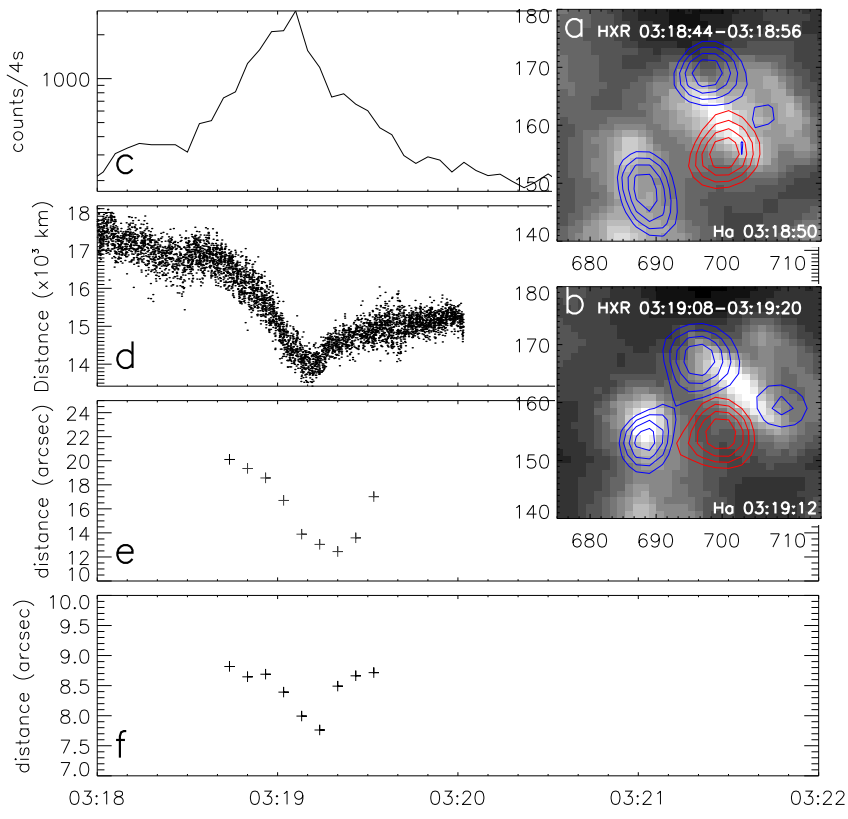

Fig. 3.6 Converging footpoints in SOL2004-11-01T03:22 (M1.1): (a)-(b) RHESSI HXR contours overlaid on $\mathrm{H}_{\alpha}-0.5 \AA$ filtergrams taken at PMO, with the blue contours at $25-60 \mathrm{keV}$ and the red ones at 3-6 keV, (c) RHESSI 50-100 keV time profiles, (d) distance between the two conjugate $\mathrm{H} \alpha$ kernels or HXR conjugate footpoint sources, and e) projected height of the RHESSI loop-top source. From Ji et al. (2006).

and the converging footpoint motion. We discuss the relationship between ribbon motions and coronal dynamics extensively in Section 4.6 below.

\subsection{Flare footpoints and the magnetic environment}

It has long been known that the magnetic structure of the solar corona is reflected in the distribution and evolution of flare footpoint sources. The simplest example of this is the straightforward mapping between the pre- and post-reconnected field in the 2-D standard model, and the spreading $\mathrm{H} \alpha$ ribbons. Even in more magnetically complex configurations, in principle each X-ray footpoint, or white-light/UV kernel, maps via the coronal field to a conjugate counterpart. In practice this has been difficult to demonstrate quantitatively. The flare impulsive phase is characterized by complex magnetic geometries, and recent years have also seen great advances in breaking down the active region coronal field into its topological elements - separatrix and quasi-separatrix layers, separator field lines and null-points. This remains an area of intense theoretical activity, as part of an overall effort to understand how magnetic reconnection takes place in three dimensions. The hope is that the observed evolution of footpoints can aid in this overall goal. During the flare impulsive phase, where the HXR and WL footpoints are typically observed, the magnetic geometry is not readily interpreted from EUV loop observations (as is the case in the gradual phase). The magnetic field is presumably stressed, and therefore the relatively straightforward potential field extrapolations may provide a misleading picture of the the overall coronal structure 


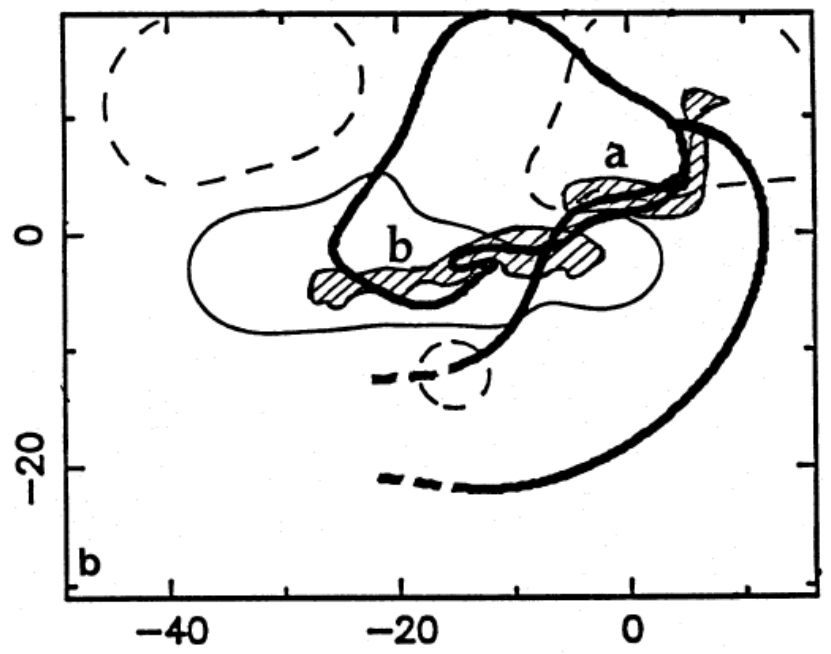

Fig. 3.7 Portions of the photospheric projection of separatrix structures from a linear force-free magnetic extrapolation are shown by Mandrini et al. (1995) to correspond to the locations of flare $\mathrm{H} \alpha$ ribbons. Contours show $\pm 400 \mathrm{G}$ levels of magnetic field strength. Note, the correspondence between separatrix and ribbon positions is not found for a potential model. The $\mathrm{H} \alpha$ ribbons are found by Mandrini et al. (1995) to correspond to the regions of highest current density in their model.

(though as we see below they have been used to explore certain aspects of the flare geometry). Since the Skylab era (Zirin \& Tanaka 1973; Pallavicini et al. 1975) it has been clear that later loops in the gradual phase of a flare look more potential-like, and make a larger angle to the neutral line, and this is often glibly taken as evidence for the reduction of shear expected to reduce the stored magnetic energy. Clearly it is not that simple; the observed changing pattern of shear is determined by the amount of shear in the pre-flare field as a function of distance from the neutral line before the flare, as well as its reduction as a function of time during the flare. The distribution of magnetic shear within the flaring volume reflects the paths taken by coronal current systems, about which we have little knowledge.

Footpoints and magnetic topology: The earliest observational studies demonstrated that the locations of $\mathrm{H} \alpha$ ribbons could be explained (with a suitable arrangement of magnetic charges 11 , extrapolated in a potential approximation) as the intersection of coronal separatrix surfaces with the photospheric boundary (Gorbachev \& Somov 1989; Mandrini et al. 1991; Longcope 1996). An example of this is shown in Figure 3.7 (Mandrini et al. 1995). Many flares have been modeled using similar approaches with increasing degrees of complexity - for example, incorporating linear force-free fields (Demoulin et al. 1994) and non-linear force-free fields (Régnier et al. 2002; Régnier \& Canfield 2006; Schrijver et al. 2008), and using increasingly precise representations of the photospheric field (Barnes et al. 2005) and chromospheric magnetic fields (Metcalf et al. 2005) as input. Overall the correlations between the photospheric mappings of separatrices, quasi-separatrices and separators, and the observed location of flare footpoints are convincing, but certain aspects still evade a clear

11 This is "magnetic charge topology," in which an array of fictitious magnetic monopoles is used as a best fit to a photospheric magnetogram, as a basis for 3-D potential-field extrapolations Baum \& Bratenahl 1980; Demoulin et al. 1993; Longcope 1996). 

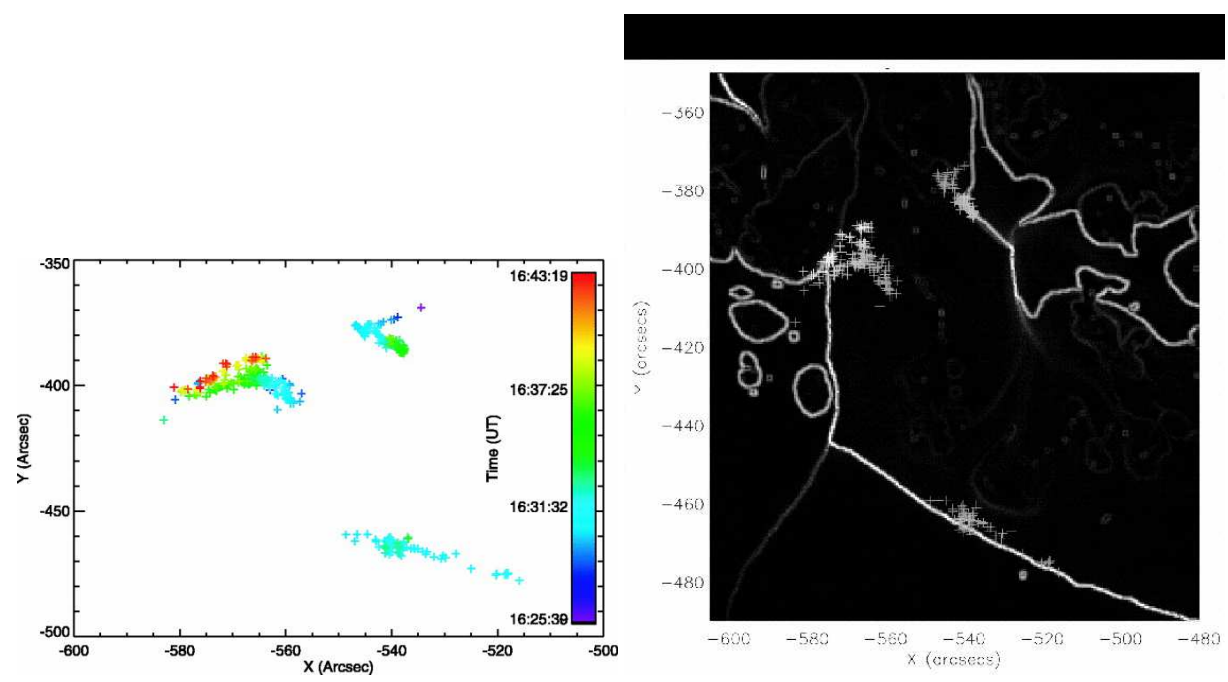

Fig. 3.8 Left: positions of the HXR footpoints in SOL2001-08-25T16:45 (X5.3) (Metcalf et al. 2003), with time color-coded on the right; data from the Yohkoh/HXT instrument (Sakad 1994). Right: locations of the separatrix surfaces projected onto the photospheric plane, with the shading representing the magnitude of the separatrix discontinuity. The upper footpoints illustrate the complex motions along the flare ribbons, while the lower points show rapid motion. The authors showed that this motion coincided with the photospheric intersection of a magnetic separatrix structure.

explanation. These mappings have not yet allowed us to fully understand the reason why the HXR or WL footpoints are few and compact, while the $\mathrm{H} \alpha$ ribbons are extended, nor the properties of the reconnection that determine the motions of the footpoints. But they are providing us with fascinating clues.

Prior to RHESSI, a small number of studies had been carried out in which the relationship between magnetic field and HXR footpoint location was investigated. As well as the investigations into footpoint asymmetry described below, there was some early work on HXR sources in the context of magnetic topology. For example, Figure 3.8 shows the Yohkoh HXR and TRACE WL source motions observed in SOL2001-08-25T16:45 (X5.3), compared to the projections of coronal separatrix structures onto the photosphere (Metcalf et al. 2003 ). This Figure reveals strong resemblances between observed and calculated features; in particular the lower source moves extremely rapidly almost in coincidence with one of the separatrix intersections, in a manner suggesting the "slip-running" reconnection model of Aulanier et al. (2006). Evidence was also found for a coronal null and reconnection of external field through a separatrix "dome" (Fletcher et al. 2001). Yohkoh-era observations also showed that HXR sources tended to avoid sites of high vertical current density, preferentially occurring adjacent to them (Li et al. 1997).

RHESSI data have led to more studies relating HXR footpoint behavior and magnetic fields. For example, in SOL2005-01-17T09:52 (X3.8), which exhibits four $\mathrm{H} \alpha$ ribbons and corresponding HXR footpoint sources, the two strongest and long-lived $\mathrm{H} \alpha$ kernels and HXR footpoints are observed to tend to avoid the strongest fields, and move approximately along iso-Gauss contours, along the border between the sunspot's umbra and penumbra (Temmer et al. 2007). Also in this event, the magnetic reconnection rates derived for flare 

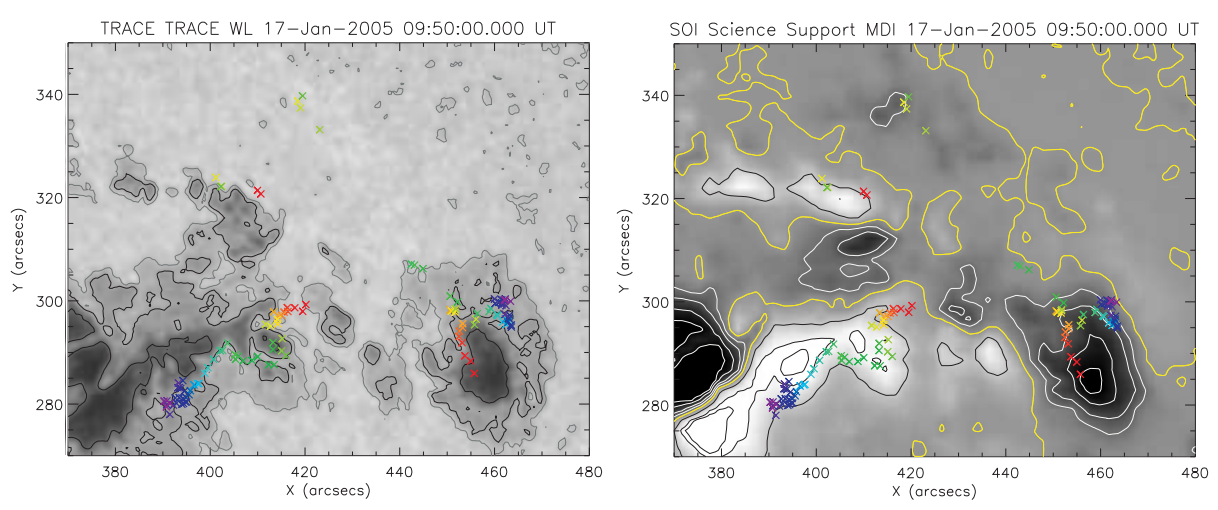

Fig. 3.9 RHESSI hard X-ray source centroids overlaid on a TRACE white-light image (left) and MDI magnetogram (right) for the X4 flare SOL2005-01-17T09:52. The subsequent occurrence of the HXR sources from 09:43:20 to 10:04:10 UT is color-coded from red to blue. Left: TRACE white-light contours roughly outline the umbrae as well as inner and outer penumbrae of the sunspots. Right: Isogauss lines at $-2000,-1600,-1300$, and $-600 \mathrm{G}$ (white contours) and $+600,+1300$, and $+1500 \mathrm{G}$ (black contours). The yellow line marks the magnetic inversion line. Adapted from Temmer et al. (2007).

ribbon locations showing HXR footpoints are higher (by two orders of magnitude) than those in flare ribbon locations not showing HXRs. The strongest HXR sources were preferentially located in those regions of the ribbons with the strongest magnetic field, although this cannot readily be seen from Figure 3.9. Similar results were obtained from Yohkoh/HXT studies (Asai et al. 2002, 2004a).

For three major RHESSI flares, it has been demonstrated (Des Jardins et al. 2009) that the path of HXR footpoints corresponds to a particular type of topological structure, a subset of the photospheric spine lines identified in magnetic charge topology models (see Longcope, 2005, and the discussion in Section 3.5). These are lines that join two magnetic sources of the same sign ("charge") via a magnetic null. The implication is that the footpoint movement reflects the changing length of the separator joining the nulls on the two spine lines, as the coronal reconnection proceeds and the reconnection region moves.

The huge differences derived in the local energy release rates for flare ribbon locations with/without HXR footpoints in combination with the limited dynamic range of present HXR instruments (of order 10:1) can explain the different flare morphologies typically observed in HXRs (compact footpoints) and H $\alpha / \mathrm{UV}$ (extended ribbons). However, it is still implied that a large fraction of the electrons is accelerated into spatially confined subsystems of magnetic loops as outlined by the HXR footpoints, and only a minor fraction goes into the large flare arcade outlined by the H $\alpha / \mathrm{UV}$ ribbons and EUV postflare loops (Temmer et al. 2007).

Although white light is an important indicator of the locations of strongest energy input (e.g., Neidig 1989), and although it is substantially simpler to image than HXRs, we do not have adequate systematic observations. In particular the white-light footpoint motion has only rarely been studied. SOL2001-08-25T16:45 (X5.3) (Metcalf et al.2003) is one such example. The SOL2002-09-30T01:50 (M2.1) white-light flare studied by Chen \& Ding (2006) is another. It showed systematic footpoint motion in the white-light continuum, following roughly that of the corresponding HXR source. Footpoints at both wavelengths zigzag back and forth, primarily parallel to the magnetic neutral line (see Figure 3.10) in a manner which may be explained by the particular magnetic configuration in the flaring region. 


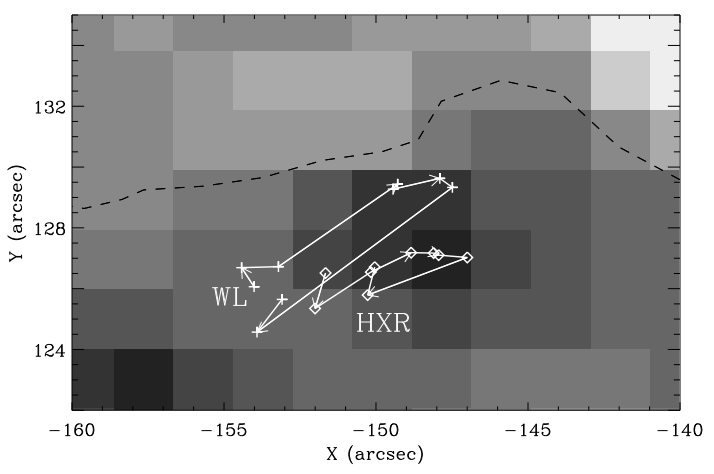

Fig. 3.10 Footpoint motion history in the white-light continuum (pluses) and 12-25 keV HXR emission (diamonds) superposed on the MDI magnetogram in the white-light flare SOL2002-09-30T01:50 (M2.1). The magnetic neutral line is plotted as the dashed line (Chen \& Ding 2006). The points generally describe a clockwise motion, and cover a time interval of about five minutes.

Footpoint Asymmetry: A basic prediction of flare models invoking electron acceleration in the corona and precipitation to the chromosphere is that regions with stronger magnetic field convergence (i.e., a stronger chromospheric or photospheric magnetic field) should be locations of weaker HXR footpoint sources, because the higher mirror ratio leads to a larger fraction of accelerated electrons mirroring before they reach the thick-target footpoints. The ratio of brightness in footpoint pairs should thus be inversely correlated with the ratio of magnetic field strengths at the location of those footpoints. This tendency was demonstrated systematically in early analyses of a small number of double-footpoint flares observed with Yohkoh/HXT, using both line-of-sight (e.g., Sakao et al. 1994) and vector fields (Li et al. 1997), but later work revealed counter-examples. Stronger HXR footpoints were found in stronger magnetic field regions in at least one-third of 32 flares examined (e.g., Goff et al. 2004). A detailed study by Liu et al. (2009b) of RHESSI footpoint pairs in SOL2003-1029T20:49 (X10.0) showed the expected relationship in the first few minutes of the event, but thereafter it disappeared. Furthermore, although the sign of the correlation was as expected early in the event, the magnitude was not consistent with simple predictions of the magnetic mirroring model. They also found that collisional losses due to asymmetric column densities from the looptop (assumed to be the acceleration region) to the footpoints alone cannot explain the totality of the observed HXR fluxes and spectra. This is consistent with the result of a statistical study of RHESSI footpoint asymmetry carried out by Saint-Hilaire et al. (2008), though these authors did not examine the footpoint magnetic fields. As Liu et al. (2009c) suggest, more detailed modeling including mirroring, collisional losses, and other particle transport effects (such as nonuniform target ionization, relativistic beaming, photospheric albedo, and return currents) may provide a resolution to the above discrepancies (see Figure 3.11). An alternative investigation of the footpoint asymmetry intrinsic to the acceleration process has been pursued (McClements \& Alexander 2005) to explain the observations of Alexander \& Metcalf (2002).

Most of the microwave emission in flares is gyrosynchrotron from non-thermal electrons, the intensity of which depends on the electron energy and on the ambient magnetic field. Thus the stronger-field footpoint should correspond to stronger microwave sources (and weaker HXR footpoints), giving a complementary view of footpoint asymmetry. This 


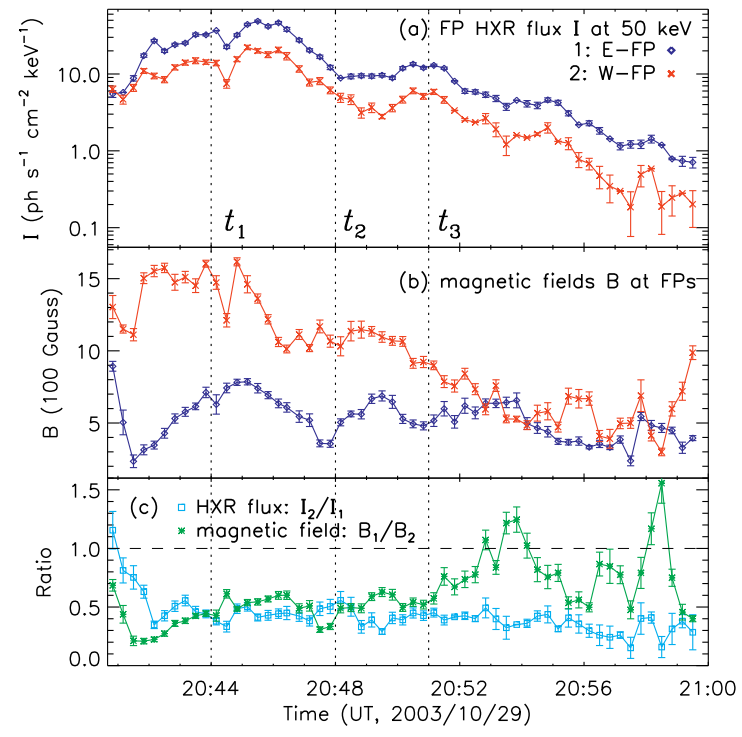

Fig. 3.11 Time profiles of X-ray and magnetic field parameters of the conjugate footpoints in SOL2003-1029T20:49 (X10.0). (a) HXR fluxes at $50 \mathrm{keV}$ of the eastern footpoint (E-FP, blue diamonds) and western footpoint (W-FP, red crosses) obtained from power-law fits in the 50-150 keV range. (b) SOHO/MDI magnetic field strengths registered at the two footpoints. (c) Ratios of the $50 \mathrm{keV}$ fluxes (W-to-E) and magnetic fields (E-to-W) of the two footpoints. The expected correlation between these two ratios only holds for the first half of the flare duration (from Liu et al. 2009b).

relationship has been found to hold in some recent investigations (Kundu et al. 1995; Wang et al. 1995), but the modeling presents the complicated problem of understanding the microwave absorption. Without understanding absorption it is not possible to get at information about the microwave footpoints, and so any conclusions drawn from HXR and microwave comparisons are premature.

Inference of the properties of magnetic reconnection: The product of the footpoint apparent speed and the line-of-sight magnetic field, expressed as a flux transfer rate $\dot{\Phi}$, has been used as a measure of the coronal reconnection rate by various authors (e.g., Oiu et al. 2002; Fletcher et al. 2004; Jing et al. 2005; Temmer et al. 2007). The quantity $\dot{\Phi}$ can be estimated from the observations as (e.g., Forbes \& Lin 2000):

$$
\dot{\Phi}=\frac{\partial}{\partial t} \int B_{\mathrm{ph}} d a
$$

where $d a$ denotes the ribbon area and $B_{\mathrm{ph}}$ the normal component of the photospheric magnetic field.

In the gradual phase of large two-ribbon flares where the magnetic configuration is wellapproximated by a 2- or 2.5-D field (i.e., no significant shear or twist component of the field), the coronal reconnection rate is also equal to an equivalent electric field $E_{c}$ (Poletto \& Kopp 1986; Forbes \& Lin 2000), i.e.,

$$
E_{c}=v_{\mathrm{fp}} B_{\mathrm{ph}} .
$$


where $E_{c}$ is the convective electric field at the magnetic reconnection site, $v_{\mathrm{fp}}$ the observed speed of the apparent HXR footpoint or $\mathrm{H} \alpha / \mathrm{UV}$ flare ribbon and $B_{\mathrm{ph}}$ the vertical component of the photospheric magnetic field. Moreover the energy release rate equals the Poynting flux into the current sheet (e.g., Lee et al. 2006). Estimates of the flux can be obtained from the motions of footpoints and the line-of-sight magnetic field strength at the flare footpoints (e.g., Isobe et al. 2002; Asai et al. 2004a; Temmer et al. 2007).

In the impulsive phase, which observationally is far from two-dimensional, the relationship between footpoint motion, magnetic field and flux transfer rate should be preserved (this follows from magnetic flux conservation) but the coronal reconnection electric field is not so readily obtained. Nor, in an environment of strong twist and shear, will there be a straightforward relationship between $\dot{\Phi}$, the Poynting flux and the energy release rate. We note that a study with a prescribed 3-D coronal field (Hesse et al. 2005) showed a relationship between the instantaneous reconnected magnetic flux at a field line and the "field-lineintegrated" parallel electric field along that field line, suggesting that the endpoints of field lines with high values of this electric field correspond to locations of chromospheric excitation. Although the relationship between $\dot{\Phi}$ and the parallel field is determined by the coronal magnetic configuration, which is not generally known, this analytic work can give us some confidence that $\dot{\Phi}$ calculated from footpoint motions is a meaningful quantity, also in the impulsive phase.

Observationally there are interesting correlations between $\dot{\Phi}$ and properties of the footpoint radiation. Several studies reveal correlations between the HXR flux evolution and the derived reconnection quantities $E_{c}$ and $\dot{\Phi}$, and also with the speed of the footpoint separation or flare loop growth (Qiu et al. 2002; Fletcher \& Hudson 2002; Krucker et al. 2003; Asai et al. 2004a; Liu et al. 2004; Krucker et al. 2005; Veronig et al. 2006; Saba et al. 2006; Miklenic et al. 2007; Temmer et al. 2007). Figure 3.12 shows the result for SOL2003-1029T20:49 (X10.0), with derived $v_{\mathrm{fp}}, v_{\mathrm{fp}} B_{\mathrm{ph}}$ and $v_{\mathrm{fp}} B_{\mathrm{ph}}^{2}$ curves correlated with the RHESSI HXR flux (Krucker et al. 2005). In this flare the HXR flux exponentially correlates with the magnetic field strength at the footpoints, which may scale with the field strength in the coronal reconnection region (Liu et al. 2009b). Figure 3.13 shows the relationship in time of $\dot{\Phi}(t)$ derived from SOL2003-11-18T08:31 (M3.9). Each of the strongest three RHESSI HXR peaks is well reflected in the derived $\dot{\Phi}(t)$ time profiles but shifted in time by $1-2$ min (for a discussion of this effect see Miklenic et al. 2007). The correlation in time and space between locations of high $v_{\mathrm{fp}} B_{\mathrm{ph}}$ and footpoint intensity has also been demonstrated in detail using TRACE UV footpoints (Fletcher 2009).

Interestingly, in the rare example of the HXR ribbon flare SOL2005-05-13T16:57 (M8.0), which has at first glance quasi-2-D properties, there is a better correlation between the HXR intensity and the derived local magnetic reconnection rate and energy release rate when there are only a few isolated HXR footpoints, than when the ribbon-like HXR emission appears (Jing et al. 2007; Liu et al. 2008a).

Further substantial progress in determining the reconnected flux and Poynting flux will be extremely difficult. It will require not only the measurement of the chromospheric vector magnetic field (to assess time-dependent field perturbations at the boundaries) but also microwave observations of gyroresonance emission leading to improved knowledge of the coronal magnetic field. Using multi-frequency microwave observations, such as are planned with FASR (Bastian 2003), isosurfaces of magnetic field strength can be computed. Coupled with plasma flow information from EUV spectrscopic diagnostics, "before and after" changes of the field around a flare would give an independent view of the energy extracted from the field, as well as some information about how it moves through the configuration. Direct observations of the coronal magnetic field at the limb using infrared Zeeman splitting 


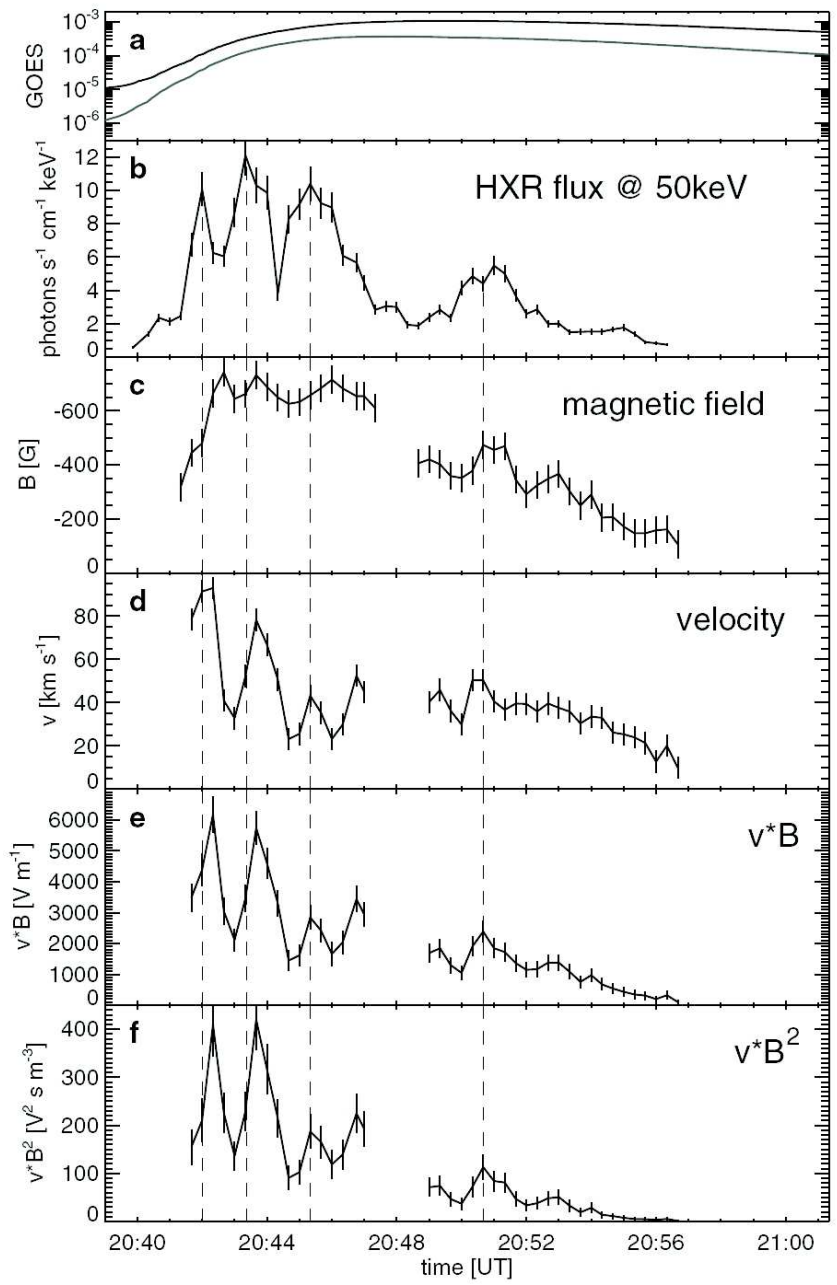

Fig. 3.12 Magnetic reconnection analysis of SOL2003-10-29T20:49 (X10.0). Time series of: (a) GOES SXR flux, (b) RHESSI HXR flux at $50 \mathrm{keV}$ of the Eastern footpoint source, (c) photospheric magnetic field $B$ at the instantaneous footpoint location, (d) velocity $v$ of the HXR footpoint source, (e) product $v B$ (magnitude of convective electric field $E_{c}$ ), (f) product $v B^{2}$ (measure of the energy release rate $\dot{W}$ ) (from Krucker et al. 2005).

(Lin et al. 2000) are proceeding now with the CoMP instrument (Tomczyk et al. 2008). The Hanle effect in the UV (Raouafi et al. 2009) is also utilized.

\subsection{Excitation of the deep atmosphere}

As is well known, the first recorded observation of a solar flare (Carrington 1859) was in the optical or "white light" wavelength range. Since such a flare is visible over and above the bright photospheric radiation $\left(6.27 \times 10^{10} \mathrm{erg} \mathrm{cm}^{-2} \mathrm{~s}^{-1}\right.$ in the quiet Sun), roughly doubling it, this emission is a significant component in the flare energy budget. A small number of 


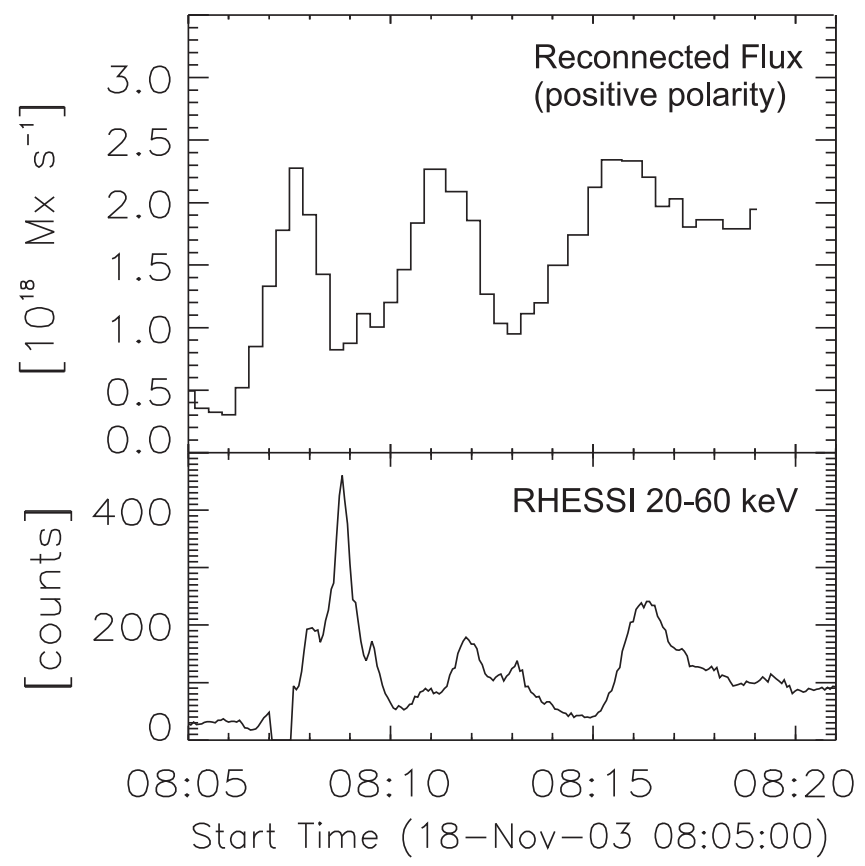

Fig. 3.13 Magnetic flux change rate $\dot{\varphi}(t)$ together with the RHESSI 20-60 keV HXR flux for SOL2003-1118T08:31 (M3.9). Adapted from Miklenic et al. (2007).

direct measurements of the flare total irradiance now exist for large flares (Woods et al. 2006; Kretzschmar 2008), in which the total radiant energy of the flare is measured to be a few times $10^{31}$ to $10^{32} \mathrm{erg}$. White-light emission can also be present in relatively weak flares, down to low GOES C class (Matthews et al. 2003; Hudson et al. 2006; Jess et al. 2008).

The generation of flare optical radiation (IR/visual/UV continuum) is not yet well explained, and it may be that there are different processes operating in different flares. Where spectroscopic observations are available, observed white-light flares have been split into two types (Machado et al. 1986; Neidig 1989). Type I flares 12 show intense and broad Balmer lines and Balmer and Paschen edges (resulting from recombination), and are thought to occur in a heated chromosphere. Type II flares, much less frequent, do not show these features and may arise from enhanced $H^{-}$continuum. The location at which the Type II flare radiation is produced is not known. Generally it is hard to see how the deep layers of the photosphere could be directly excited by electron beams without requiring rather unreasonable electron energy budgets Aboudarham \& Henoux 1986). Excitation by proton beams with energies of 10-20 MeV has also been proposed (e.g., Śvestka 1970; Machado et al. 1978), since protons have a greater penetration depth than electrons of the same velocity. A small velocity dispersion is required to focus the energy deposition over a narrow range of depths, as well as to find agreement with proton fluxes at $\sim 30 \mathrm{MeV}$ implied by $\gamma$-ray observations. Energetic electrons would need to be present in addition, to account for the HXR flux.

12 Type I and Type II as used here should not be confused with the meter-wave radio bursts (e.g., Wild et al. 1963), nor with the spicules (De Pontieu et al. 2007). 
A popular model for the Type I events, which does not require electron beams to reach the deepest layers of the chromosphere, is the "radiative backwarming" model. In this model energy is deposited in the upper chromosphere generating a strong Balmer-Paschen continuum by recombination, which warms lower levels (Metcalf et al. 1990). This idea is close to the original suggestion of Hudson (1972) to bypass the complicated problems of radiative transfer with the "specific ionization" approximation, which implies secondary ionizations. Hudson (1972) and Aboudarham \& Henoux (1986) also note that the non-thermal ionization of hydrogen would also strongly enhance the continua. Non-LTE simulations suggest that a purely chromospheric temperature rise may be insufficient to produce the continuum intensity enhancements seen (Ding \& Fang 1996) and an enhancement near the temperature minimum region may still be necessary. Evidence for the effect of energy deposition was found in the white-light flare SOL2002-09-29T06:39 (M2.6) (Ding et al. 2003; Chen \& Ding 2005). This event had two HXR footpoints, one with weaker HXR emission but stronger whitelight continuum emission, and a relatively weak, centrally-reversed $\mathrm{H} \alpha$ profile (see e.g., Švestka 1966, for a discussion of flares at optical wavelengths). This profile indicates that at the weaker HXR footpoint the atmosphere had not been fully heated, and under such conditions it is possible that an electron beam could effectively penetrate the chromosphere and produce the observed continuum emission via radiative backwarming. By contrast, the local atmosphere at the other footpoint had been appreciably heated, producing a high coronal pressure (Canfield et al. 1984). Electrons would thus be prevented from penetrating into the deeper atmosphere.

Direct evidence for excitation of the deep atmosphere during a flare comes from the flare seismic waves ("sunquakes") first observed by Kosovichev \& Zharkova (1998), and thereafter in several other flares of $\mathrm{M}$ and $\mathrm{X}$ class.
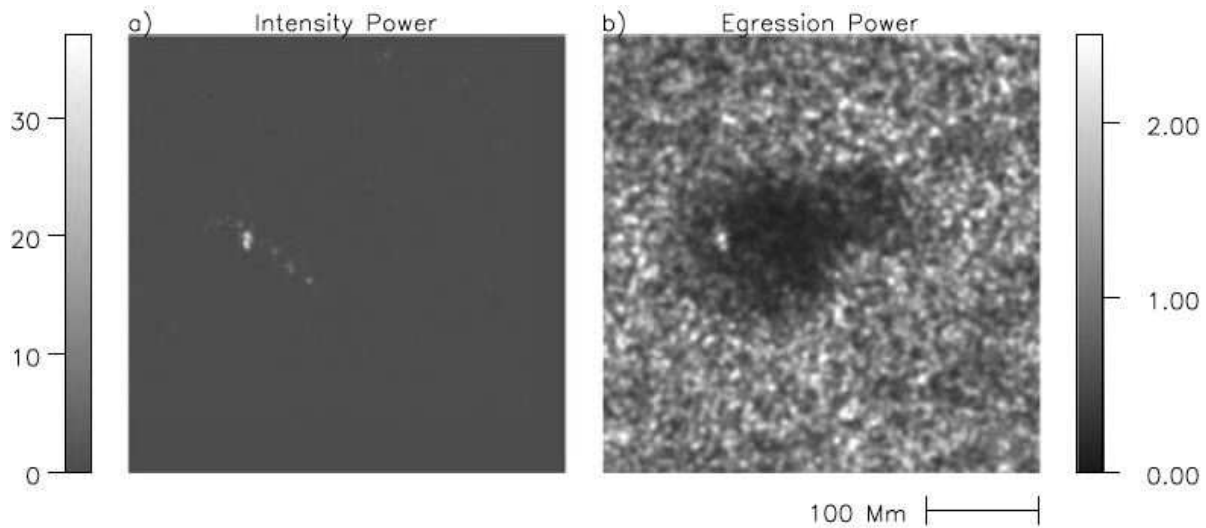

Fig. 3.14 Signatures of SOL2003-10-29T20:49 (X10.0) filtered in the 5-7 mHz band, ie at frequencies above most of the p-mode power. Left: intensity; right: "egression power," showing the source of the seismic waves observed from this flare. The main seismic source is within the area of the large sunspot. From Lindsey \& Donea (2008).

These waves refract through layers deep in the convection zone and appear as surface ripples, traveling at apparent speeds of only some tens of $\mathrm{km} \mathrm{s}^{-1}$, far from the flare site. Seismic waves have been observed from several flares, but they are still comparatively rare - most large flares do not produce detectable wave amplitudes (Donea \& Lindsey 2005). 


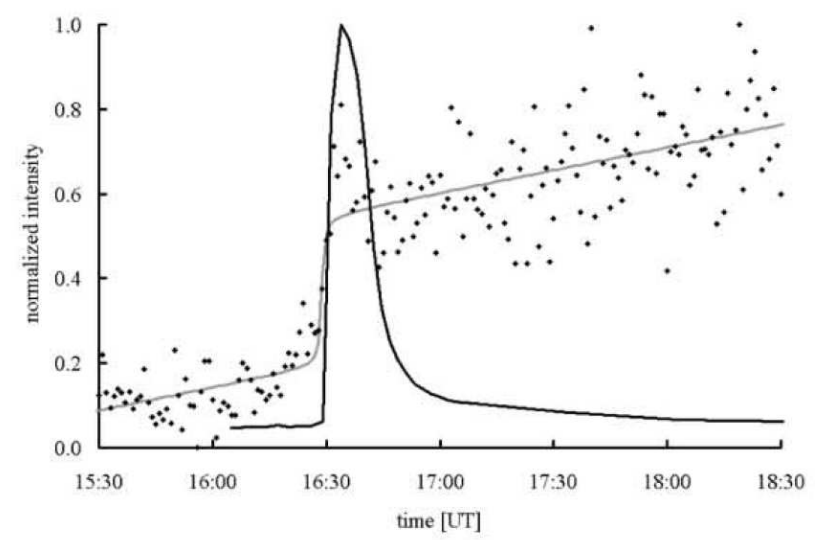

Fig. 3.15 Points and gray line show the normalized time variations of the line-of-sight magnetic field measured by GONG at a point, during SOL2001-08-25T16:45 (X5.3). The line is the TRACE $1600 \AA$ intensity measured co-spatially with the changing magnetic field, to within around $20^{\prime \prime}$. The line-of-sight magnetic field changes abruptly at the time of the flare impulsive phase (from Sudol \& Harvey 2005).

However, the energy required to produce the disturbances is found to be small, on the order of $10^{-4}$ of the total flare energy. The sources of this seismic emission can be located holographically (Figure 3.14), and a large proportion of the seismic sources are located within the penumbrae of sunspots. The sources appear to coincide with the HXR footpoints and whitelight flare kernels (Martínez-Oliveros et al. 2008), and move with them (Kosovichev 2006). They do not appear to be so strongly associated with the $\gamma$-ray sources - i.e., the accelerated ions (Kosovichev 2007) - but the $\gamma$-ray imaging is much inferior to the HXR imaging. Flare seismic waves may be associated with downwards-moving material in the MDI Doppler data. Theory suggests that a shock could be produced by intense heating of the chromosphere by an electron beam, and initially it was proposed that the waves resulted from this shock impacting on the photosphere. However, the momentum required to produce the seismic disturbance is substantially higher than that observed directly in the plasma downflows, and the shock propagation time to the photosphere is inconsistent with observations of the seismic pulse onset versus the HXR peak (Zharkova 2008, and references therein). It is also likely that such shocks would be radiatively damped before reaching the chromosphere (Lindsey \& Donea 2008). Thus another method for delivering momentum into the deep photosphere may be required. Proton beams have been proposed (Zharkova \& Zharkov 2007).

An alternative view is that the seismic waves are launched by a "jerk" of the magnetic field, caused by field re-organization in the corona, imparting momentum to the ions and collisionally-coupled neutrals at the photosphere (Hudson et al. 2008). The jerk is the Lorentz force imparted at the photosphere and is thus capable of launching an interior seismic wave. In the standard reconnection flare model, the perturbation producing the jerk would originate in the corona and propagate as a wave into the photosphere (e.g., Fletcher \& Hudson 2008). Initial analyses cast doubt on the viability of this mechanism (Martínez-Oliveros \& Donea 2009), but it is difficult to disentangle this mechanism and the others proposed: the original idea of a hydrodynamic shock (Kostiuk \& Pikelner 1975; Kosovichev \& Zharkova 1998), and the more recent discussion of direct photospheric heating, e.g., through radiative backwarming (e.g., Lindsey \& Donea 2008). 
The Lorentz-force jerk is consistent with the non-reversible magnetic field changes in the line-of-sight magnetic field observed in many flares. Early observations showed permanent changes in the vector field around the magnetic neutral line (Wang et al. 1994), in one case the observations being separated by only a few minutes before and after the flare. Similar observations for a flare on the limb found variations in the line-of-sight magnetic field (i.e., the component tangential to the photosphere for a limb flare), the importance of this being that the line-of-sight component does not suffer from the $180^{\circ}$ directional ambiguity of the vector field (Cameron \& Sammis 1999). Further work on irreversible changes to the line-of sight magnetic field followed using SOHO/MDI (Kosovichev 2006) and they have now been confirmed to occur in all large flares, usually close in time to the flare impulsive phase (Sudol \& Harvey 2005), and close spatially to the HXR footpoint sources. Figure 3.15 shows an example of such field changes, the typical magnitude of of which is 100-200 G (or on the order of $10 \%$ of the photospheric field in the region). In many cases, the field changes are also associated with visible evolution in the sunspot, particularly to a disappearance of a part of the penumbra (Anwar et al. 1993; Wang et al. 2004; Liu et al. 2005).

Changes of this magnitude at the photosphere imply that the overlying field undergoing rearrangement - presumably in the low corona - must be strong, and energy transported by magnetic disturbances propagating through the chromosphere to the photosphere may be an important component in the flare chromosphere energization (Emslie \& Sturrock 1982; Fletcher \& Hudson 2008). An interesting aspect of such observations is that many flares show an increase in the observational shear along the magnetic polarity inversion line (Wang et al. 1994), counter to what would be expected in a scenario in which the active region free energy should decrease to power the flare. However, Hinode/SOT observations of a flare show that below about $8000 \mathrm{~km}$ above the photosphere the shear increases after a flare, whereas above this altitude it decreases (Jing et al. 2008). It is possible that the shear increase close to the polarity inversion line is associated with flux emergence, as part of the ongoing build-up of magnetic free energy in a repeatedly flaring region.

\subsection{Chromospheric Evaporation}

The arcades of loops characteristic of the gradual phase of solar flares are filled with hot, dense plasma, usually interpreted as chromospheric plasma which expands to a new equilibrium following chromospheric heating in the impulsive phase. This process is termed chromospheric evaporation. It has been studied for almost 40 years, since it was first proposed to explain the delay between the peaks of SXR (gradual) and microwave (impulsive) emissions (Neupert 1968). Latterly, the Neupert effect usually refers to the oftenobserved relationship between time-integrated HXR flux and the SXR flux. The Neupert effect gives indirect evidence for chromospheric evaporation; more direct support comes from observations of blueshifted emission of high-temperature plasma, often correlated with impulsive HXR bursts as discussed further below. Early on, spatially-unresolved 300$400 \mathrm{~km} \mathrm{~s}^{-1}$ upflows in resonance lines of $\mathrm{Ca}$ XIX and Fe XXV were observed using instruments on board the Solar Maximum Mission (Doschek et al. 1980; Antonucci \& Dennis 1983; Zarro et al. 1988), and since then confirmed in spatially-resolved observations with SOHO/CDS 13 (e.g., Czaykowska et al. 1999; Teriaca et al. 2003; Brosius \& Phillips 2004; Del Zanna et al. 2006) and more recently Hinode/EIS (Milligan 2008). It has also been suggested that the heating of quiescent active-region loops is not actually "coronal heating" at

13 Coronal Diagnostic Spectrometer. 
all, but happens in the chromosphere and stems from the principles of flare-induced chromospheric evaporation (Aschwanden et al. 2007).

SXR spectra of flares often show a dominant stationary component as well as the upflow. This is a puzzle for evaporation theory in a single loop excitation, but it could be explained by a filamentary structure, in which many sub-resolution magnetic loops are activated in succession, each having such a small cross-section that it produces undetectably small amounts of emission. Emission would then be detected only after some time, when a number of these loops are emitting together, and the evaporated plasma in each has come to rest at the looptop (Doschek \& Warren 2005). It has also been argued that hot dense plasma exists in the flare corona in advance of the flare impulsive phase (Feldman 1990; Caspi \& Lin 2010). It is usually assumed that the hot emission comes from the flare corona, but recently, stationary Fe XXIV emission has been detected at loop footpoints (Milligan \& Dennis 2009). There are also observations which suggest that the upflows do not reach high into the corona, and that the coronal density increase occurs as a result of compression (Feldman et al. 2004; Caspi \& Lin 2010). An adiabatic compression would in fact create a negative microwave flare in the free-free continuum because the free-free emissivity scales as $T^{-0.5}$. On the contrary, the observations show a good correlation between the radio and X-ray continuum emission measures, so a negative flare is contrary to the observations (e.g., Kundu 1965; Hudson \& Ohki 1972); see White et al. (in this volume) for more detail. Note that negative microwave bursts do occur, but they can be explained by intervening absorptions (Covington 1973).

Early in the flare the chromosphere is heated rapidly and impulsively, primarily by energetic electrons which lose energy collisionally in the chromosphere. Thermal conduction from the corona may also play a role in heating the chromospheric plasma, particularly in pre-impulsive (Battaglia et al. 2009) or gradual (e.g., Zarro \& Lemen 1988) phases. Liu et al. (2009c) also suggest that conduction may play an important role in the impulsive phase for a flare with a substantial low-energy component. The heated atmosphere can radiate or conduct away the energy, and can also expand upwards and downwards. Whether this evaporation is gentle or explosive depends on the energy deposition rate by accelerated electrons as treated in the 1-D radiation hydrodynamics calculations (Fisher et al. 1985a b c; Abbett \& Hawley 1999). For energy input rates of less than $\sim 3 \times 10^{10} \mathrm{erg} \mathrm{cm}^{-2} \mathrm{~s}^{-1}$, theory suggests that the evaporation is gentle, with upward plasma flows at several tens of kilometers per second. Gentle evaporation can also be conductively driven. At high non-thermal electron rates $\left(>3 \times 10^{10} \mathrm{erg} \mathrm{cm}^{-2} \mathrm{~s}^{-1}\right)$, the chromosphere is unable to radiate at a sufficient rate and consequently expands rapidly. This condition is met when the heating time-scale is less than the hydrodynamic expansion time-scale:

$$
\frac{3 k T}{Q}>\frac{L_{0}}{c_{s}}
$$

where $Q$ is the flare heating rate per particle, $T$ is the final temperature of the heated plasma, $c_{s}$ is the corresponding sound speed, and $L_{0}$ is the length-scale of the flaring region. If this condition holds, the heated chromospheric plasma expands upward at hundreds of $\mathrm{km} \mathrm{s}^{-1}$ in a process known as "explosive" evaporation. The overpressure of the flare plasma relative to the underlying chromosphere causes cooler, more dense material to recoil downward at tens of $\mathrm{km} \mathrm{s}^{-1}$ (known as "chromospheric condensation"). In addition to the magnitude of the energy deposition rate, Fisher et al. (1985a) also stipulate that the direction of flows in the transition region/upper chromosphere determines whether the evaporation is gentle or explosive. 

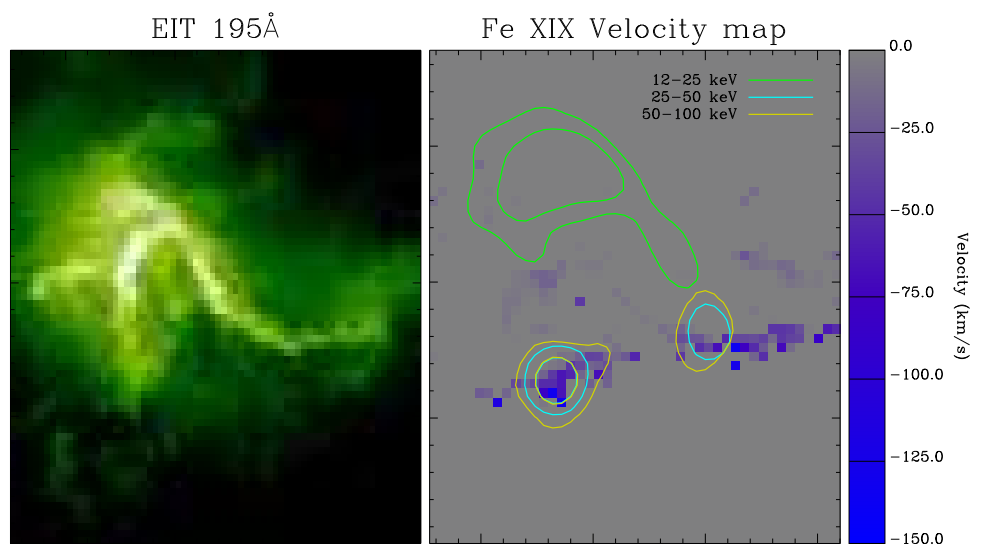

Fig. 3.16 Left: EIT image of a flare observed by SOHO/EIT showing a loop-like structure and possibly an extended EUV ribbon to the west. Right: the event was also observed by RHESSI and the SOHO Coronal Diagnostic Spectrometer. The RHESSI high energy footpoints (yellow contours) coincide with regions of upflow in Fe XIX of formation temperature $\left(\log \mathrm{T}_{\mathrm{e}}=6.9\right)$, with speeds of up to $150 \mathrm{~km}^{-1} \mathrm{~s}^{-1}$. This provides evidence for explosive evaporation driven by electron heating.

Explosive Evaporation: Spatially-resolved spectroscopic EUV observations of explosive upflows during the impulsive phase of a flare are relatively rare, since they require the spectrometer slit to be located at the flare footpoints exactly at the time of strong energy deposition. However, this was managed in SOL2003-06-10T14:36 (M2.2). SOHO/CDS was used to detect simultaneous strongly blueshifted $\left(\sim 250 \mathrm{~km} \mathrm{~s}^{-1}\right)$ Fe XIX emission (peak formation temperature $\log \mathrm{T}_{\mathrm{e}}=6.9$; Mazzotta et al. 1998) from footpoints, supportive of explosive evaporation, along with weakly redshifted He $\mathrm{I}\left(\log \mathrm{T}_{\mathrm{e}}=4.5\right)$ and $\mathrm{O} \mathrm{V}\left(\log \mathrm{T}_{\mathrm{e}}=5.4\right)$ emission (Milligan et al. 2006a). Coordinated RHESSI imaging confirmed that the origin of these flows was at the flare HXR footpoints (see, for example, Fig 3.16). The combination of images and spectra from RHESSI also allows estimates to be made of the energy flux contained in the non-thermal electrons (in erg $\mathrm{cm}^{-2} \mathrm{~s}^{-1}$ ) in order to make a direct comparison with the predictions of theory, under the assumption of the collisional thick-target model. Subject to uncertainties in the area of the unresolved HXR footpoints, this event revealed an electron energy flux greater than $4 \times 10^{10} \mathrm{erg} \mathrm{cm}^{-2} \mathrm{~s}^{-1}$ in agreement with the predictions of Fisher et al. (1985a).

Other observations without HXRs have instead used the time derivative of the GOES flux (assuming the Neupert effect) to identify the impulsive phase (Teriaca et al. 2006; Del Zanna et al. 2006). Spectroscopy from CDS at high time cadence (10 s) of a "flare-like transient" has also been obtained (Brosius \& Holman 2007). All authors report line profiles in hot lines (e.g., Si XII with peak formation temperature $\log \mathrm{T}_{\mathrm{e}}=6.3$, Fe XVI at $\log \mathrm{T}_{\mathrm{e}}=6.4$ and $\mathrm{Fe} X I X$ at $\log \mathrm{T}_{\mathrm{e}}=6.9$ ) consistent with upflows on the order of $100-200 \mathrm{~km} \mathrm{~s}^{-1}$. In the transition lines of $\mathrm{O} v$ at $\log \mathrm{T}_{\mathrm{e}}=5.4$ and $\mathrm{He} \mathrm{I}$ at $\log \mathrm{T}_{\mathrm{e}}=4.5$ downflows of a few tens of $\mathrm{km} \mathrm{s}^{-1}$ were most commonly observed, though upflows are also reported (Del Zanna et al. 2006). Recent observations also confirm the cospatial downflows in $\mathrm{H} \alpha$ spectra and upflows in Ca XIX (first observed by Wuelser et al. 1994) of a few $\mathrm{km} \mathrm{s}^{-1}$ (Teriaca et al. 2006). These can be used to determine the expected momentum balance between the evaporated and condensing material (Canfield et al. 1987). 


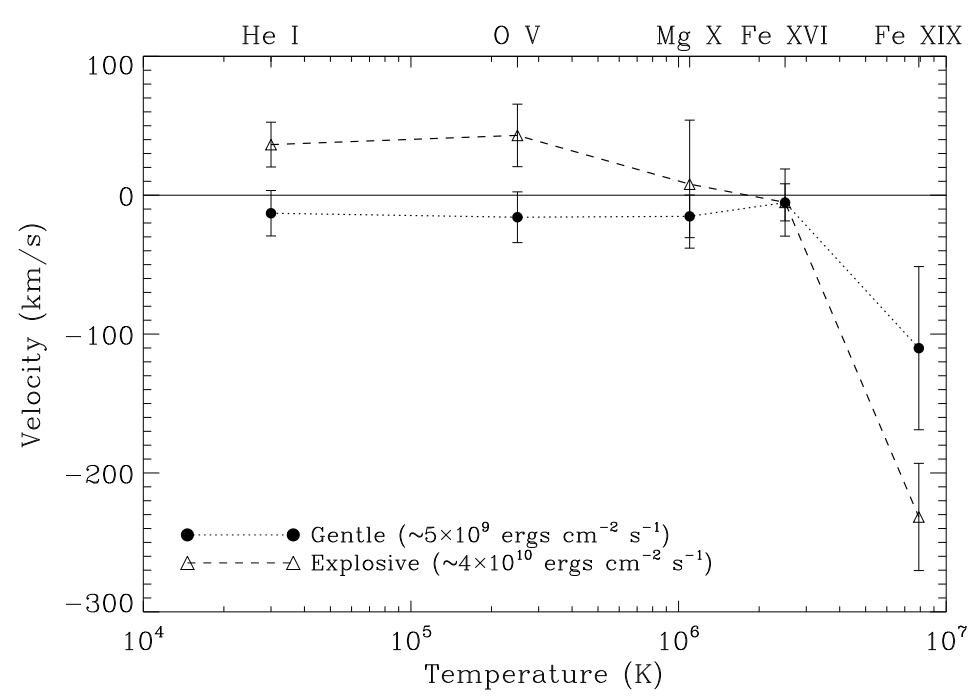

Fig. 3.17 Plasma velocity as a function of temperature for each of the five emission lines observed using CDS during the impulsive phases of two flares, plotted against their characteristic temperatures. Positive velocities indicate downflows (redshifts), while negative values indicate upflows. The data points plotted with filled circles denote the case of gentle evaporation, while the open triangles illustrate the case of explosive evaporation. From Milligan et al. (2006b).

Gentle Evaporation: Plasma flows attributed to gentle evaporation driven by thermal conduction from the overlying hot corona have frequently been observed during the gradual/decay phase of flares, after the non-thermal beam heating has ceased and upflows are sustained by the thermal conduction fronts set up by the steep temperature gradients (e.g., Schmieder et al. 1987; Zarro \& Lemen 1988; Czaykowska et al. 1999, 2001). However, gentle evaporation due to a weak non-thermal electron flux has only recently been observed by SOHO/CDS in conjunction with RHESSI in SOL2002-07-15T11:55 (C9.1) (Milligan et al. $2006 \mathrm{~b}$ ). Doppler shifts of lines formed at a range of temperatures showed upflows of $\lesssim 100$ $\mathrm{km} \mathrm{s}^{-1}$ at all temperatures, consistent with gentle evaporation. The upflow velocity of the Fe XIX material was a factor of two higher when the electron energy flux was an order of magnitude greater (Milligan et al. 2006a) (see Figure 3.17). The absence of any redshifted lines supports the hypothesis that only a large flux of electrons is capable of driving the downflows in the transition region and upper chromosphere associated with explosive evaporation.

The improved spatial, spectral and temporal capabilities of the Extreme Ultraviolet Imaging Spectrometer (EIS) onboard Hinode has also been used to study chromospheric evaporation using emission lines formed over a broad range of temperatures (Milligan \& Dennis 2009). During the impulsive phase of SOL2007-12-14T14:16 (C1.1), blueshifted emission (coincident with RHESSI HXR emission) was observed in six emission lines (Fe XIV-XXIV) formed over the temperature range $\log \mathrm{T}_{\mathrm{e}}=6.3-7.2$. These upflows were found to scale with temperature over the range 8-18 MK, reaching speeds of $>250 \mathrm{~km} \mathrm{~s}^{-1}$ in the Fe XXIV line. This dependence on temperature exists as chromospheric material, heated to a range of different temperatures by a distribution of electron energies, will be subject to different pressure gradients relative to the overlying corona and therefore rise at different rates. A 


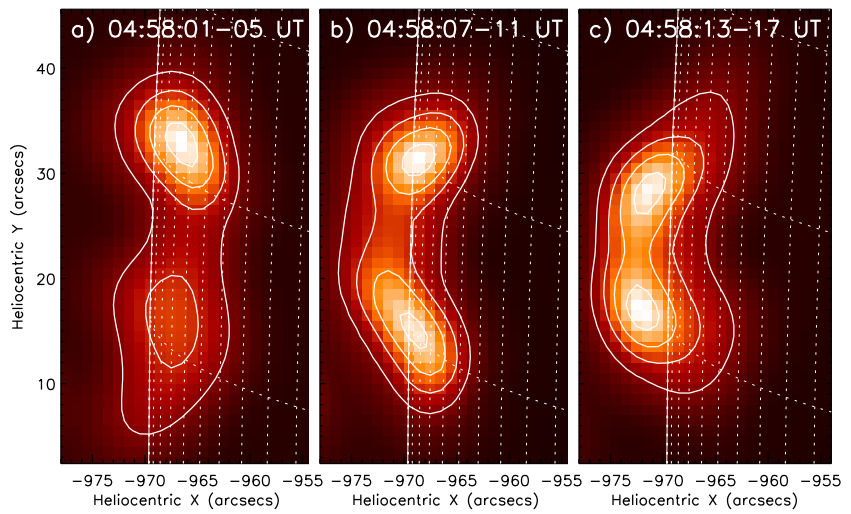

Fig. 3.18 RHESSI hard X-ray images of SOL2003-11-13T05:01 (M1.6) at 12-15 keV integrated over three $4 \mathrm{~s}$ time intervals in sequence. The contours are at $30 \%, 50 \%, 70 \%$ and $90 \%$ of the image maximum. The two sources rapidly move from the footpoints toward the looptop. This is consistent with the flows of hot plasma in chromospheric evaporation, and the Neupert effect is present in this flare as expected (adapted from Liu et al. 2006).

new finding was that cospatial material formed at temperatures from $\log T_{e}=4.7-6.2$ was redshifted by several tens of $\mathrm{km} \mathrm{s}^{-1}$.

At this time the origin of these higher-temperature downflows is unclear and presents a challenge for current evaporation models. They may be related to the spectrum of the electron beam, with a soft spectrum resulting in energy deposition higher in the atmosphere (and downflowing transition-region plasma) and a hard spectrum resulting in energy deposition low in the atmosphere (leading to upflowing transition region plasma). However, a recent model by Liu et al. (2009c) suggests that this may be a result of sustained chromospheric heating, rather than a single heating burst as is used in most modeling.

Imaging of Evaporation: Direct imaging observations of the expected fronts of multimillion K upflowing plasma in the act of filling the loops are rather hard to come by (Doschek et al. 1996). One reported observation with Yohkoh/SXT (Silva et al. 1997) has features suggestive of upwards plasma flows at the rather slow speed of around $60 \mathrm{~km} \mathrm{~s}^{-1}$ in the impulsive phase. Evidence for evaporative upflows has been claimed in RHESSI observations of SOL2003-11-13T05:01 (Liu et al. 2006). This event has a pair of footpoint/loop leg sources in the range 12 to $30 \mathrm{keV}$ (see Figure 3.18), which converge into a single source near the center of the loop at a speed of some hundreds of $\mathrm{km} \mathrm{s}^{-1}$, perhaps as much as $10^{3} \mathrm{~km} \mathrm{~s}^{-1}$. The emission centroids in this event shift systematically toward the footpoints with increasing energies up to $\sim 70 \mathrm{keV}$, and the upward source motion occurs first at low energies and progresses to higher energies. The source motion is thus also consistent with the behavior expected in a coronal thick target (see Section 4.1). This is accompanied by an increase in density at the looptop (see also Jin \& Ding (2008) who observe the appearance of a coronal loop at high energies).

Finally, a recent observation of a weak B1.7-class flare using RHESSI and Hinode/EIS found hot $(\sim 2 \mathrm{MK})$ upflowing plasma at one footpoint, but hot downflowing plasma at the other (Milligan 2008), whereas both theory and previous observations at lower spatial resolution have only shown upflows at this temperature. An interpretation in terms of a 
siphon-like flow is inconsistent with the apparent filling of the flare loop observed with Hinode/XRT.

\section{Coronal Sources}

The presence of coronal hard X-ray sources was first inferred in disk-occulted events (flares with HXR footpoints behind the solar limb) using data from HXR spectrometers on the OSO-5 and OSO-7 satellites. (Frost \& Dennis 1971; Hudson 1978) The radial height above the likely active regions in these cases could be estimated at more than $2 \times 10^{4} \mathrm{~km}$. This placed them above the mean heights of flare SXR sources (Catalano \& van Allen 1973; van Beek et al. 1981). Similar HXR source altitudes were inferred using observations from multiple spacecraft (Kane et al. 1979a). The earliest HXR imaging (from Hinotori) directly showed coronal source energies up to at least $25 \mathrm{keV}$ (Takakura et al. 1983).

The launch of the Yohkoh satellite led to several further reports of coronal emission. Observations of an impulsive, and by implication, non-thermal coronal component, with energies up to the 33-53 keV band, were first made by using Yohkoh/HXT (Masuda et al. 1994). More Yohkoh/HXT coronal sources were subsequently found, though few with quite the same remarkable properties as the "Masuda flare." HXR coronal sources were demonstrated to exhibit both gradual and impulsive HXR characters, with the impulsive spikes being more energetic and having harder spectra (Tomczak 2001).

The RHESSI observations have substantially added to the literature on coronal HXR sources, and it is one area in which RHESSI has made a tremendous impact. This body of new observational work has recently been summarized, along with an overview of the theoretical ideas, by Krucker et al. (2008a). This paper should be consulted for more detail on this rich and relatively new field than can be provided below.

\subsection{Thick-target looptop sources}

Coronal densities are generally too low to produce HXR emission efficiently, which is why footpoint sources normally dominate the images. The observation of the Masuda source inspired the suggestion that it could be explained by a partially thick coronal target Wheatland \& Melrose 1995). Subsequent observations with RHESSI (Veronig \& Brown 2004) uncovered a new type of coronal HXR emission embedded in coronal loops, rather than "above the loop top" as in the Masuda source. Several events could be interpreted as "coronal thick target" sources (Figure 4.1), identifiable by their lack of compact footpoint HXR sources. In the first case, SOL2002-04-15T00:15 (M3.7), the spectrum observed with RHESSI was rather steep $(\gamma \geq$ 6 ) and the column density $\mathrm{N}_{\text {loop }}$, estimated using GOES, was high $\left(N_{\text {loop }} \geq 10^{20} \mathrm{~cm}^{-2}\right)$, leading to the interpretation that plasma in the corona was dense enough to act as a thick target. This can happen when the stopping energy $E_{\text {loop }}=\sqrt{3 K N} \approx 8.8 \sqrt{N_{19}} \mathrm{keV}$ is greater than the energy of an electron, where $\mathrm{N}_{19}$ is the column density in $10^{19} \mathrm{~cm}^{2}$. A $25 \mathrm{keV}$ electron has a range (stopping column density) of about $10^{20} \mathrm{~cm}^{-2}$ (Brown 1973). In the Veronig-Brown flare such a column density appears already to have been present within the loop at the onset of the flare, and during the flare it increased to several times $10^{20} \mathrm{~cm}^{-2}$ which allowed electrons up to $50 \mathrm{keV}$ to be fully stopped.

A high pre-flare coronal density is puzzling, because if the material is at coronal temperatures and static, the resulting high equilibrium pressure will make it a bright $\mathrm{X}$-ray 


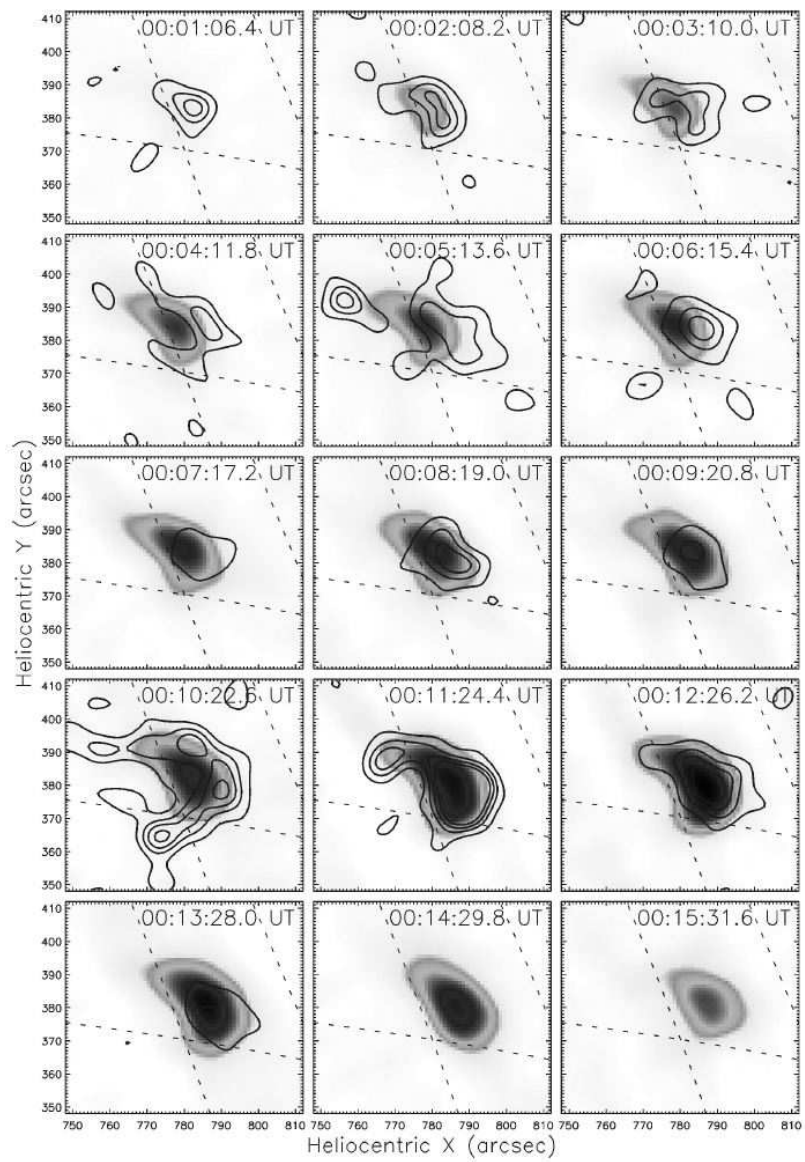

Fig. 4.1 RHESSI images of a thick-target looptop source in SOL2002-04-15T00:15 (M3.7) (Veronig \& Brown 2004): images 6-12 keV, contours $25-50 \mathrm{keV}$ with minimum level 0.17 times the maximum in each frame. The HXR contours show little evidence for footpoint brightening.

source. This consideration would not apply to a prominence or a loop structure at intermediate temperatures. Having a high density at the start of a flare is also a difficult problem for the standard flare model, which envisions the opening of the field prior to the reconnection. An earlier flare in the same region could create an enhanced coronal column density by evaporation in a loop that subsequently flares again (Veronig et al. 2005), or, similarly, slow pre-flare heating could lead to evaporation into a system which then becomes unstable. However, at least in the standard 2-D scenario, electrons would propagate down a different set of field lines from the closed, post-reconnection loops onto which the plasma has already evaporated, precluding the scenario in which energetic electrons are accelerated onto dense loops as a result of subsequent reconnection. A more complicated magnetic geometry or a less direct link between reconnection and acceleration is needed.

The SOL2002-04-15T00:15 (M3.7) "coronal thick target" event could also be observed via Nobeyama microwave imaging at $17 \mathrm{GHz}$. These data include a circularly polarized component (Veronig et al.2005; Bone et al. 2007), establishing the presence of non-thermal 


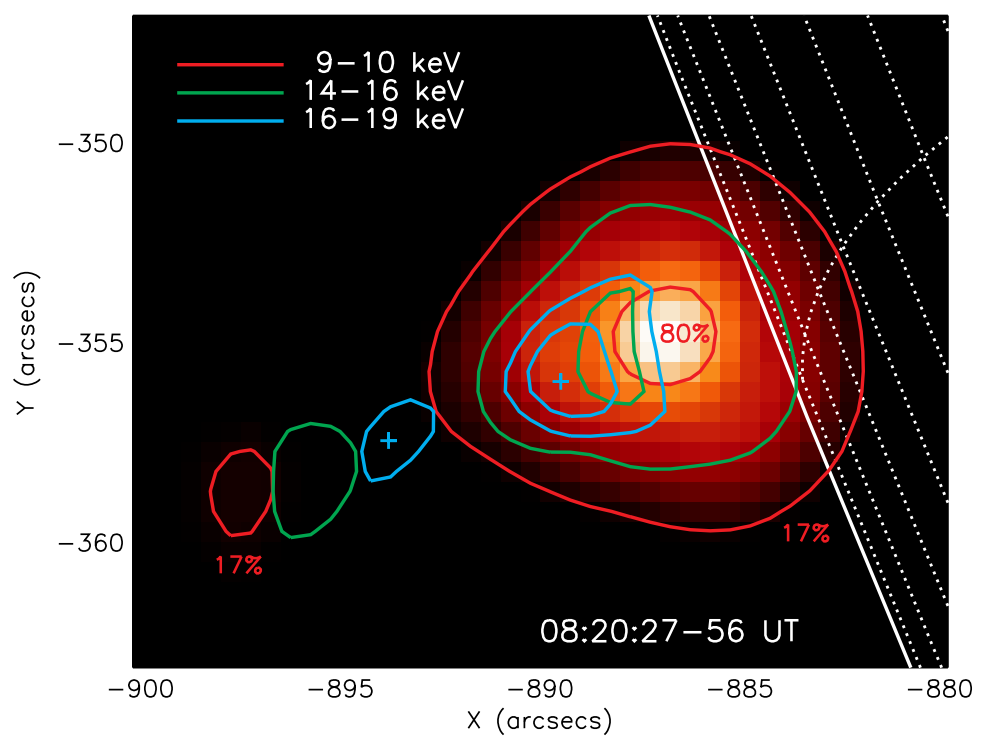

Fig. 4.2 RHESSI soft X-ray observations (Liu et al. 2008b) of a double coronal SXR configuration in SOL2002-04-30T08:22 (M1.3) suggesting the presence of a current sheet, as originally discovered by Sui \& Holman (2003). The separation between coronal and loop-top sources decreases as a function of photon energy. The separation between the $16-19 \mathrm{keV}$ source centroids (+ signs) was found to be $4.6 \pm 0.3^{\prime \prime}$ in this case.

electrons, visible in the whole loop through unpolarized thermal emission and consistent with the high density needed for the coronal thick target (Bone et al. 2007). A rather nonstandard explanation is that the previously flaring dense loops could become unstable due to a high-beta instability resulting in a second flare (Shibasaki 2001).

The idea of a coronal thick target might extend to a seemingly separate class of events, namely the "soft-hard-harder" coronal HXR sources discussed below in Section 4.5. This possibility might require the stable trapping of high-energy electrons in a theoretically unstable loss-cone distribution (Wentzel 1976). Other than high density and magnetic trapping, turbulence or plasma waves (e.g., Benz 1977; Miller et al. 1996; Petrosian \& Liu 2004), generated as a consequence of magnetic reconnection, provide an alternative mechanism that could accelerate electrons and, at the same time, confine them to the region near the looptop.

4.2 X-ray observations suggesting coronal current sheets

RHESSI observations revealed the existence of double coronal X-ray sources, interpreted in terms of the current sheet expected in the large-scale reconnection model.

In a series of flares that occurred during April 2002, a coronal X-ray source was observed above the flare loops and was detectable to about $20 \mathrm{keV}$ (Sui \& Holman 2003; Sui et al. 2004). This source was initially stationary before moving outwards at around $300 \mathrm{~km} \mathrm{~s}^{-1}$ (Sui \& Holman 2003). RHESSI images typically show a height dependence on energy in the flare loops, with the higher energy X-ray sources located above the lower energy sources, whereas here the lower-energy sources are located above the higher-energy sources (e.g., 


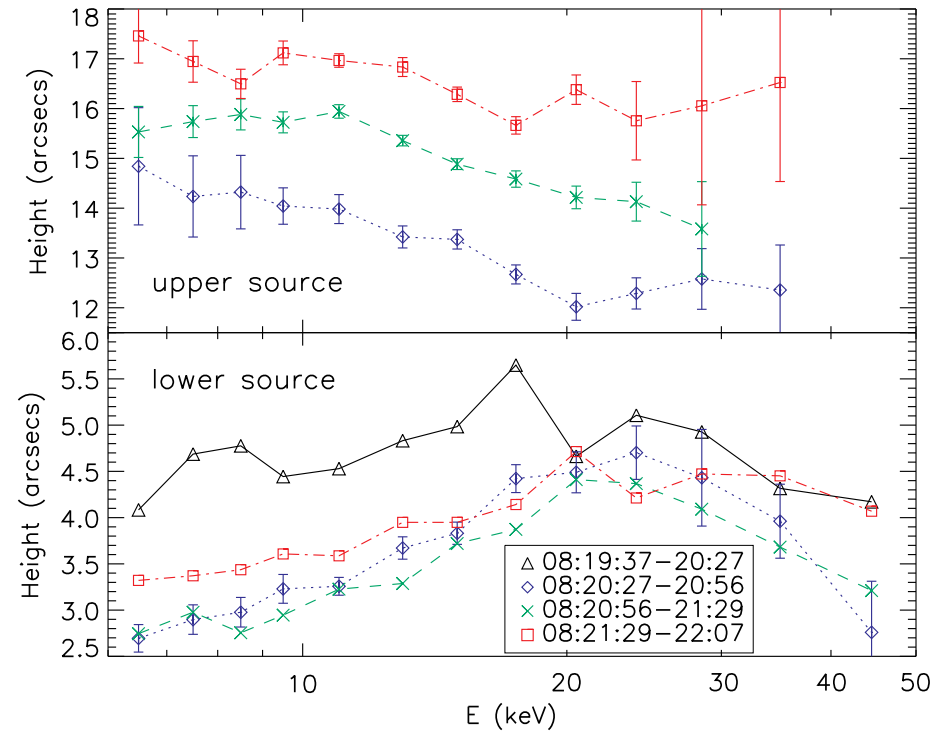

Fig. 4.3 Height above the limb of the centroids for the upper and lower coronal sources in SOL2002-0430T08:22 (M1.3) plotted as a function of energy for four consecutive time intervals. Note the reversal of the trend from low-energy thermal to high-energy non-thermal regime (from Liu et al. 2008b).

Figure 4.2). These observations matched the theoretical expectations for signatures of a current sheet formed between the top of the flare loops and the coronal source, although the velocity of the coronal source may be somewhat too low to be consistent with Alfvénic ejection speeds from the upper end of a large-scale current sheet (the same comment applies to the speed of supra-arcade downflows). For one of these events EUV spectroscopic observations were available which showed high-speed, high-temperature plasma flows near the inferred current sheet; these observations were interpreted as reconnection outflows (Wang et al. 2007). Similar but less prominent double coronal X-ray sources were reported by Veronig et al. (2006) in SOL2003-11-0T309:55 (X3.9) and by Li \& Gan (2007) in the occulted flare SOL2002-11-02T06:07 (C3.9).

Imaging spectroscopy of a double coronal source was carried out for SOL2002-0430T08:22 (M1.3) (Figure 4.3, see Liu et al. 2004). The HXR footpoints were occulted by the limb, and this facilitated imaging the otherwise relatively faint coronal sources at energies up to $\sim 40 \mathrm{keV}$. The two coronal sources, both visible for about 12 minutes, had similar light curves and power-law spectra above $\sim 20 \mathrm{keV}$, suggesting production by similar populations of non-thermal electrons possibly energized by a common acceleration mechanism. At low energies $(\lesssim 20 \mathrm{keV})$, both sources were dominated by thermal emission, and the lower coronal source had a larger emission measure but a lower temperature, suggesting that the different magnetic connectivity above and below the current sheet could lead to different plasma densities. In addition, the trend of the energy-dependent source structure (Sui \& Holman 2003) visible at thermal energies showed a reversal above $\sim 25 \mathrm{keV}$ (see Figure 4.3, with the two sources being further away from each other at higher, non-thermal energies. A possible explanation is the larger stopping distances, from the acceleration site, of the higher energy electrons. The above two properties were also found in SOL2003-0424T15:53 (C8.2) (Liu et al. 2009; see their Figure 5). 
4.3 Early-phase coronal sources

In its first $\gamma$-ray flare, SOL2002-07-23T00:35 (X4.8), RHESSI also discovered a remarkable new type of coronal HXR source. The observations showed a coronal HXR predecessor to the main impulsive phase of a flare (Lin et al. 2003; Asai et al. 2006). The HXR spectrum of these early coronal sources extends directly down to low energies $(<10 \mathrm{keV})$ with powerlaw indices of around 5, but the spectra also show the characteristic $\mathrm{Fe}$ emission feature at $6.7 \mathrm{keV}$ (e.g., Phillips 2004), establishing that some high-temperature background plasma also exists in (or near) the source (Caspi \& Lin 2010). While the time evolution of the thermal component is gradual, the emission at higher energies shows time variations of tens of seconds' duration suggesting that the two components are produced by different emission mechanisms. X-ray spectral fitting of the high energy component shows that either a nonthermal model (i.e., broken power-law spectrum) or a multi-thermal model (temperatures up to $\sim 100 \mathrm{MK}$ are needed) can represent the spectra well. However the microwave observations (Asai et al. 2006) favor the non-thermal alternative (see White et al. 2011). This interpretation suggests strong coronal magnetic fields (around $200 \mathrm{G}$ ) at relatively high altitudes $\left(>2 \times 10^{4} \mathrm{~km}\right)$.

Despite the ambiguous continuum models, the $\mathrm{Fe}$ and $\mathrm{Fe} / \mathrm{Ni}$ line emission can also be used to constrain the thermal plasma parameters. During the impulsive and decay phases of the flare, the fluxes of the two line complexes and their ratio are correlated with the continuum temperature; by assuming that the same relationship holds during this pre-impulsive phase, the observed line fluxes and ratio (which can be accurately measured by RHESSI) thus provide upper and lower limits on the temperature and emission measure of the thermal plasma. During the peak of the pre-impulsive phase, the line observations constrain the thermal component temperature to be between $\sim 29$ and $\sim 37 \mathrm{MK}$; a cooler component with temperature between $\sim 21$ and $\sim 18 \mathrm{MK}$ (respectively) is also required to fit the SXR spectrum. Consequently, the low-energy cutoff of the non-thermal electrons is at least as low as $\sim 20$ and $\sim 27 \mathrm{keV}$, respectively (Caspi \& Lin 2010).

Strong coronal magnetic fields are also supported by observations of the thermal plasma later in the flare. At the time of peak temperature of SOL2002-07-23T00:35 (X4.8), the ther-

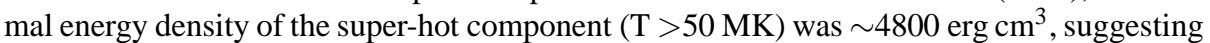
a coronal field strength exceeding $\sim 350 \mathrm{G}$ to contain it. We note that a survey of $37 \mathrm{M}$ - and $\mathrm{X}$-class flares shows that strong coronal fields $(>200 \mathrm{G})$ are required in all X-class flares, which were also invariably super-hot (Caspi 2010) in nature. All three HXR-dominated preimpulsive sources observed by RHESSI were X-class flares, and this suggests an intimate link between the existence of super-hot plasma, strong coronal fields, and the existence of such pre-impulsive HXR sources.

\subsection{High coronal sources}

For flares occurring more than about $20^{\circ}$ behind the solar limb, the occultation should normally be deep enough to hide not only the footpoint sources, but also the main flare loops as well (e.g., Tomczak 2001). This opens the possibility of observing emissions from the high corona ( $200 \mathrm{Mm}$ above flare site), and in fact the early non-imaging observations showed that such events really do happen (Frost \& Dennis 1971; Hudson 1978; Hudson et al. 1982), if rarely. HXR emissions from a flare occulted by $40^{\circ}$ as seen from Earth, corresponding to an occultation height of roughly a third of a solar radius, have been reported (Kane et al. 1992). Despite this large occultation height, HXR emissions were observed up to $80 \mathrm{keV}$, 
with a rather hard spectrum $(\gamma<3.5)$. Another high coronal event observed by Yohkoh Hudson et al. 2001) revealed rapid outward source motions with accompanying microwave emission. The event morphology suggested filament eruption and and CME occurrence. An early HXR stereo observation using multiple spacecraft (Kane et al. 1979a) showed that the HXR emissions from the high corona can occur during the impulsive phase of the flare simultaneously with the HXR footpoint emissions, and the source size might at the same time be large (of order $200^{\prime \prime}$ ).

The RHESSI observations (Krucker et al. 2007) of high coronal events all tend to have similar time profiles. They show a fast rise and a slower exponential decay, as illustrated in Figure 4.4 The exponential decay is surprisingly constant, lasting sometimes several minutes without significant deviation, and the photon spectrum exhibits progressive spectral hardening (hence the "soft-hard-harder" morphology - Cliver et al. 1986; Kiplinger 1995; Grigis \& Benz 2008). The decay suggests that collisional losses - without further acceleration - dominate. Density estimates of the ambient plasma support this; these allow estimates of collisional loss timescales of $25 \mathrm{keV}$ electrons comparable with those measured. While the flare-accelerated electrons in the high corona are only a small fraction $(0.1 \%)$ of the total number of accelerated electrons in the flare (Kane et al. 1992), the relative number of energetic electrons $(>10 \mathrm{keV})$ in the high coronal source may be of order $10 \%$ of the thermal electrons.

\subsection{Gradual late-phase sources}

Gradual late-phase sources are characterized by flat HXR spectra (power-law index $\gamma \approx 2$ ), gradual time profiles, low microwave peak frequencies, anomalously weak SXR emission, and association with coronal radio bursts. The prototype event SOL1969-03-30T02:47 (Frost \& Dennis 1971) occurred in an active region known circumstantially to have been some distance behind the solar limb (Palmer \& Smerd 1972) so that the HXRs visible from the Earth-orbiting $O S O-5$ spacecraft probably originated from relatively high in the corona.

A "soft-hard-harder" pattern of spectral evolution characterizes many long-duration HXR events (Cliver et al. 1986; Kiplinger 1995; Grigis \& Benz 2008). This pattern differs from the otherwise ubiquitous "soft-hard-soft" pattern associated with the impulsive phase (Parks \& Winckler 1969; Hudson \& Fárník 2002; Grigis \& Benz 2004). From a non-imaging perspective, the HXR spectrum of such a source consists of a gradual, continuously hardening component plus a series of spikes with soft-hard-soft evolution. Often these spikes become more gradual as the event develops (Saldanha et al. 2008); see also the early non-imaging observations from the TD-1A spacecraft (Hoyng et al. 1976). Figure 4.5 illustrates this.

The physics of the gradual-phase coronal HXR sources with their "soft-hard-harder" temporal development remains to be worked out. There is every reason at present to suppose that the observed spectral flattenings and exponential-law time decays can be explained by some combination of trapping and collisions, but important theoretical work involving wave-particle interactions, specifically loss-cone instabilities, and large-scale magnetic restructurings remains to be done. A possibly interesting theoretical aspect of these sources is the idea that the non-thermal particles could dominate the background plasma component energetically (e.g., Hudson et al. 2001).

Because of their long duration, a thin-target explanation might be imagined, such that the HXR emission would come predominantly from a coronal trap. However it now appears (Qiu et al. 2004) that these late sources emit hard X-rays mainly from footpoints, at least at energies below $100 \mathrm{keV}$, as with ordinary flare loops. 

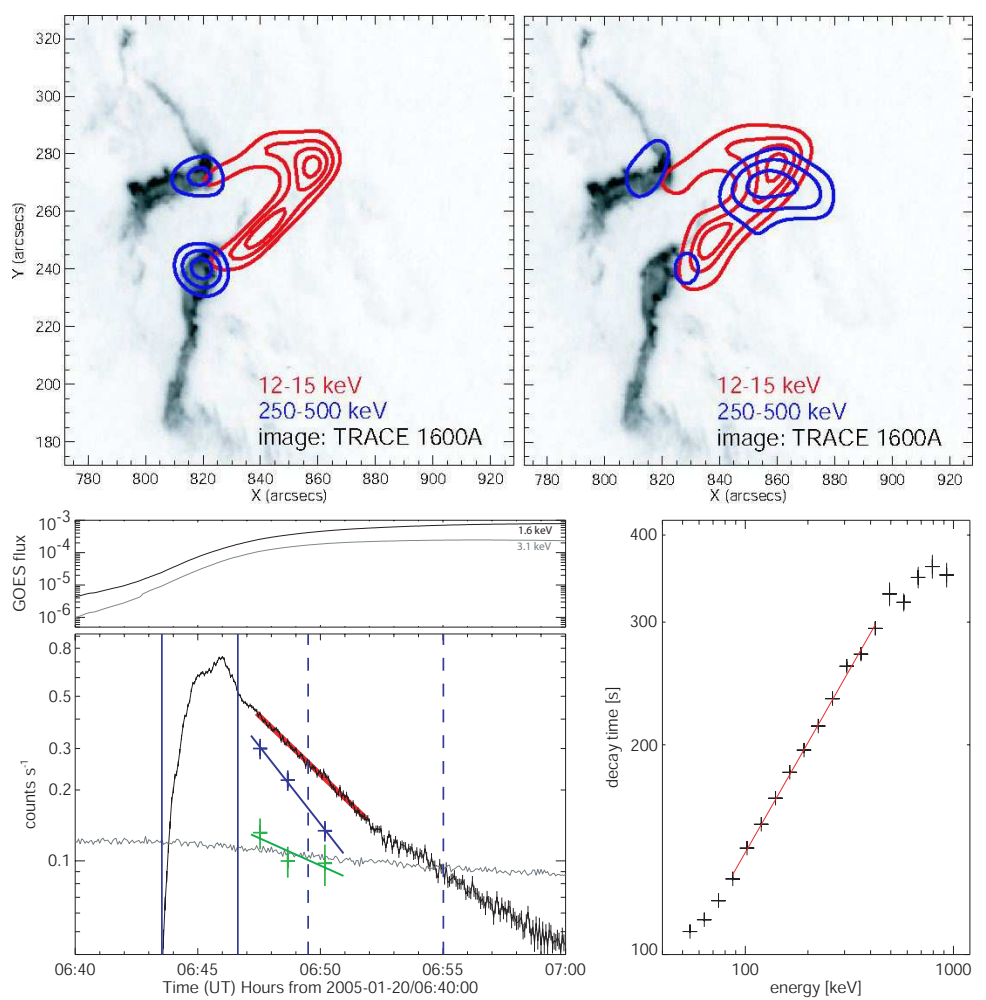

Fig. 4.4 Upper: RHESSI hard X-ray observations at 12-15 keV (red) and $>250 \mathrm{keV}$ (blue) from SOL200501-20T07:01 (X7.1) Krucker et al. 2008a), showing a strong high-energy coronal hard X-ray source. The background TRACE image shows the flare ribbons as observed in the UV by TRACE, and the contour levels are at $30-90 \%$ of the image maxima. Lower: at left, the light curves, with GOES soft X-rays at the top and RHESSI hard X-rays at the bottom. The latter show three points in the time histories of the looptop and footpoint sources at $>250 \mathrm{keV}$, while the full curve shows the total. The footpoints decay more rapidly. The plot at lower right shows the decay time as a function of photon energy.

\subsection{Looptop source motions}

Upwards: A flare arcade gradually develops to larger and larger scales with time, a process which has long been observed in chromospheric emission lines such as $\mathrm{H} \alpha$ and in SXRs. RHESSI observes this phenomenon in a different manner because of its uniquely sensitive imaging spectroscopy in the 3-20 keV range. Figure 4.6 shows this graphically for one of the first X-class long-duration flares observed by RHESSI (Gallagher et al. 2002). The "shrinkage" expected (Švestka et al. 1987; Forbes \& Acton 1996) from the standard reconnection model is clearly visible, in the sense that the higher-energy (higher-temperature) $\mathrm{X}$-ray sources, identifiable with loop tops, lie systematically at higher altitudes than the EUV sources that they presumably evolve into.

Downwards: Observations of hot flare loops made by RHESSI in X-rays show that in many cases the loops contract downward during the early, most explosive part of the flare before the apparent outward expansion is observed. The downward motion of the X-ray loop-top 


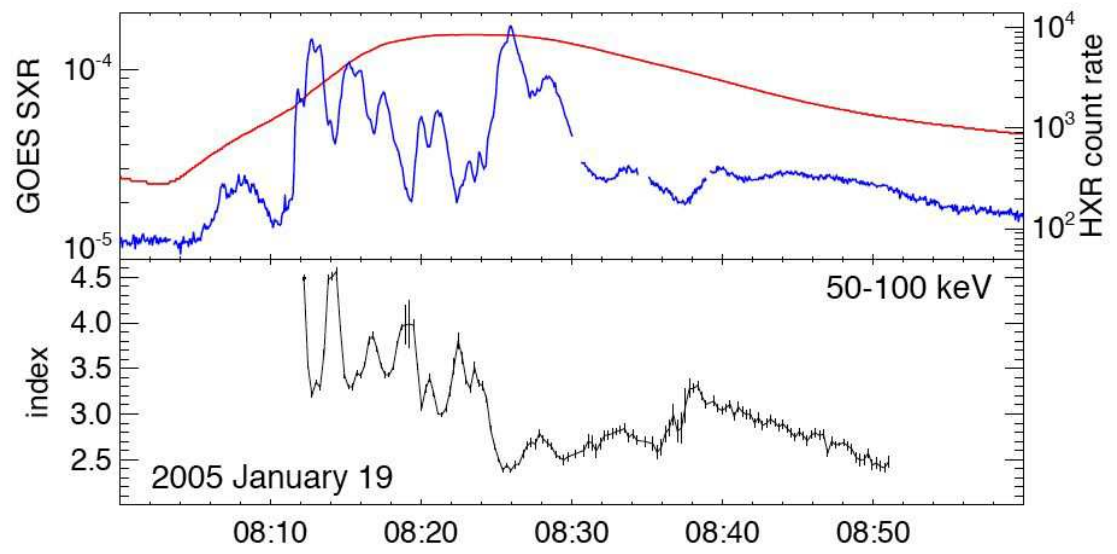

Fig. 4.5 Recent illustration of the soft-hard-harder spectral evolution in the late phase of SOL2005-0119T08:22 (X1.3) (adapted from Saldanha et al. 2008). Note the anticorrelation between HXR flux (upper, blue line; the red line shows the GOES SXR flux) and HXR spectral index (lower) at the beginning of the event, during the more impulsive variations. Towards the end the variability has longer time scales and the spectral index systematically diminishes (gradual hardening).

centroid early in the impulsive phase of solar flares has only recently been recognized. This new observation may have been missed with the Yohkoh observations because of coverage biases induced by the operation of its flare mode, which only initiated hard X-ray spectral observations at a soft X-ray flux level typically corresponding to a low C-class flare. The first reported observation, during the rising phase of SOL2002-04-15T00:15 (M3.7), showed shrinking of the underlying HXR flaring loop at $\sim 9 \mathrm{~km} \mathrm{~s}^{-1}$ (Sui \& Holman 2003). Several further reported events confirm this pattern (e.g., Liu et al. 2004; Sui et al. 2004; Veronig et al. 2006; Joshi et al. 2007).

In all events, the looptop sources of the flares at higher X-ray energy bands were located at higher altitudes and showed higher downward velocities than at lower energies. For example, in SOL2003-11-03T09:55 (X3.9) (Liu et al. 2004; Veronig et al. 2006) the mean downward velocities range from $45 \mathrm{~km} \mathrm{~s}^{-1}$ in the $25-30 \mathrm{keV}$ band to $14 \mathrm{~km} \mathrm{~s}^{-1}$ in the RHESSI 10-15 keV band, and in SXR observations from the GOES Soft X-ray Imager (SXI) the looptop altitude decreased at $12 \mathrm{~km} \mathrm{~s}^{-1}$, in agreement with the general trend (Veronig et al. 2006). This trend of lower speed at lower energies is also carried through the wavelength range to loops that are both shrinking and cooling, visible in EUV and $\mathrm{H} \alpha$ (Vršnak et al. 2006). An interesting phenomenon that follows the energy-dependent looptop source velocity is the anti-correlation between the HXR flux and the separation between emission centroids of the looptop sources at different energies as shown in Figure 4.7 (Liu et al. 2004, $2008 \mathrm{~b}$ ). This has been interpreted as the looptop source being more spatially homogeneous during the HXR peaks, as a consequence of the rapid energy release. It is now known that the early downwards motion of coronal HXR sources is a common characteristic of flares.

The explanation for the converging motion of conjugate footpoints and the simultaneous descending of looptop sources is still in a preliminary stage of development. As a basic consequence of the extraction of excess energy from the magnetic field the contraction of flaring loops at the initial phase of solar flares should occur as an "implosion" (Hudson 2000; Janse \& Low 2007; Liu et al. 2009a), a process consistent with the observations men- 


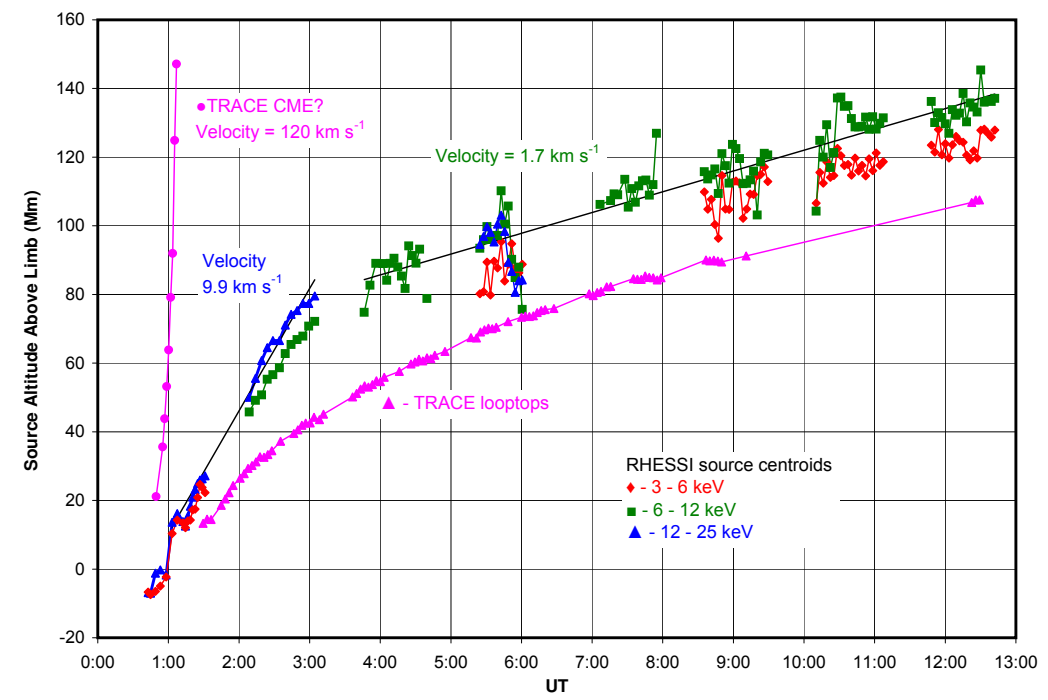

Fig. 4.6 Observations of SOL2002-04-21T01:51 (X1.5), an event at the extreme W limb (adapted from Gallagher et al.2002). The plot shows apparent altitude above the solar limb as a function of time for features seen in RHESSI and TRACE images. The slower motions are related to the growth of the arcade, and the RHESSI data clearly show higher temperatures at larger altitudes. The rapid motions at the beginning are associated with the CME (Gallagher et al. 2003).

tioned in the previous paragraph (see also Section 3.4). In the framework of a reconnection model with sheared magnetic field lines, the contraction might be caused by the relaxation of highly sheared magnetic field after magnetic reconnection. In a force-free arcade in which the magnetic field strength decreases exponentially with height, the dissipation of magnetic energy in a flaring region could lead to a decrease in the scale height of the magnetic field and thus a shortening of the field lines (Ji et al.2007). Therefore, in the initial phase of flares, the contraction caused by the relaxation of highly-sheared core magnetic field may dominate over the apparent expansion of the hot loops which occurs as a result of reconnection taking place at higher and higher altitudes. In such a case it is interesting to note that the remaining shear may indicate stresses in the field remaining even after the flare has occurred.

\section{Flare relationships to coronal mass ejections, dimmings, and particles}

\subsection{Overview}

The large-scale behavior of the solar corona during a flare or CME disruption has many observational consequences. Traditionally the corona itself has only been observable as Thomson-scattered photospheric light, but well above the photosphere so as to avoid its 


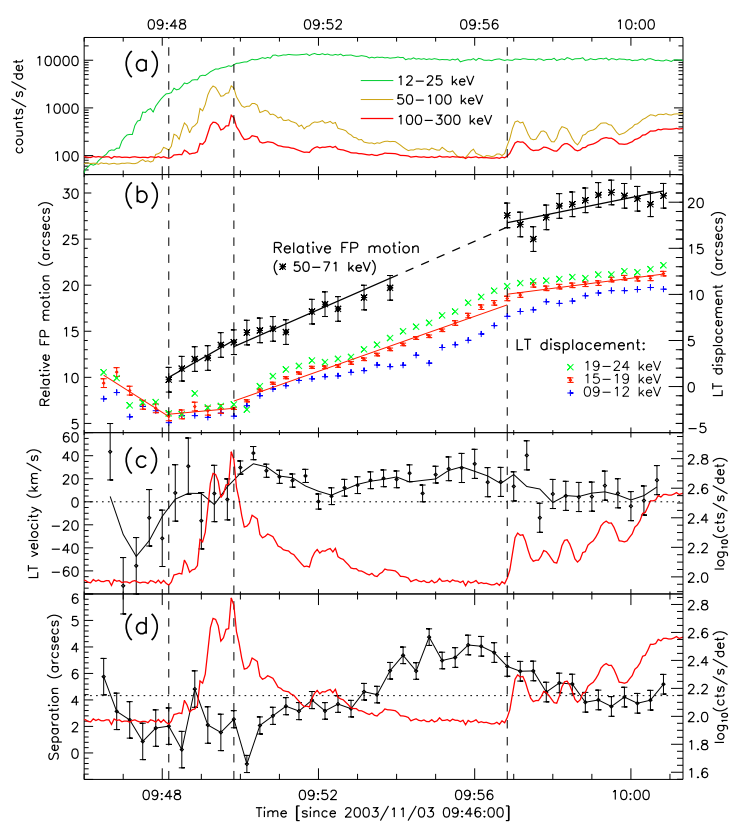

Fig. 4.7 Looptop and footpoint source motions in SOL2003-11-03T09:55 (X3.9) and the energy-dependent source structure. (a) RHESSI light curves. (b) Projected height of the looptop centroid (right scale) and the separation of the two footpoints (left scale), which both increase at comparable speeds. Note the early downward looptop motion. (c) Velocity of the looptop at $15-19 \mathrm{keV}$, with the values smoothed over 1-minute intervals shown as the dark line. The red curve here and in panel $d$ is the logarithm of the $100-300 \mathrm{keV}$ count rate (right scale). (d) Separation of the looptop centroids at $19-24 \mathrm{keV}$ and 9-12 keV, which is in anti-correlation with the HXR count rate (from Liu et al. 2004).

glare. Thus most of the low corona, with high density and strong magnetic field, was unobservable. Now with SXR and EUV imaging one can study essentially the same structures via these thermal emissions (adding to what one can learn from radio techniques; see White et al. 2011, for further information).

A flare and/or CME disrupts the coronal structure in ways that are not completely clear yet, and in the process of this large-scale dynamics there is powerful particle acceleration. It is impossible in this short review to do justice to the vast number of studies of CMEs both at the Sun and in interplanetary space, and so we focus here on how the flare, the CME and the related particle acceleration fit together observationally, a process sometimes likened to the fable of the blind man and the elephant (e.g., Hudson \& Cliver 2001; Cliver \& Hudson 2002, where much more extensive and amusing reviews of the flare/CME relationship can also be found).

A flare/CME event marks the conversion of stored magnetic energy into various other forms that propagate through the solar atmosphere and into interplanetary space. Traditionally the brightening is associated with the "flare" and the motions with the "CME" (Cliver 1995), but the physics of either phenomenon seems to require both motions and brightenings. Ultimately, all of the energy extracted from the magnetic field appears either as energy associated with the CME, or as enhanced radiative output of the Sun. The dominant term of the CME energy appears to be its kinetic energy, which can be estimated from LASCO images (Vourlidas et al. 2000). The dominant energy product of a flare, of course, is the 


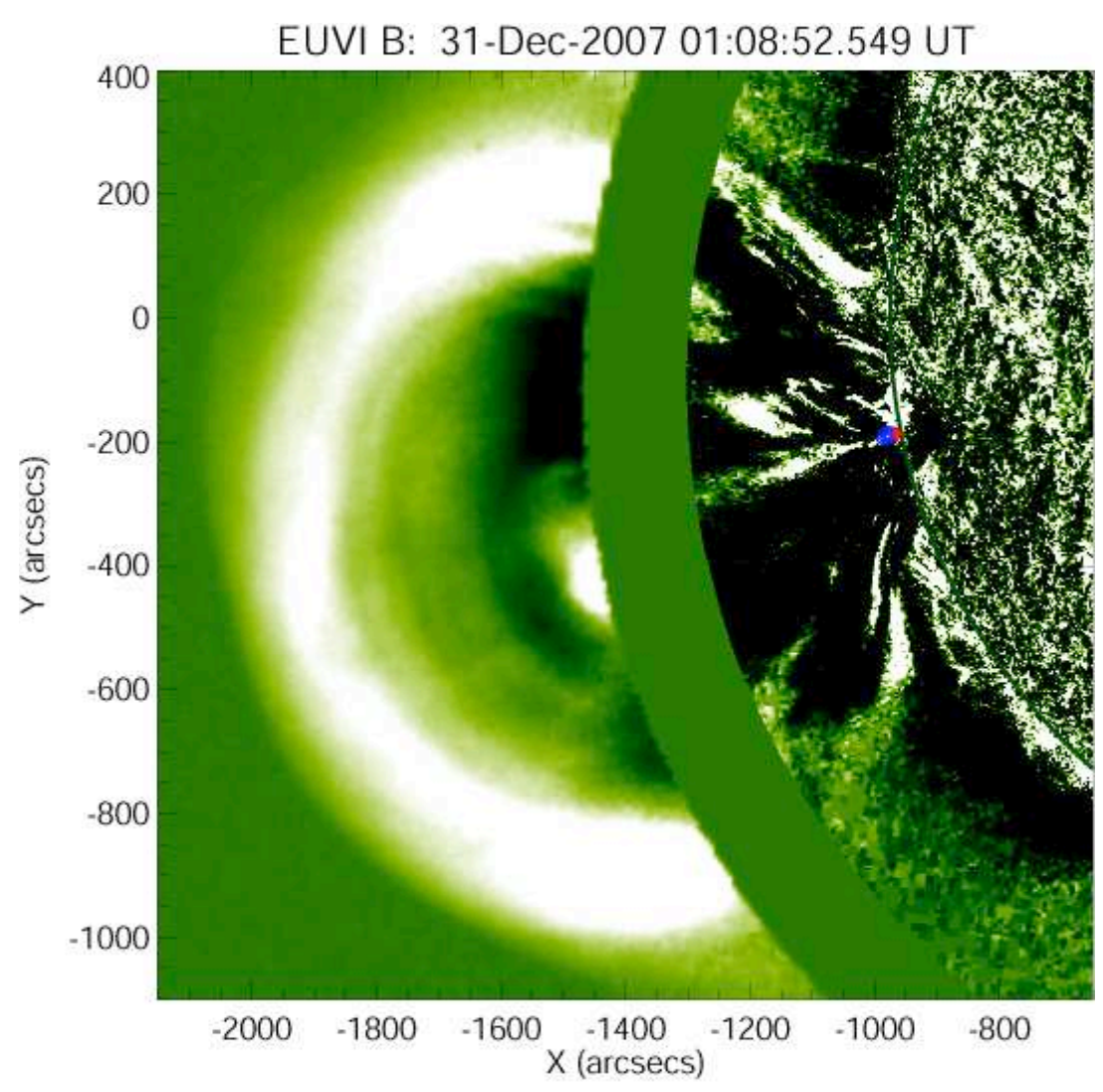

Fig. 5.1 A combined RHESSI/STEREO image of SOL2007-12-31T01:11 (C8.3). It is composed of a difference image of the solar disk and inner corona, which illustrates well the loss of coronal plasma referred to as the "dimming," RHESSI sources (red and blue contours) and the CME bright front, dark following cavity and inner bright core. As is apparent, a CME is a very much more spatially extensive phenomenon than is the flare (image courtesy S. Krucker).

transient excess it makes in the solar luminosity. We discuss this in detail in Section 6.2 . One immediately evident property of the flare/CME combination is the comparative sizes of the flaring region and the associated CME, illustrated quite startlingly in Figure 5.1

\subsection{Flare energy release and CME dynamics}

From reconnection models it is supposed that the CME kinematics and the energy release of the associated flare are closely related. The degree of association between flares and CMEs had always been problematic, largely because of the poor coverage of the low corona provided by coronagraphs. Almost half of the CMEs, for example, originate on the far side of the Sun, for which no low-coronal observations had been available until the advent of STEREO. Nevertheless the X-ray observations of coronal dimmings (Hudson et al. 1996) and EUV observations of compact CME sources (Dere et al. 1997a) had made it clear that there was often a very tight relationship between flares and CMEs. 


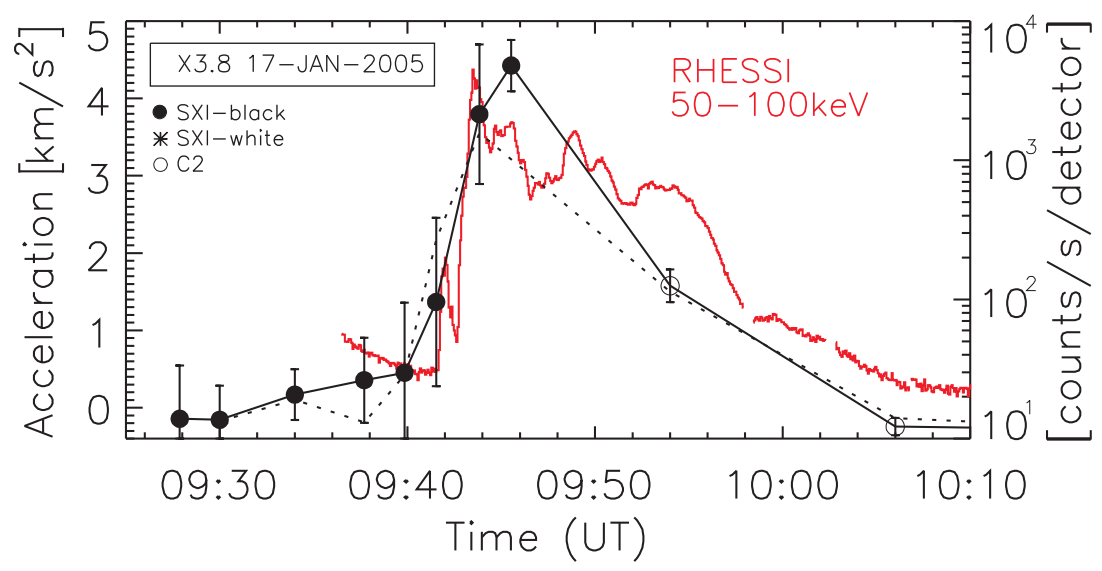

Fig. 5.2 Flare HXR flux (RHESSI 30-100 keV) and CME acceleration profile derived from GOES/SXI SXR images and SOHO/LASCO coronagraph images for SOL2005-01-17T09:52 (X3.8). Note the close synchronization (Temmer et al. 2008).

Further studies revealed a close correlation between the CME acceleration and the derivative of the flare SXR flux, taken as a proxy for the flare energy release (Zhang et al. 2001, 2004; Maričić et al. 2007). For some well-observed CME/flare events, further direct evidence was recently provided showing a very close synchronization between the CME acceleration and the RHESSI HXR flux. For example, in the SOL2005-01-17T09:52 (X3.8) flare/CME event and in SOL2006-07-06T08:36 (M2.5) (Temmer et al. 2008), the use of GOES/SXI and TRACE running difference images showed that the CME impulsive acceleration in the low corona and the flare energy release (deduced from the RHESSI HXR flux) are closely synchronized, and peak simultaneously within \pm 3 min, i.e., within the CME measurement uncertainties (see Figure 5.2). Such correlations provide strong evidence that the CME large-scale acceleration and the flare particle acceleration are intimately connected phenomena, reflecting the rapid extraction of energy from the reconfiguring field both below and above the coronal reconnection region. The effect of the magnetic boundary conditions (i.e., with field being line-tied to the photosphere below the coronal reconnection region, and able to expand relatively freely above) obviously has a substantial impact on the energetically dominant terms in the flare versus the CME.

\subsection{Large-scale waves}

The restructuring of the large-scale coronal magnetic field implicit in a flare and a CME can be considered as a magnetic impulse, and the magnetic field that permeates the surrounding corona and deeper atmosphere guarantees that large-scale waves will ripple away from the site (for a recent review of coronal waves see Vršnak \& Cliver 2008). Because of dispersion, any of the global waves will shock and dissipate their energy in non-thermal effects, such as the acceleration of few-keV electrons in a radio type II burst. The waves may contain large energies, as discussed in Section 6.3. In general an analysis of the structure and timing of these waves may provide key clues to the nature of the energy release by helping to define its geometry. 
The existence of large-scale coronal shock waves has in fact been known since the early interpretation of the "slow drift" or metric type II radio bursts (e.g., Wild et al. 1963). These were then linked to the Moreton waves, which are observed in chromospheric $\mathrm{H} \alpha$ signatures (Athay \& Moreton 1961), by the theory of a weak fast-mode MHD shock (Uchida 1974). Figure 5.3 shows a well-observed example of a Moreton wave, which typically travels across the chromosphere at $1000 \mathrm{~km} \mathrm{~s}^{-1}$. Even before this time "flare ejecta" or "driver gas," now known as an ICME (for Interplanetary CME), had been identified as the cause of the "storm sudden commencement" geomagnetic effect. This is the abrupt onset of a magnetic storm resulting from the compressive interaction of an interplanetary shock wave driven by what we now term an ICME (e.g., Caroubalos 1964) with the magnetosphere Now in addition to this well-known coronal and chromospheric evidence of large-scale waves, we can add the "EIT wave" observations (Moses et al. 1997; Thompson et al. 1998). These EUV perturbations take the form of an expanding wave front most clearly visible in difference images. In a small subset of the EIT waves one can make an identification with the Moreton wave/type II phenomenon, but for the most part they have clearly different properties (Biesecker et al. 2002). Their interpretation in terms of X-ray, EUV or white light dimming (or depletion) (e.g., Hudson et al. 1996; Sterling \& Hudson 1997) is complicated because of the temperature sensitivity of the EIT response. The EIT signature is presumably a mixture of true depletion, simple waves, and large-scale restructurings of the field as required by the CME.

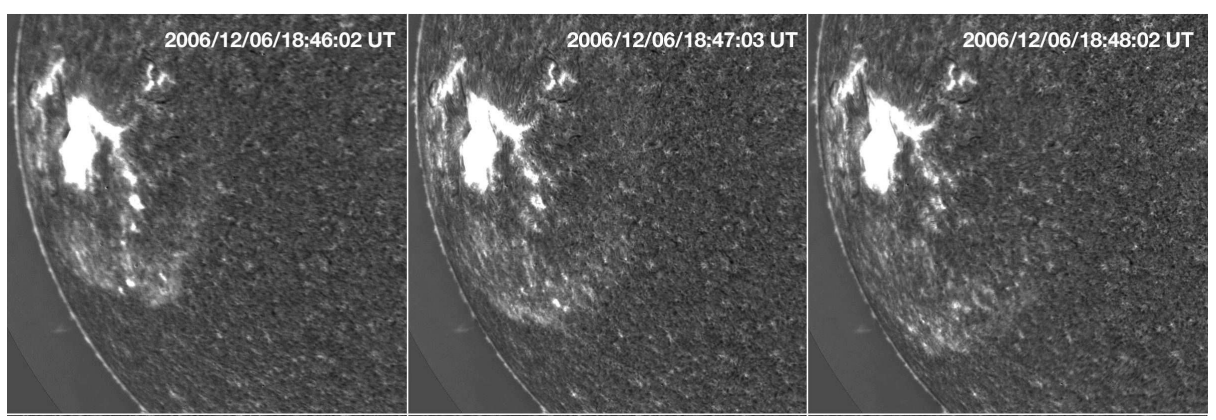

Fig. 5.3 Moreton wave observed in $\mathrm{H} \alpha$ at Sacramento Peak in SOL2006-12-06T18:47 (X6.5). Courtesy K. S. Balasubramaniam.

The similarity of the radio signatures (metric type II for the flare-associated wave, and interplanetary type II for the CME-driven wave) have led to much recent discussion (see Pick et al. 2006, for a recent comprehensive survey) regarding the distinction, if any, between the meter-wave and the interplanetary shock signatures. The radio spectrograms at long and short wavelengths each have complex signatures, and have been traditionally made in disjoint spectral bands. It has thus been common (e.g., Cliver et al. 2004) to speculate that a common mechanism, specifically the CME bow wave, could explain all of the large-scale wave observations. Indeed, the coronagraphic observations show image evidence, in many cases, for CME-driven shocks in the middle corona (Vourlidas et al. 2003; Ontiveros \& Vourlidas 2009). However there is no clear evidence for continuity in the radio signatures, using new observations in the 1-14 MHz range that separates the traditional ground-based and interplanetary observations (Cane \& Erickson 2005). The distinction between a flare origin and a CME origin has also become more difficult to make now that improved data have established tighter relationships between flares and CMEs in both point of 


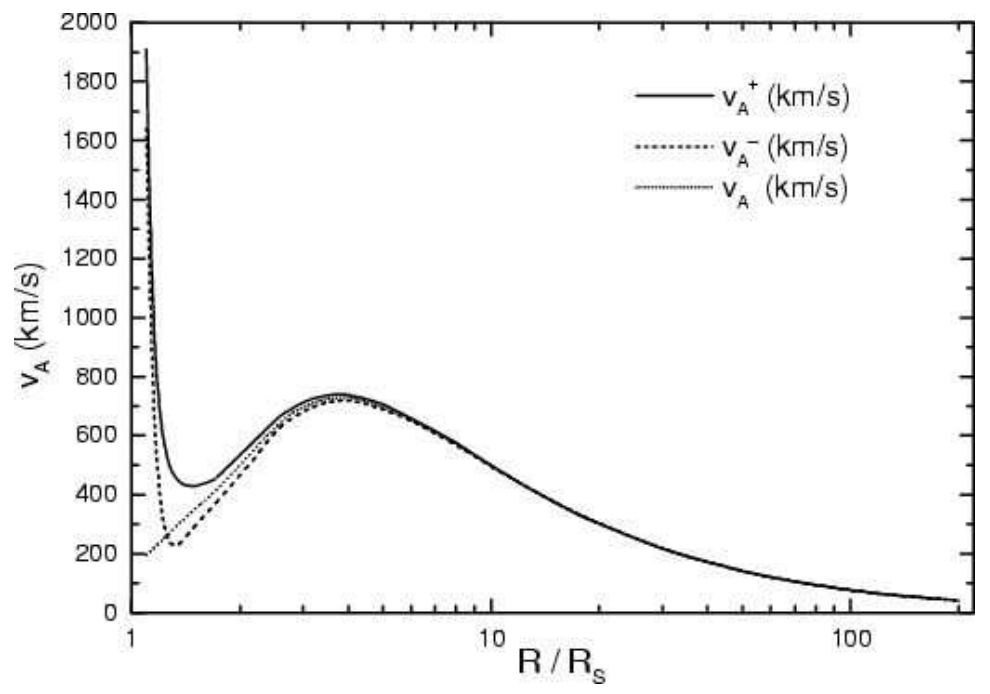

Fig. 5.4 Model variation of Alfvén speed with height in the corona, showing the local minimum above an active region (Mann et al.2003). Here the dotted line shows the quiet Sun, modeled as a simple dipole at Sun center, and the active region as a vertical dipole near the surface. The solid and dashed lines show the cases where these two components have parallel or antiparallel radial contributions.

origin and timing (see Figure 5.2). The coronagraph data show the importance of the flanks of the CME-driven wave, both directly and also indirectly via the excitation of streamers that the flanks intersect. SXR observations may show the earliest signatures of the largescale wave disturbance (Khan \& Aurass 2002; Hudson et al. 2003).

\subsection{Solar Energetic Particles and particle acceleration}

Shock waves can be efficient accelerators of high-energy particles, and there is clear (though indirect) evidence for shock acceleration of SEPs (e.g., Reames 1999). Indeed, the energy ending up in energetic particles can be a substantial fraction of the CME kinetic energy (see Section 6.3). The shock acceleration of SEPs probably takes place at some distance from the event origin (Kahler 1994). This would be consistent with the idea that the shock condition does not develop immediately, presuming that larger Mach numbers correspond to more efficient acceleration. The existence of a minimum in the Alfvén speed in the middle corona (Mann et al.2003) allows the CME-driven disturbance to attain a higher Mach number even as its absolute speed may be constant or even decreasing. Figure 5.4 shows a model view of the coronal Alfvén speed (Mann et al. 2003).

A more directly flare-related acceleration of SEP ions may also occur, and the fact that SEP occurrence is strongly associated with a "soft-hard-harder" spectral evolution (Kiplinger 1995; Grayson et al. 2009) may support this more direct connection. Solar electron events detected in interplanetary space have a strong association with metric-decimetric type III bursts (e.g., Lin 1970). Here the starting frequency of the radio emission, taken to be the plasma frequency or its first harmonic, points to relatively high densities (the lower corona) consistent with a process physically close to the flare site. 


\section{Physical properties of flares}

\subsection{X-ray spectroscopy}

The emission spectra of flares, especially in the SXR range, convey the most direct information obtainable by remote sensing of the flaring coronal plasmas. RHESSI touches on this domain via its capability for measuring the thermal free-free and free-bound continua, as well as to detect the K-shell emission lines of highly ionized Fe around $6.7 \mathrm{keV}$. These spectral features appear commonly in a wide variety of astrophysical sources, such as active galactic nuclei, stellar flares, and supernova remnants. The parameters thus available include the electron temperature $T_{e}$, the emission measure $n_{e} n_{i} V$, and information about elemental abundances given adequate models of the plasma physics and the atomic physics. RHESSI data, for example, can determine the abundance ratio $\mathrm{Fe} / \mathrm{H}$ from the equivalent width of the Fe-line feature at $\sim 6.7 \mathrm{keV}$ (a measure of the ratio of the Fe-line flux to the continuum flux at the Fe-line energy). Note that the Fe feature actually consists of many lines in the K-shell energy range, as described by Phillips (2004). The solar Fe K-shell spectral feature typically does not exhibit the $\sim 6.4 \mathrm{keV}$ emission of Fe in low ionization states. This is commonly observed elsewhere in the Universe, for example, in reflection spectra from accretion disks, but is largely absent in the solar spectra (in favor of the $\sim 6.7 \mathrm{keV}$ emission band due to high-temperature plasmas).

Thermal continua observed by RHESSI: The flare thermal spectrum observed by RHESSI in the $\sim 3-20 \mathrm{keV}$ energy range consists of free-free (bremsstrahlung) and free-bound (recombination) continuum emission. The contributions made by these continua vary with energy and $T_{e}$. In general, thermal free-free radiation is predominant at lower energies and higher temperatures, as was evident in early calculations (Culhane 1969; Gronenschild \& Mewe 1978). Newer continuum calculations are included in the Chianti atomic database and software package (Dere et al. 1997b; Young et al. 2003), but the dependence on energy and temperature is nearly the same as the earlier work. There is now, however, the recognition that the abundances of some elements important for free-bound emission are enhanced in the corona, giving rise to enhanced free-bound emission. For flare temperatures between 10 and $20 \mathrm{MK}$ and with coronal element abundances, the cross-over energy where free-free and free-bound continua fluxes are equal is at the lower end of the range that RHESSI observes, so both free-free and free-bound continua are important (e.g., White et al.2005b). (The twophoton continuum, due to the de-excitation of metastable levels in H-like and He-like ions, is much less important.)

The Fe-line and Fe/Ni-line features observed by RHESSI: As well as the continuous emission, RHESSI observes two line features at $\sim 6.7 \mathrm{keV}$ and $\sim 8 \mathrm{keV}$, known as the Feline and $\mathrm{Fe} / \mathrm{Ni}$-line complexes. They are composed of numerous individual spectral lines emitted mainly by He-like Fe XXV ions and dielectronic satellite lines emitted by mainly Li-like Fe XXIV and lower Fe ions, with a small contribution from highly ionized Ni lines to the $\sim 8 \mathrm{keV}$ feature. In coronal ionization equilibrium, these ions are expected to be abundant at temperatures above $\sim 10 \mathrm{MK}$, and in confirmation of this, the Fe-line feature is evident in RHESSI spectra with $T_{e} \gtrsim 10 \mathrm{MK}$. Both line features are conspicuous for $T_{e} \gtrsim 20 \mathrm{MK}$. The spectral resolution of the RHESSI detectors at these energies is $\sim 1 \mathrm{keV}$ FWHM, which is insufficient for resolving the line structure of the Fe-line and Fe/Ni-line features. Nevertheless, RHESSI has the advantages of covering a much broader energy range than previous high-resolution crystal spectrometers (which have only covered the immediate vicinity of 
the $\sim 6.7 \mathrm{keV}$ lines) and by directly observing the continuum - some crystal spectrometers have had a strong background due to crystal fluorescence, which can obscure the true flare continuum. A valuable diagnostic means for studying the hot component of the solar flare plasma is thus available (Phillips 2004).

Temperature, line equivalent width and abundance analysis has been carried out for SXRs ( $\gtrsim 5 \mathrm{keV}$ ) spectra taken during 27 flares observed by RHESSI, with GOES class between C3 and X8 (Phillips et al. 2006). The measured spectra were fitted with model spectra consisting of a continuum (isothermal free-free plus free-bound emission) and lines at $\sim 6.7 \mathrm{keV}$ to characterize the Fe-line feature and at $\sim 8 \mathrm{keV}$ for the Fe/Ni-line feature. Figure 6.1 shows an example of this during SOL2003-04-26T03:06 (M2.1), at a time when RHESSI was in its single-attenuator state 14 . The temperatures $T_{e}$ in this analysis ranges between $11 \mathrm{MK}$ and $29 \mathrm{MK}$ (Phillips et al. 2006).

The equivalent widths of the Fe-line and $\mathrm{Fe} / \mathrm{Ni}$-line features were also derived. Many flares had were long enough to allow repeated measurements of particularly the Fe-line equivalent width, mostly during the flare decay phase. All the measurements were made with the thin RHESSI attenuator in place. An example of measured equivalent width variations during SOL2002-05-31T00:16 (M2.4) is shown in Figure 6.2 Similar calculations were done for the $\sim 8 \mathrm{keV} \mathrm{Fe} / \mathrm{Ni}$-line feature. Both are based on a coronal $\mathrm{Fe} / \mathrm{H}$ abundance ratio of $1.26 \times 10^{-4}$ (Feldman \& Laming 2000), or 4 times the photospheric value. These are compared with theoretical calculations of the equivalent width vs. temperature. There is a general agreement in the trend for both the $\mathrm{Fe}$-line and $\mathrm{Fe} / \mathrm{Ni}$-line equivalent widths. This indicates that the coronal $\mathrm{Fe}$ abundance is appropriate for this flare, though there is a systematic displacement of the points towards higher temperatures. This may be due to the multithermal nature of the flare plasma, or to the presence of non-thermal effects, or to instrumental effects at high photon count rates. They may also be due to incorrect atomic rates used in the calculation of the He-like Fe XXV ion fractions in ionization equilibrium calculations, since for most of the temperature range shown the fraction of $\mathrm{Fe} X X V$ ions is small $(\lesssim 0.3)$ where the uncertainties are greatest.

The measured $\mathrm{Fe} / \mathrm{H}$ ratios for up to 22 of the 27 flares when in the first RHESSI attenuator state were found to be consistent with a $\mathrm{Fe} / \mathrm{H}$ abundance ratio between 0.8 and 1.0 times the coronal value. No large-scale time variations in the $\mathrm{Fe} / \mathrm{H}$ abundance are apparent, as were derived for $\mathrm{Ca} / \mathrm{H}$ abundance ratios from the BCS instrument on SMM (Sylwester et al. 1984, 1998). Ratios measured in the thickest RHESSI attenuator state were up to nearly a factor of two higher than the theoretical curve, but for these there was generally a poor spectral fit. The best agreement of measured equivalent widths are for spectra taken with RHESSI in its first attenuator state during flare decay stages, for which spectra were apparently more nearly isothermal than near the flare peak and rise stages.

Ratio of Fe-line to Fe/Ni-line in RHESSI spectra: The Fe-line feature at $\sim 6.7 \mathrm{keV}$ in RHESSI spectra is made up of Fe XXV lines and Fe XXIV satellite lines, both emitted as a result of transitions like $1 s-2 p$ or $1 s-2 s$, whereas most $(\sim 85 \%)$ of the Fe/Ni-line feature at $\sim 8 \mathrm{keV}$ is made up of Fe XXV lines and Fe XXIV satellites with $1 s-n p(n \geqslant 3)$ transitions (the remaining $15 \%$ being due to highly ionized Ni lines). The flux ratio of the Fe-line to the Fe/Ni-line features should therefore be sensitive to $T_{e}$ because of the different excitation energies of the transitions, and so $T_{e}$ could be derived independently of the temperature from the continuum emission which might be contaminated with non-thermal emission near

14 RHESSI achieves great dynamic range via two attenuators, which incrementally (in four combinations, but in actual practice only three) cut off the intense low-energy fluxes of major events and also allow the sensitive detection of weak microflares (Lin et al. 2002). 


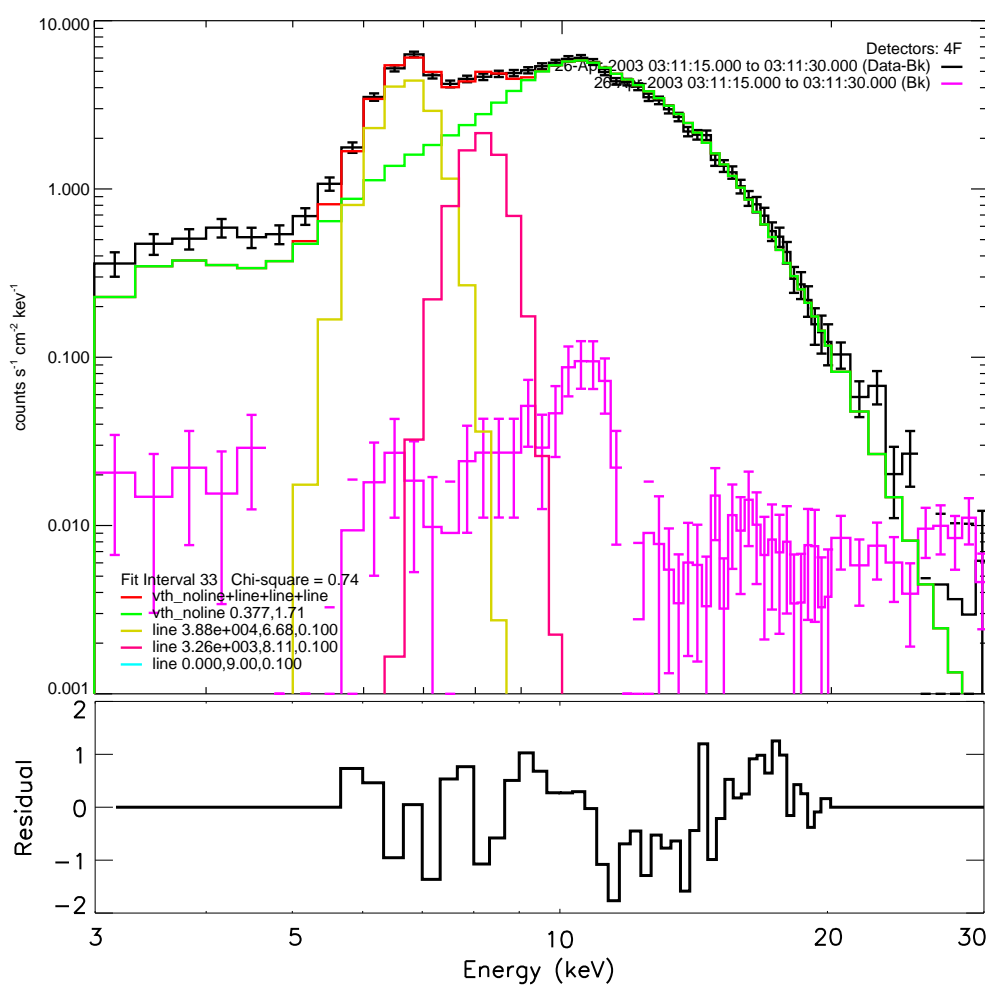

Fig. 6.1 Top: measured and modeled count rate spectra in the energy interval 3-30 keV for RHESSI detector 4 shortly after the peak of SOL2003-04-26T03:06 (M2.1). The measured background-subtracted spectrum in the interval $03: 11: 15-03: 11: 30$ UT is the black histogram with $\pm 1 \sigma$ uncertainties in each energy bin. The background spectrum is the purple histogram with error bars at count rates between $\sim 0.03$ and 0.1 counts $\left(\mathrm{cm}^{2} \mathrm{~s} \mathrm{keV}\right)^{-1}$. The green histogram shows the thermal continuum calculated with the MEKAL atomic code (Meyer 1985) and folded through the spectral response matrix of the RHESSI instrument. The histograms with yellow and magenta lines are two Gaussian line features representing the Fe-line feature $(\sim 6.7 \mathrm{keV})$ and the Fe/Ni-line feature $(\sim 8 \mathrm{keV})$ respectively, while the red histogram represents the total model. The fit range was 5.7 to $20 \mathrm{keV}$, and the reduced $\chi^{2}$ of the fit was 0.74 . Bottom: residuals in the fit range plotted as the number of standard deviations.

the flare impulsive stage. The agreement of the observed and calculated equivalent widths of both line features (Figure 6.2) indicates that for most RHESSI spectra the continuum temperature (which is plotted as the abscissa in Figure 6.2) describes both equivalent widths well, so that the temperature from the ratio of the line features is nearly equal to the continuum temperature.

For SOL2002-07-23T00:35 (X4.8), the continuum spectrum is well-fit by two thermal components throughout the impulsive and decay phases. However, the Chianti predictions (cf. Phillips 2004) of the Fe and Fe/Ni line fluxes and ratio based on the observed temperatures and emission measures of the two thermal components are significantly larger than the measured line values - by, on average, $\sim 55 \%, \sim 20 \%$, and $\sim 34 \%$, respectively, with larger deviations at lower continuum temperatures (Caspi \& Lin 2010). These discrepancies suggest against the interpretation of non-thermal excitation for the lines (as that would produce fluxes in excess of the predictions); while abundance variations could explain the deviations 


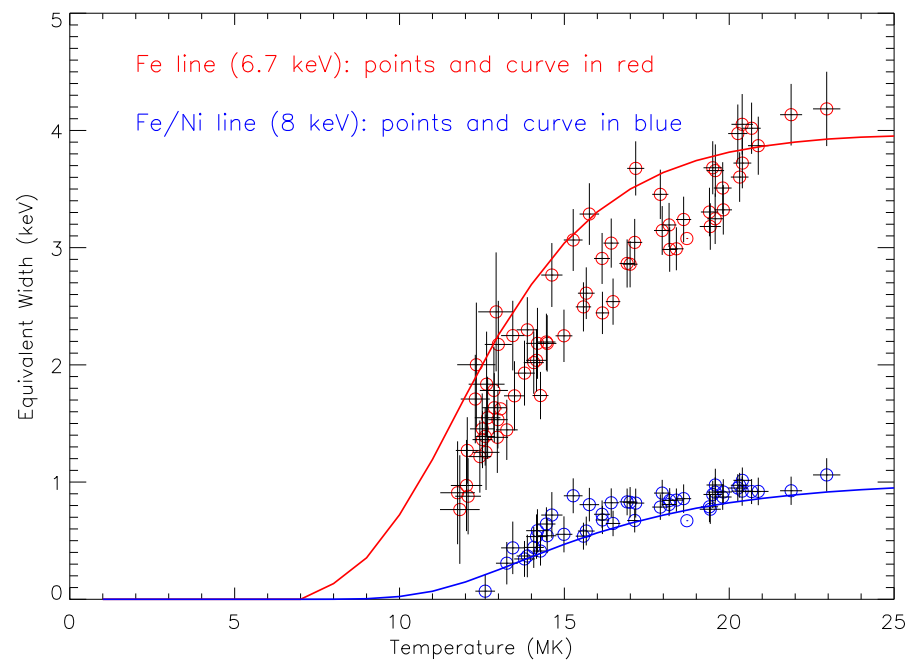

Fig. 6.2 Measured and predicted equivalent widths (see text) as a function of $T_{e}$ for SOL2002-05-31T00:16 (M2.4). The red upper points are for the Fe-line feature with $\pm 1 \sigma$ error bars obtained from spectral fits similar to the one shown in Figure 6.1 The blue lower points show the Fe/Ni-line feature. The corresponding solid curves show the theoretical dependence, updated from Phillips (2004). Atomic data used is from the Chianti database (v. 5.1) (Dere et al. 1997b; Young et al. 2003) (from Phillips et al. (2006).

of the line fluxes, the variation in the ratio is more likely explained by ionization-fraction uncertainties as suggested by Phillips et al. (2006).

Non-isothermal effects in RHESSI spectra: As indicated earlier, the analysis of Fe-line emission in RHESSI spectra to derive the flare Fe/H abundance ratio (Phillips et al. 2006) was based on the assumption that the emitting plasma was isothermal. This conflicts with the appearance of flares with multiple loops, in which each loop may have a different $\mathrm{T}_{e}$. In spite of this the isothermal approximation appears to apply to many flares, even including long-duration flares observed spectroscopically from Yohkoh in S XV and Ca XIX lines (Phillips et al. 2005). Such flares have obvious multiple-loop structures. The success of isothermal fits also applies to RHESSI spectra at low energies, for which good fits to spectra obtained even with the RHESSI attenuators in place are generally achieved. For the rise phase of many flares, however, fits to RHESSI spectra are not so satisfactory, even for spectra which appear not to have any non-thermal component in the low-energy continuum. Inspection of RHESSI images and the higher-resolution images from TRACE and $\mathrm{SOHO/EIT} \mathrm{instrument} \mathrm{at} \mathrm{the} \mathrm{developing} \mathrm{stages} \mathrm{of} \mathrm{flares} \mathrm{shows} \mathrm{that} \mathrm{many} \mathrm{individual} \mathrm{loop}$ structures contribute to the total flare emission, perhaps with different temperatures.

Analysis of the differential mission measure (DEM) is therefore required, or at least RHESSI imaging spectroscopy, for these initial flare stages Although the parameters of the DEM are difficult to determine from RHESSI spectra alone, some progress can be made using simple forms for the temperature structure of the developing flare plasma, such as DEM $=T_{e}^{-\alpha}$ or $\exp \left(-T_{e} / T_{0}\right)$ where $\alpha$ or $T_{0}$ characterize the emitting plasma at any particular time. A more physically based description would involve a set of nearly isothermal components reflecting the multiple loops each in its own cooling equilibrium, so that the DEM 
parameters $\alpha$ or $T_{0}$ would characterize the distribution of the components needed. However, Caspi \& Lin (2010) showed that for at least one flare (SOL2002-07-23T00:35), the spectrum is well represented by two isothermal components, and a DEM analysis is consistent with a bimodal DEM. Cursory analysis of other X-class flares reveals a similar bimodal structure. A simple exponential or power-law DEM is therefore not necessarily a reasonable approximation.

Non-thermal excitation of continuum and line emission in RHESSI spectra: It has been commonly assumed that the non-thermal electrons accelerated during the flare impulsive stage produce bremsstrahlung (free-free radiation) as they interact with the chromospheric or coronal material. The free-free emission is basically due to hydrogen, with small contributions from $\mathrm{He}$ atoms or ions, reflecting the solar composition. Free-bound radiation from the non-thermal electron continuum has until recently been neglected, on the grounds that high-energy electrons are less likely to recombine on ions than they are to emit free-free radiation. This has now been questioned (Brown \& Mallik 2008, 2009), who argue that for Fe particularly the free-bound radiation may be very important for hot flare sources such as limb flares in which the footpoints are occulted .

This little-studied effect could considerably alter the emission for such flares, and should be taken into account where it is detectable. This mechanism is fundamentally different from free-free emission in that the emitted X-ray energy maps one-to-one with the energy of the parent electron, rather than as an integral over the entire distribution. This means that its spectral features in principle can be interpreted much more directly.

As well as non-thermal effects on the flare continuum, non-thermal electrons may give rise to excitation of lines such as those making up the Fe-line feature at $\sim 6.7 \mathrm{keV}$. Excitation could occur by the ionization of K-shell electrons in near-neutral $\mathrm{Fe}$, with re-arrangement of the Fe atoms and emission of Auger electrons (67\%) or photons (33\%), resulting in the $\mathrm{K} \alpha$ or $\mathrm{K} \beta$ lines (inner-shell transitions $1 s-2 p$ and $1 s-3 p$ respectively). An energy of at least $7.1 \mathrm{keV}$ is required for the removal of the K-shell electron in each case. Observations of these lines (at $6.4 \mathrm{keV}$ for $\mathrm{K} \alpha$ and $7.1 \mathrm{keV}$ for $\mathrm{K} \beta$ ) could provide a diagnostic for a non-thermal electron distribution that has sharp cut-off energy $E_{0}$, since the lines would not be observed for $E_{0}>7.1 \mathrm{keV}$ but would if $E_{0}<7.1 \mathrm{keV}$ (Phillips \& Neupert 1973). In practice a sharp cut-off would be quickly smoothed out by interaction of the lower-energy electrons in the distribution with ambient plasma. Most of the observed $\mathrm{Fe} \mathrm{K} \alpha$ line emission is due to fluorescence of neutral $\mathrm{Fe}$ in the photosphere for disk flares (Bai 1979; Parmar et al. 1984) and not to K-shell ionization by electrons. However, an intriguingly marginal case was observed with the BCS instrument on $S M M$ in which excitation by non-thermal electrons might have been significant (Emslie et al. 1986).

Summary: RHESSI was intended primarily as a probe of the non-thermal emission spectra of flares at high energies, but observation of low-energy $(\sim 3-20 \mathrm{keV})$ flare spectra has yielded important information. Results include the derivation of electron temperature evolution during the peak and decay stages of flares from the thermal continuum based on an isothermal assumption, though simple approximations to the temperature distribution have also been used. The continuum is theoretically due to free-free and free-bound radiation in comparable amounts in the RHESSI energy range. The two line features, the Fe-line (at $\sim 6.7 \mathrm{keV})$ and $\mathrm{Fe} / \mathrm{Ni}$-line $(\sim 8 \mathrm{keV})$ features, enable the abundance of Fe relative to $\mathrm{H}$ to be determined from their fluxes relative to nearby continuum emission. Analyses of spectra during the peak and decay phases of flares suggest a coronal value of $\mathrm{Fe} / \mathrm{H}$, i.e., one that is larger than the photospheric value by a factor $2-4$. This is confirmed by measurements from 
the SOXS instrument on the GSAT-2 spacecraft (Jain et al. 2006). Additional temperature information is offered by the flux ratio of the two line features: generally the temperatures derived are similar to those obtained from the energy dependence of the thermal continuum. Nonthermal effects are currently being investigated particularly for the Fe-line and $\mathrm{Fe} / \mathrm{Ni}$-line features, and could be a sensitive probe of the low-energy cut-off energy in the non-thermal electron distribution if one exists.

\subsection{Flare energetics}

In the matter of constructing the overall energetics of a flare and its associated mass ejection, the requirement for multi-wavelength observations is clear. Direct measurements of the total radiative output are available for only a few flares, notably SOL2003-10-28T11:10 (X17.2) from the Total Irradiance Monitor on the SORCE spacecraft (Woods et al. 2004). While such measurements are the most accurate for estimating the total energy released in an event, information about the nature of the energy release process itself can be acquired only through analysis of the partition of energy amongst the various components such as energetic particles and thermal plasma that are present as the flare proceeds.

The first attempts to estimate the total irradiance excess from a flare were made with the Active Cavity Radiometer Irradiance Monitor (ACRIM) on board the Solar Maximum Mission. These observations, unfortunately, yielded only upper limits (Hudson \& Willson 1983). The TIM instrument currently flying on SORCE has now made definite observations (Woods et al. 2004, 2006), and these have proven to be a key factor in our new ability to characterize the partition of energy since they provide a direct measure of the total flare radiation. These results may improve with time if filtering can be developed to reduce the TIM background fluctuations. However the best way to measure this important parameter sensitively could be to have imaging bolometric measurements, which would avoid the large background fluctuations due to p-modes and convective motions in the rest of the photosphere.

Considerable constraints on the energy release processes follow from a consideration of the partition of the released energy between accelerated particles, radiation, heated plasma, and ejected solar material. But any exercise of this sort must be done rather carefully in order not to "double count" energy terms that are directly related to each other, e.g., energy in accelerated electrons that is used to produce thermal plasma (Emslie et al. 2005) or radiation. This requires distinguishing amongst "primary" components of energy (e.g., the magnetic field), "intermediate" components (e.g., accelerated particles and thermal plasma), and "final" components (e.g., kinetic energy of ejecta, radiant energy in various wavebands), and recognizing the overlap of these components.

In the RHESSI era, the partitioning of energy in two well-observed solar flare/CME events was carried out using data from a variety of missions including RHESSI, ACE, SOHO and GOES. This study yielded the result that "flare radiant energy and CME mechanical energy are the same order of magnitude." The SXR flare (from $1.5 \mathrm{keV}$ ) appears to contain substantially less than about $10 \%$ of the total radiant energy (Emslie et al. 2005). The impulsive-phase radiation appears to dominate the flare luminosity. Both the SEPs and the impulsive phase acceleration contain a substantial fraction of the total energy (Emslie et al. 2005), as described below. 
Table 6.1 Flare and CME Energy Budgets*

\begin{tabular}{|c|c|c|}
\hline & 21 April 2002 & 23 July 2002 \\
\hline \multicolumn{3}{|l|}{ Primary Energy } \\
\hline Magnetic & $32.3 \pm 0.3$ & $32.3 \pm 0.3$ \\
\hline \multicolumn{3}{|l|}{ Flare } \\
\hline \multicolumn{3}{|l|}{ Intermediate Energies } \\
\hline Electrons $\left(>E_{\min }\right)$ & $31.3 \pm 0.5$ & $31.3 \pm 0.5$ \\
\hline Ions $\left(>1 \mathrm{MeV}\right.$ nucleon $\left.^{-1}\right)$ & $<31.6$ & $31.9 \pm 0.5$ \\
\hline Thermal Plasma $(T>5 \mathrm{MK})$ & $31.1_{-10}^{+0.4}$ & $30.4_{-10}^{+0.4}$ \\
\hline \multicolumn{3}{|l|}{ Radiant Energy } \\
\hline From GOES plasma & $31.3 \pm 0.3$ & $31.0 \pm 0.3$ \\
\hline Assuming $L_{\text {total }} / L_{X}=100$ & $32.2 \pm 0.3$ & $32.2 \pm 0.3$ \\
\hline \multicolumn{3}{|l|}{ CME } \\
\hline Kinetic & $32.3 \pm 0.3$ & $32.3 \pm 0.3$ \\
\hline Gravitational Potential & $30.7 \pm 0.3$ & $31.1 \pm 0.3$ \\
\hline Energetic Particles at $1 \mathrm{AU}$ & $31.5 \pm 0.6$ & $<30$ \\
\hline
\end{tabular}

* Tabulated values are $\log _{10}$ of the energies in erg of the different components given in table 1 of Emslie et al. (2005).

\subsection{Energetics of two large RHESSI flares}

A number of previous studies have examined the energy budget of a limited number of energy components in certain flares. The radiative energy budget of SOL1973-02-05 was evaluated by Canfield et al. (1980), but in the absence of HXRs or $\gamma$-ray observations for this event the role of energetic particles in the event could not be assessed. The X-ray and $\gamma$-ray observations of several flares, including the major $\gamma$-ray flare SOL1972-08-04, were used to show that the kinetic energy of the accelerated electrons constituted a surprisingly large fraction of the total flare energy, perhaps as high as 10 to $50 \%$ of the $\sim 10^{32} \mathrm{erg}$ released during the flare (Lin \& Hudson 1976). Two flares within the same active region on $1980 \mathrm{Au}-$ gust 31 provided the energy content in thermal plasma, non-thermal electrons, and hydrodynamic mass motions of non-ejected material (Strong et al. 1984), while the energy content in radiative, thermal, non-thermal electron, and non-CME associated plasma ejected was calculated for SOL2002-02-26T10:27 (C9.6) (Saint-Hilaire \& Benz 2002). RHESSI X-ray observations were used determine the energy in accelerated electrons and in the hot plasma for nine medium-sized flares (GOES class C6 to M8), with the conclusion that despite the large uncertainties, the energies in these two components were of the same magnitude in each case (Saint-Hilaire \& Benz 2005).

The energetics of two X-class flares (SOL2002-04-21T01:51 and SOL2002-07-23T00:35) have been analyzed in a very comprehensive study made possible by overlapping observations at a variety of wavelengths (Emslie et al. 2004, 2005). SOL2002-04-21T01:51 was a long-lived SXR event which occurred near the west limb; SOL2002-07-23T00:35 was much more impulsive, a strong emitter of HXRs and $\gamma$-rays (see Lin et al. 2003), and was located near the east limb at S13E72. Observations were used from instruments on the ACE, SOHO, and RHESSI to provide quantitative estimates of the energy contents of (1) the coronal mass ejection, (2) the thermal plasma at the Sun, (3) the accelerated electrons producing hard $\mathrm{X}$-rays, (4) the accelerated ions producing gamma rays, and (5) the solar energetic particles accelerated by the outward eruptive disturbance/CME. The detailed energy budget for these two events, including the CME kinetic and potential energies and the energy in the SEPs at $1 \mathrm{AU}$, is reproduced in Table 6.1. 
Magnetic energy: The total magnetic energy available for conversion into other forms (flare and $\mathrm{CME}$ ) can be estimated in principle from the extrapolation of the field observed at the photosphere. There are many uncertainties with such a procedure. The flares providing the information in Table 6.1 were each at the limb and therefore not amenable to the extrapolation in any case. Thus we view the uncertainty of 0.3 dex quoted in the table as highly optimistic.

Energy in the CME: To derive the energy in the CME one must first derive a coronal density distribution from the excess brightness (due to Thomson scattering of photospheric light) in coronagraphic white-light images (e.g., from the LASCO C2 and C3 coronagraphs on $\mathrm{SOHO}$; Brueckner et al. 1995). These observations permit an estimate of the mass distribution of the CME (Poland et al. 1981; Vourlidas et al. 2000, 2002). Then, the flow patterns of the plasma during the ejection must be determined from the projected images as a function of time to derive the velocity field of the CME material. Finally these properties can be used to find the potential $\left(U_{\Phi}\right)$ energy, kinetic $\left(U_{\mathrm{K}}\right)$ energy, and enthalpy associated with the CME. For SOL2002-04-21T01:51 and SOL2002-07-23T00:35, the potential energies obtained were $U_{\Phi}=10^{30.7}$ and $10^{31.1} \mathrm{erg}$, respectively, and kinetic energies $U_{\mathrm{K}}=10^{32.3}$ and $10^{32.0} \mathrm{erg}$. These are unusual CME events. Their large kinetic energies place both of them in the top $1 \%$ of all observed CMEs for the period 1996-2000 (Vourlidas et al. 2002). In both cases the gravitational potential energy is $\lesssim 10 \%$ of the total energy contained in the CME. Note that the magnetic energy of a CME, thought to be its dominant term, is almost impossible to assess observationally.

Thermal energy: The thermal energy of the heated plasma is obtained from the temperature $T_{0}(\mathrm{~K})$ and emission measure $E M=\int_{V} n_{\rho}^{2} d V\left(\mathrm{~cm}^{-3}\right)$ for the thermal portion of the overall spectral fit to the HXR data (see, e.g., Holman et al. 2003). Here $n_{e}$ is the electron density $\left(\mathrm{cm}^{-3}\right)$ and $V$ is the emitting volume $\left(\mathrm{cm}^{3}\right)$. Account must be taken of the filling factor $f$ equal to the ratio of the emitting volume to the apparent volume $\left(V_{\text {ap }}\right)$ as determined with an imaging instrument having limited spatial resolution. The thermal energy content of the plasma is then given by

$$
\begin{aligned}
U_{\text {th }} & =3 n_{e} k T_{0} f V_{\text {ap }} \simeq 3 k T_{0} \sqrt{E M \times f V_{\text {ap }}} \\
& =4.14 \times 10^{-16} T_{0} \sqrt{E M f V_{\text {ap }}} \mathrm{erg},
\end{aligned}
$$

where $k$ is the Boltzmann constant and $V_{\text {ap }}$ the source volume, estimated from the area information contained in the RHESSI observations assuming $V_{\text {ap }}=A^{3 / 2}$. For the common assumption of a filling factor of unity $(f=1)$, this is an upper limit for the instantaneous thermal energy. It also can be taken as a lower limit to the total thermal energy since it does not account for the cooling of the plasma prior to a given time, nor for any heating at later times. Each of these contributions could add perhaps a factor of two to the total thermal energy. Application of equation (6.1) to SOL2002-04-21T01:51 and SOL2002-0723T00:35 yielded values of $U_{\text {th }}=10^{31.3} \mathrm{erg}$ and $10^{31.1} \mathrm{erg}$, respectively. An estimate of the total radiated energy can be rather straightforwardly obtained from the GOES SXR data, simply by integrating the product of the emission measure and the optically thin radiative loss function (Cox \& Tucker 1969) (for coronal abundances) over the duration of the flare (Emslie et al. 2005). This exercise gives values of $U_{R} \sim 10^{31.3} \mathrm{erg}$ for the SOL2002-0421T01:51 flare and 10 31.0 erg for SOL2002-07-23T00:35. Note that no knowledge of the source volume, density, or filling factor is required to make this calculation; hence the good 
agreement between these values and those obtained immediately above suggests that the simplifying assumption of unity filling factor is not unreasonable. In particular, the volume filling factor for the soft-X-ray-emitting plasma cannot be too small $(<0.01)$, otherwise the plasma energy calculated using the RHESSI source areas would be significantly below the estimate obtained from the GOES data.

A separate physical argument also suggests that the filling factor cannot be too small. At the time of the peak temperature, the energy density in the thermal plasma, assuming $f=1$, already requires coronal field strengths exceeding $\sim 350 \mathrm{G}$ to contain the plasma; this number increases as $f^{-1 / 2}$ so the filling factor must be no smaller than $\sim 0.01$ unless the coronal field can significantly exceed $\sim 1000 \mathrm{G}$. Caspi (2010) found that this same argument holds for essentially all X-class flares, as they all require coronal field strengths exceeding $\sim 220-460 \mathrm{G}$. This again suggests that a filling factor in the range 0.1-1 is a reasonable assumption for super-hot, X-class flares.

Accelerated Electrons: The energy in accelerated electrons can be determined from applying a thick-target model see (see Holman et al. 2011) to the measured HXR spectrum in order to obtain the injected spectrum $F_{0}\left(E_{0}\right)$ (electrons $\mathrm{s}^{-1} \mathrm{keV}^{-1}$ ) and calculating the corresponding injected power from

$$
U_{\mathrm{e}}=A_{i} \int_{E_{\min }}^{\infty} E_{0} F_{0}\left(E_{0}\right) d E_{0},
$$

where $E_{\min }$ is the lowest particle energy in the non-thermal component of the electron distribution and $A_{i}$ is the injection area (which, however, cancels in further manipulation). The accumulated energy in non-thermal electrons is then obtained by integrating the injected electron power over time. The thermal spectrum is typically dominant at low energies, so the largest value of $E_{\min }$ consistent with an acceptable fit to the spatially integrated spectral data, is chosen; the energies obtained are necessarily lower limits. The values of $U_{e}$ thus determined were $U_{\mathrm{e}}=10^{31.5} \mathrm{erg}$ for SOL2002-07-23T00:35 and $10^{31.3} \mathrm{erg}$ for SOL200204-21T01:51. These results are higher than the corresponding values of $10^{31.3} \mathrm{erg}$ and $10^{31.1}$ erg for the energy contained in the thermal plasma $U_{\mathrm{th}}$. This result is reinforced by the wide lower error bar on $U_{\text {th }}$ caused by the uncertain filling factor $f$ and the fact that $U_{e}$ may be an underestimate. This suggests that much of the electron power is radiated in other wavelengths, such as optical and EUV (see below).

Accelerated Ions: As explained in Vilmer et al. (2011), accelerated ions are also energetically important in energetic solar flares with significant emission above $\sim 300 \mathrm{keV}$ (Ramaty \& Mandzhavidze 2000). The primary ions undergo nuclear collisions and thereby produce $\gamma$-ray lines and continua of various kinds, for example, by direct de-excitation following inelastic scattering to produce lines mainly in the $\sim 1-10 \mathrm{MeV}$ range (e.g., Ramaty et al. 1979). These are broad enough for RHESSI to resolve. There is also a highly-broadened set of lines (a "pseudocontinuum" because of overlaps) resulting from $\alpha$-particles and higher-Z ions striking ambient nuclei. The flux in the highly-broadened component is typically $>3$ times that in the moderately-broadened component. The threshold energies for producing all of these nuclear lines are $\gtrsim 2.5 \mathrm{MeV}$, and so the spectrum below that energy is unknown observationally. Assuming a flat spectrum below $2.5 \mathrm{MeV}$, a lower limit of $(1.0-4.0) \times 10^{30}$ erg of energy and an upper limit of $(1.2-120) \times 10^{32} \mathrm{erg}$ (assuming a power-law spectrum down to $0.1 \mathrm{MeV}$ ) was found for protons in SOL2002-07-23T00:35 (X4.8) (Lin et al. 2003). Protons and heavier ions together range from $U_{\mathrm{i}} \simeq(6-24) \times 10^{30} \mathrm{erg}$ to $(7-700) \times 10^{32} \mathrm{erg}$. For SOL2002-04-21T01:51 (X1.5), for which no significant $\gamma$-ray line emission was produced, 


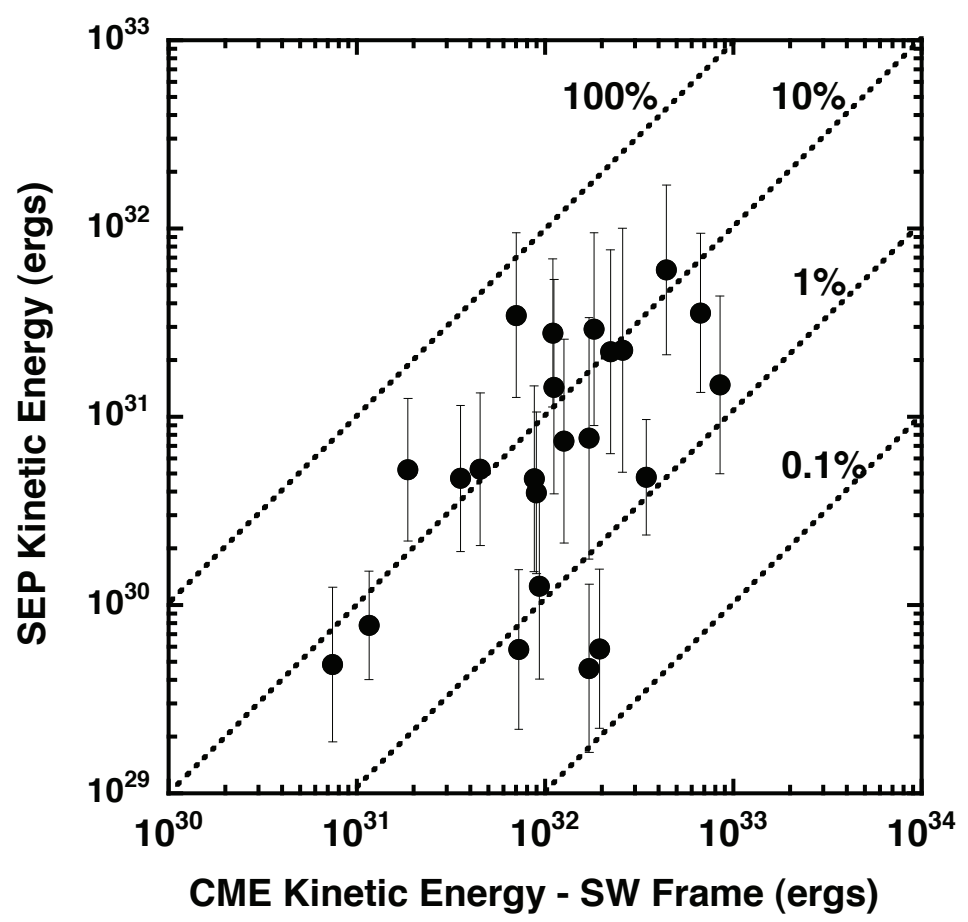

Fig. 6.3 Comparison of SEP energy with CME kinetic energy for a sample of events, adapted from Mewaldt et al. (2008). There is no suggestion of a correlation, but these estimates do imply that the SEPs contain a large fraction of the total CME kinetic energy.

the upper limit is in the range $U_{\mathrm{i}} \simeq 4.0 \times 10^{30} \mathrm{erg}$ to $1.2 \times 10^{34} \mathrm{erg}$, depending on the spectral model used.

Solar Energetic Particles: SEPs accelerated at the flare site and/or at shocks driven by the CME represent another significant contribution to the global energy budget. SOL200207-23T00:35 (X4.8) occurred near the East limb of the Sun (S13E72) and, as is typical for east-limb events, was apparently not magnetically well-connected to Earth. As a result, near-Earth spacecraft such as $A C E$ and GOES did not observe significant SEP fluxes that could be traced to this event. On the other hand, SOL2002-04-21T01:51 at S14W84 was relatively well connected to Earth, and indeed, a strong interplanetary shock (Mach number $\mathrm{M}_{A}=3.7$ ) was observed some two days later, at $\sim 04: 15$ UT on April 23. That the intensities of the SEPs coincided well with the time of shock arrival indicated that acceleration was taking place locally at the shocks. Integrating the energy spectra over energy and solid angle covered by the shock (typically $\sim \pi$ sr) gives the total particle energy incident at $1 \mathrm{AU}$ as $\left.U_{\mathrm{p}} \approx 2.8 \times 10^{31} \mathrm{erg}\right)$, which is a significant fraction $(\sim 15 \%)$ of the CME kinetic energy $\left(\sim 1.8 \times 10^{32} \mathrm{erg}\right)$. This implies that shock acceleration must be relatively efficient. Indeed, a broader survey of SEP events shows that this result holds commonly, though not invariably (Mewaldt et al. 2008). Figure 6.3 illustrates this.

Future improvements: The measurements in Table 6.1 all have sizeable error bars. How can these measurements be refined? We tackle this line-by-line in the table. 
(i) Estimating the primary magnetic energy reliably is a difficult problem, but its solution will certainly involve measurements of the vector magnetic field at the "top" of the chromosphere, with spatial resolution adequate to capture the field close to the magnetic polarity inversion line - scales below one arcsecond. This must be accompanied by fast and robust mathematical methods for the non-linear force-free field extrapolations and/or by direct measurements of the vector field in the corona, e.g., by CoMP or similar instruments.

(ii) Table 6.1 identifies "intermediate energies," reservoirs between the fundamental magnetic field and the true losses (radiation and ejecta). These table entries are themselves model-dependent, for example, in the cooling time scale of the thermal plasma. Detailed EUV-SXR imaging spectroscopy of flare arcades can sharpen these estimates substantially. The current uncertainties on the non-thermal electron energy budget derived from the collisional thick-target model (which is the model requiring the smallest amount of non-thermal particle energy) lie wholly in a lack of knowledge of the "low energy cutoff" of the electron spectrum, if there is one. One theoretical possibility for deducing this information, is to find edges in the X-ray spectrum produced by free-bound radiation (Brown \& Mallik 2008; Brown et al. 2010). No hard X-ray edge structure has yet been reported. The ion (or proton) component is also very poorly constrained, and improving this will require very much better $\gamma$-ray spectroscopy, with much improved signal-to-noise ratio. We also have no knowledge at all of lower energy protons (of a few tens or hundreds of $\mathrm{keV}$ ), though diagnostics such as Doppler-shifted Lyman- $\alpha$ (emitted by charge-exchanging low-energy protons (Orrall \& Zirker 1976) and the (disputed) observations of $\mathrm{H} \alpha$ impact polarization (Vogt \& Hénoux 1999) might help us in this direction.

(iii) Substantial improvement in CME energetics is now being obtained from the STEREO observations. The major uncertainty now, as before, is the lack of knowledge of the magnetic field and its dynamics. Table 6.1 does not even list this item though it is the dominant one.

(iv) The sparse sampling of SEP fluxes, and the difficulty of bookkeeping their distribution in the heliosphere, are some of the issues. Data from nearer the Sun will help, as will improvements in our understanding of their sources. The observation of energetic neutral atoms via charge exchange (Mewaldt et al. 2009) and neutrons, again from inner-heliospheric vantage points if possible, will be a major step forward. Because of the sampling issues, though, this will likely remain inthe domain of statistics rather than precise measurement.

\section{Summary: models and observations}

\subsection{Flare model constraints}

Where within this mass of data can we identify the decisive observations that will choose one model over another? It is a daunting task to find "a flare model" that is capable of explaining all observed phenomena in all events. As we have seen a flare can be geometrically complicated and have many associated temporal components, and its effects appear across a large dynamic range of physical parameters. The extend from the solar interior to beyond one AU. Furthermore, not all flares exhibit the same behavior and many deviate sharply from the "standard" two-ribbon eruptive flare scenario that has framed much of our thinking for decades. For example, some flares do not have associated eruptions, and many have multiple ribbons instead of just two. Some flares continue accelerating electrons well into their X-ray decay phase, some have associated $\gamma$ rays but many - perhaps most - do not.

Despite having orders of magnitude more data than was available at the time that the "standard" scenario was first formulated, our knowledge is still incomplete, and this incom- 
pleteness forces us to invoke cartoons (see http://solarmuri.ssl.berkeley.edu/ hhudson/cartoons/ for a compilation) to relate one observable to another, and to suggest cause and effect. Analytical or computational models with some degree of refinement tend to deal separately with different aspects of the flare; e.g., the magnetic configuration and how it evolves, electron acceleration, expansion of the chromosphere, production of radiation of one type or another. These various parts are then stitched together into cartoons - also loosely called models but without the details of any individual aspect yet being fully worked out.

So, what are the "top-level" components to a flare model? Firstly, we must understand how a particular coronal magnetic configuration can become loaded with or emerge with sufficient stored magnetic energy. We must understand how, after a period of stability, the configuration becomes unstable in such a way as to produce a dramatic energy release. The conversion of stored energy into the various forms which we infer observationally is a perhaps the - central aspect of flare models, which still defies detailed explanation. A fourth model element - undoubtedly also related to the initial magnetic configuration - must address the relationship between the localized radiation burst that is the flare, and the coronal mass ejection. Each of these top-level model components has many sub-components. For example, considering one element of the energy conversion problem, even after 150 years we do not know the origin of flare optical emission. Several alternative theoretical scenarios exist for this part alone, and likewise for the tens of other observed and inferred flare phenomena. Where within this mass of models can we find the decisive predictions?

Let us confine further discussion to the model elements identified as top-level above, and try and identify some ways in which observations support or refute the various options. This is necessarily a very abbreviated discussion as a full comparison between the multifarious models and the observations would require many more pages.

Energy storage: The storage of energy presents an interesting problem. If magnetic reconnection, releasing stored energy, can readily happen in a flare, or indeed in coronal heating, why does it not happen right away? This may come down to the configuration of the magnetic field. Observationally, some progress has been made in understanding energy storage using field extrapolations from observed photospheric vector magnetic fields but these remain problematic, not least because the photosphere is not force-free, in contradiction to the basic mathematical assumptions of the extrapolations. The suggestion from the existing extrapolations, at least of newer active regions, is that free energy storage occurs low down in the atmosphere, close to the polarity inversion line (Régnier \& Priest 2007; Schrijver et al. 2008). Unfortunately, present observations are barely able to resolve the photospheric vector magnetic field, let alone the chromospheric field, at the arcsecond scales required, and the reconstruction techniques are temperamental, so these conclusions should be treated with caution. Nonetheless, many flares also start with their footpoints close to a magnetic neutral line. This often involves the activation of low-lying active region filaments overlying strong sheared polarity inversion lines. This suggests that the properties of what is known as the "core field" in filament models - the strong, twisted or sheared field supporting filament material - are also core to understanding the early phase of flares, and models capable of producing such configurations, whether by shearing or by emergence of structures with concentrated twist, are very relevant. To that end, we will profit from paying close attention to active region filament magnetic field observations, such as those of Wiehr \& Stellmacher (1991) or Kuckein et al. (2009), as well as anything that can be learned from coronal field diagnostics in the microwave, IR and UV regimes (Section 3.5). Observations diagnosing the typical properties in or near the reconnection region, such as the field strength and connectivity, number density, temperature and velocity, are necessary input to impulsive phase 
reconnection and acceleration or heating models. Unfortunately the bulk properties readily observable with spectroscopic remote sensing may not be sufficient to understand the conditions that lead to what is in large part a plasma kinetic process (i.e., the decoupling of particles from the magnetic field and from each other). We should be prepared to learn what we can, in terms of the microphysics, from in situ observations in the solar wind or terrestrial environment.

Instability and field rearrangement: The evolution of a stable, energy-loaded configuration towards an unstable one, and the release of the stored energy, are related problems. Both require an understanding of the conditions under which magnetic reconnection will set in - or not - and this is a vast field of study in itself. One view is that the rate of magnetic reconnection is determined primarily by the plasma microphysical conditions. Another is that reconnection is determined by the magnetohydrodynamic state, and the microphysics somehow adapts to keep up with what the field dictates. But with neither field measurements nor detailed information on non-equilibrium plasma conditions in the corona where flare reconnection is thought to take place, we know little for sure about the reconnection physics. Observations of plasma inflow in the late phase of flares allows estimates to be made of the reconnection rate, which turns out to be compatible with fast reconnection (Yokoyama et al. 2001; Narukage \& Shibata 2006). But the rate alone is not very informative, and the late phase of a flare is a less challenging environment in terms of the reconnection and rate of energy conversion required.

The standard flare reconnection model has basically been in development since Giovanelli (1948). Note that Giovanelli's sketches of the magnetic scenarios for flares mainly emphasized the behavior of current systems, rather than flux transfer; as late as 1963 Parker (1963) could describe magnetic-field "annihilation" as a "presently popular belief" to explain solar flares, but that "There is very little in the observations to support such views." With Petschek (1964) augmenting the earlier work of Giovanelli, Cowling, Dungey, Sweet, and Parker himself, the case became much stronger. Nowadays it would be difficult to discuss flare phenomenology without appealing to magnetic reconnection in one form or another, although all of the abundant evidence for it is necessarily indirect. But once again, observational constraints on model geometry are few. On the disk, observationally-grounded extrapolations of the magnetic field are suggestive of one topology or another, sometimes backed up by the shape of the field outlined in EUV. For example, there is evidence for coronal nulls in flaring active regions (Aulanier et al. 2000; Fletcher et al. 2001; Masson et al. 2009). On the limb, double coronal sources are sometimes observed with a temperature structure consistent with outflows from a reconnecting structure, and a vertical displacement between the two sources suggestive of a vertically-extended current sheet (Sui \& Holman 2003). Though not strictly flare activity, the magnetic and EUV coronal evolution during a flux emergence event is consistent with a model involving separator reconnection (Longcope et al. 2005). During the impulsive phase, the portion of the field outlined by hot plasma can look very asymmetric and disordered, but later on in the EUV/SXR arcade it looks rather symmetric and quasi-2-D, like the standard cartoon. So all topologies are possible, and perhaps flare magnetic systems evolve through different topologies. However, returning to Parker's discussion of "annihilation," until we know whether we are dealing with reconnection or flux transfer at a neutral point, a neutral line, a separator, a current sheet or indeed a volume filled with many small current sheets it is not possible to say whether the energy released is dominated by field annihilation (i.e., dissipation of antiparallel components of $\mathbf{B}$ within the reconnecting structure) or field relaxation following reconnection ("dipolarization"). For example, reconnection at a null dissipates essentially no magnetic field because the recon- 
nection volume is so small - energy conversion happens elsewhere in the system - but this would not necessarily apply to a volume filled with many small current-sheet structures.

Energy conversion: The sudden transformation of energy contained in the solar magnetic field is the heart of the flare problem. As mentioned above, magnetic reconnection itself does not transform much energy, but may facilitate large-scale restructurings that do. The common ingredient in all flares, by any definition of their sudden appearance, is the impulsive phase. We identify this as the timing of the energetically fundamental non-thermal process of particle acceleration as originally observed at radio wavelengths, and then in Xrays and $\gamma$-rays as well. It is this impulsive phase, which we can associate with all of the dominant energetic processes of a flare (the white-light/UV continuum emission, large-scale wave generation, CME eruption, and powerful particle acceleration), that is not captured in the many cartoons showing different versions of the standard flare model.

The dominant models hold that particle acceleration is an entirely coronal process, with HXR footpoints produced by accelerated coronal electrons which precipitate, possibly having escaped a magnetic trap formed by the expansion of magnetic field into the corona. The presence of coronal hard X-ray sources (Krucker et al. 2008a) as well as radio bursts provides ample evidence that non-thermal electron populations are present in the corona during the flare impulsive phase, with high fractions of all electrons present being accelerated. In one case in particular, the data suggest that essentially all coronal electrons present in the source are non-thermal (Krucker et al. 2010), with mimimal remaining thermal distribution. Details of the acceleration mechanism are obscure, and observations in the optical to SXR regime which deal primarily with thermal (i.e., "processed" energy) are of limited help beyond suggesting the geometry and evolution of the environment in which acceleration occurs. Observational suggestions of a current sheet provide one possible environment, but if one is to produce the electron fluxes necessary to explain the HXR footpoints the required current sheet dimensions are rather unfeasible, unless the Alfvén speed is high (the number of electrons accelerated per second being limited to what can be advected into the sheet each second). We have evidence of magnetic field relaxation (Sui \& Holman 2003) consistent with shrinkage beneath a reconnection region, in which betatron acceleration would occur (Somov \& Kosugi 1997). But though this process can accelerate a good number of electrons, the energy increases achieved are modest, requiring a suprathermal population to start with (Giuliani et al. 2005; Karlický \& Bárta 2006). Non-thermal line broadening in soft X-ray (e.g., Ranns et al. 2000) and EUV lines (Imada et al. 2008; Hara et al. 2008) may be interpreted as evidence for plasma turbulence, a central ingredient in many electron acceleration models, though our observations are at a spatial scale far larger than that which plasma wave energy can be effectively dissipated by electron acceleration.

At some level, coronal electron acceleration is straightforward even though the details are unknown. A certain amount of energy dumped into the coronal plasma in the form of plasma turbulence, for example, must be shared between the coronal particles resulting in a mean energy per particle. If that mean energy is high enough that the electrons are collisionless on timescales of interest, a non-thermal distribution of some description must result. Even in the exceptional coronal source studied by Krucker et al. (2010) the numbers and energies are plausible. More challenging are the chromospheric HXR sources interpreted as due to escaping accelerated coronal electrons. The standard collisional thick-target electron beam model requires electron fluxes up to a few $\times 10^{35}$ electrons per second (Holman et al. 2003) leaving the corona. It is well known that this places strong demands on the coronal electron population, which amounts to perhaps $10^{36}$ in the volume above a flare region, and which would require replenishing during the course of the flare. Replenishing of the coro- 
nal acceleration volume by a counterstreaming return current generated by the plasma in which the electrons propagate (of equal and opposite electron flux) has been postulated, but it was pointed out relatively early on there would be problems with beam stability (Brown \& Melrose 1977), and this remains the case. White-light footpoint areas, which we might take as a proxy for the electron beam areas (HXR footpoint areas being hard to measure reliably) imply a beam flux and a return current speed in excess of what can propagate stably through the corona. At least according to our present theoretical understanding, the beam can travel stably only if the density of the loop in which it moves is large - on the order of $10^{11} \mathrm{~cm}^{-3}$ (see, e.g., van den Oord 1990, for details). Though this is possible later in the flare (once evaporation has started) there is little imaging or spectroscopic evidence for such loop densities before the flare (except in coronal thick target loop flares of Veronig \& Brown 2004, which do not show footpoints). Other exceptions might be found in the pre-flare phase of SOL2002-07-23T00:35 (X4.8), as discussed by Lin (2002) and Caspi \& Lin (2010). Despite this uncertainty the coronal electron beam model has been accepted for decades, but it is clear that renewed theoretical effort must be dedicated to understanding the propagation of a dense electron beam through the corona. Some alternatives to this model have also recently been proposed. Fletcher \& Hudson (2008) have introduced a model in which electrons producing the HXR footpoints are wholly accelerated in the chromosphere, and Brown et al. (2009) discuss the chromospheric re-acceleration of a small number of originally coronal electrons so that their photon yield per electron is increased, reducing the electron number and flux requirement. Overall, an instantaneous emission measure for the non-thermal electrons of as much as $10^{46} \mathrm{~cm}^{-3}$ is required to explain chromospheric HXR footpoints (Hoyng et al. 1976) which is achievable in the chromosphere, though still demanding. For example, if the flare footpoint area were around $10^{17} \mathrm{~cm}^{-2}$, then assuming a chromospheric slab of thickness $10^{8} \mathrm{~cm}$ would require on average $3 \times 10^{10}$ electrons $\mathrm{cm}^{-3}$ to be accelerated - on average about a tenth of the total electron population (bound or unbound) in the top $1000 \mathrm{~km}$ of the VAL-C chromospheric model (Vernazza et al. 1981).

Flares and CMEs: At present it seems clear that the standard flare scenario of large-scale reconnection in magnetic fields stretched by an eruption must explain a great deal of flare phenomenology. Probably Hirayama (1974) provided the first clear 3-D visualization of how this might feasibly happen, although the ideas certainly had been available long before this seminal paper appeared. In fact, the Hirayama work described a filament eruption (we would associate it with a CME nowadays), rather than a flare as such. Furthermore, we recognize that it is not the mass of the filament that is important in the overall dynamics, rather the evolution of the magnetic field which carries the filament mass along. The magnetic free energy released as the outwards ejection of mass is therefore at least as large as the CME kinetic energy estimated from the visible material. The CME is of course just another manifestation of the re-arrangement of coronal magnetic fields, but in an environment in which the perturbation can expand relatively freely into the corona above the reconnection region. This provides an interesting contrast to the situation below the reconnection region, where the magnetic rearrangement of strong fields releases a similar amount of energy into a small, confined volume of very low beta plasma, resulting primarily in non-thermal particles and heat, rather than mass motions. A basic calculation shows that $10^{31}$ erg released into a volume of $10^{27} \mathrm{~cm}^{3}$ at a mean density of say $10^{11} \mathrm{~cm}^{3}$ results in a mean energy per particle of $60 \mathrm{keV}$. Of course radiative and conductive losses reduce the instantaneous value, but it does make reasonably clear that flares are almost certain to be efficient producers of non-thermal populations. 
The rise of the active region filament heralding the imminent onset of a flare identifies the importance of the MHD instability - which will go on to produce the CME - in the whole flare/CME combination. But the extremely good correspondence in time between HXR bursts and both filament lift-off (Maričić et al. 2007; Temmer et al. 2008) and coronal "supra-arcade downflows" Asai et al. 2004b) (interpreted as retracting flux tubes formed in reconnection behind a CME) does not really permit us to say whether one is the "cause" and the other the "effect."

A further pioneering paper by Gold \& Hoyle (1960) had already described a large-scale reconnection scenario that did not require a prior eruption, and many flares, even including some as powerful as the low X class (Wang \& Zhang 2007) events, do not result from the open fields stretched out by a CME. Therefore models which permit the release of stored magnetic energy without field opening are also necessary - such as those proposing internal reconnections in active region core field (Gibson \& Fan 2006), or reconnection without full field opening (DeVore \& Antiochos 2008; Masson et al. 2009).

A final observational link which deserves modeling attention is the association of solar energetic particles with "soft-hard-harder" X-ray spectral evolution and fast CMEs (Grayson et al. 2009). The SHH evolution is clearly a property of a solar acceleration process which operates long after the CME has left, and long after the flare impulsive phase. It is apparently unique to flares exhibiting CMEs. Perhaps slow reconnection behind the departing CME, or dipolarization of reconnected fields which have been greatly stretched by the process, plays a part in the ongoing acceleration.

\subsection{Future observational progress}

Here we list a few important areas in which observations should be improved.

1. UV/EUV imaging spectroscopy. It is a major embarrassment to solar physics that we often turn to stellar observations to learn how to fill in missing "details" from the solar data. One such "detail" is the spectroscopy and morphology of Ly- $\alpha$ in flares (Rubio da Costa et al. 2009). Ly- $\alpha$ is a primary radiating component, rich in diagnostic information about the chromosphere. In general the visible/UV continuum contains the majority of flare radiated energy and yet we have few good observations of it (e.g., Neidig 1989).

2. Sensitive high-energy observations. RHESSI has made it abundantly clear that the key non-thermal processes involved in the disruption of coronal plasmas (i.e., flares and CMEs) can readily be detected even in the tenuous middle corona. There is a vast parameter space awaiting sensitive instruments.

3. Microwave/meter-wave imaging spectroscopy. Solar radio astronomy has not had the benefit yet of broadband observations in this key domain, or of radio imaging at more than a few frequencies. We know it to contain emission and absorption features of great diagnostic significance, as well as giving insight into the 3-D structure of the coronal magnetic field.

4. Neutral particle emissions. The detection of neutrons and energetic neutral atoms from solar flares is in its infancy but holds great promise for understanding the behavior of accelerated ions in the virtually unknown domain below a few $\mathrm{MeV}$. Neutron detectors placed at a few tenths of an AU will be of great value.

5. Coronal seismology. The wave population - background and transient - is another means whereby the coronal magnetic structure can be probed. This can be observed 
by high-resolution imaging and imaging spectroscopy of the global corona. The first steps in this area are now emerging from ground-based and Hinode observations, and the Solar Dynamics Observatory will provide a comprehensive imaging view because of its large telemetry bandwidth.

\section{Conclusions}

We have reviewed flare observations in a broad sense, touching on related phenomena and models that attempt to describe the overall process. The multifarious observations across the broad spectrum of phenomena each help us to characterize the equilibrium change in the corona and chromosphere that we call a flare, and it should be clear that the multiwavelength approach is crucial in flare studies. It tells us where the flare energy starts and where it ends up, and something about the intermediate steps. It also provides some geometrical and diagnostic information about the flare magnetic environment, at different levels in the atmosphere, and how and when this changes as the flare proceeds. This big picture cannot be reached using one spectral region on its own. The multiwavelength observations have many detailed applications as we try to understand specific mechanisms that are at work in various phases and regions of the flare development. Some of the mechanisms are at the stage of recent discovery and have only the sketchiest understanding at present. The coming decade will see a flood of multi-wavelength data, mastery of which which will require the development of new analysis techniques, such as fast image processing and feature recognition. It will be clear to the reader that much of the observational evidence presented here is based on the detailed analysis of small numbers of flares, and even basic statistical studies are rather few. But a comprehensive understanding of the flare phenomenon will require a blend of both approaches - i.e., the collection, sifting, comparison and assimilation of detailed properties of large samples of events. We look forward to the challenge.

Acknowledgements The authors would collectively like to acknowledge the work by the many instrument teams and software teams, whose sustained efforts over the years have made this kind of multiwavelength analysis possible. The chief architects of and major contributors to the Solarsoft library deserve particular thanks (Sam Freeland, Richard Schwartz, William Thompson, Kim Tolbert and Dominic Zarro). L.F. would like to acknowledge financial support from the UK STFC (Rolling grant ST/F0026371), the EU's SOLAIRE Research and Training Network (MTRN-CT-2006-035484) and L.F. and M.B. also acknowledge the support of the Leverhulme Foundation (Grant F/00 179/AY). The work of H. Ji was supported by NSFC 10833007 W. W. Liu was partly supported by an appointment to the NASA Postdoctoral Program at Goddard Space Flight Center, administered by Oak Ridge Associated Universities through a contract with NASA. Partial support to W. Liu's work was also provided by Hinode SOT contract NNM07AA01C. A. Caspi, H. Hudson, and S. Krucker were supported by NASA under contract NAS5-98033 and grants NAG5-12878 and NNX08AJ18G. A.V. and M.T. acknowledge the Austrian Science Fund (FWF) projects no. P20867-N16 and P20145-N16.

\section{References}

W. P. Abbett, S. L. Hawley, Astrophys. J. 521, 906 (1999), doi:10.1086/307576

J. Aboudarham, J. C. Henoux, Astron. Astrophys. 156, 73 (1986)

G.-X. Ai, Y.-F. Hu, Publications of the Beijing Astronomical Observatory 8, 1 (1986)

D. Alexander, A. J. Coyner, Astrophys. J. 640, 505 (2006), doi:10.1086/500076

D. Alexander, T. R. Metcalf, Solar Phys. 210, 323 (2002), doi:10.1023/A:1022457413628

J. C. Allred, S. L. Hawley, W. P. Abbett, M. Carlsson, Astrophys. J. 630, 573 (2005), arXiv: astro-ph/0507335 doi:10.1086/431751

E. Antonucci, B. R. Dennis, Solar Phys. 86, 67 (1983) 
B. Anwar, L. W. Acton, H. S. Hudson, M. Makita, A. N. McClymont, S. Tsuneta, Solar Phys. 147, 287 (1993), doi:10.1007/BF00690719

A. Asai, T. T. Ishii, H. Kurokawa, T. Yokoyama, M. Shimojo, Astrophys. J. 586, 624 (2003), doi: $10.1086 / 367694$

A. Asai, S. Masuda, T. Yokoyama, M. Shimojo, H. Isobe, H. Kurokawa, K. Shibata, Astrophys. J. Lett. 578, L91 (2002), arXiv: astro-ph/0209106 doi:10.1086/344566

A. Asai, H. Nakajima, M. Shimojo, S. M. White, H. S. Hudson, R. P. Lin, Pub. Astron. Soc. Japan 58, L1 (2006)

A. Asai, T. Yokoyama, M. Shimojo, S. Masuda, H. Kurokawa, K. Shibata, Astrophys. J. 611, 557 (2004a), doi:10.1086/422159

A. Asai, T. Yokoyama, M. Shimojo, K. Shibata, Astrophys. J. Lett. 605, L77 (2004b), doi:10.1086/420768

M. J. Aschwanden, Space Science Reviews 101, 1 (2002), doi:10.1023/A:1019712124366

M. J. Aschwanden, D. Alexander, Solar Phys. 204, 91 (2001), doi:10.1023/A:1014257826116

M. J. Aschwanden, A. O. Benz, B. R. Dennis, R. A. Schwartz, Astrophys. J. 455, 347 (1995a), doi: $10.1086 / 176582$

M. J. Aschwanden, R. A. Schwartz, D. M. Alt, Astrophys. J. 447, 923 (1995b), doi:10.1086/175930

M. J. Aschwanden, A. Winebarger, D. Tsiklauri, H. Peter, Astrophys. J. 659, 1673 (2007), doi: $10.1086 / 513070$

R. G. Athay, G. E. Moreton, Astrophys. J. 133, 935 (1961), doi:10.1086/147098

G. Aulanier, E. E. DeLuca, S. K. Antiochos, R. A. McMullen, L. Golub, Astrophys. J. 540, 1126 (2000), doi:10.1086/309376

G. Aulanier, E. Pariat, P. Démoulin, C. R. Devore, Solar Phys. 238, 347 (2006), doi:10.1007/s11207-0060230-2

Y. Avignon, J. Bonmartin, A. Bouteille, B. Clavelier, E. Hulot, Solar Phys. 120, 193 (1989)

T. Bai, Solar Phys. 62, 113 (1979), doi:10.1007/BF00150138

T. Bai, Astrophys. J. 308, 912 (1986), doi:10.1086/164561

G. Barnes, K. D. Leka, Astrophys. J. 646, 1303 (2006), doi:10.1086/504960

G. Barnes, D. W. Longcope, K. D. Leka, Astrophys. J. 629, 561 (2005), doi:10.1086/431175

T. S. Bastian, Advances in Space Research 32, 2705 (2003), doi:10.1016/S0273-1177(03)90454-6

T. S. Bastian, Planetary and Space Science 52, 1381 (2004), doi:10.1016/j.pss.2004.09.015

T. S. Bastian, A. O. Benz, D. E. Gary, ARAA 36, 131 (1998), doi:10.1146/annurev.astro.36.1.131

M. Battaglia, A. O. Benz, Astron. Astrophys. 456, 751 (2006), arXiv:astro-ph/0606353 doi:10.1051/0004-6361:20065233

M. Battaglia, L. Fletcher, A. O. Benz, Astron. Astrophys. 498, 891 (2009), 0903.2754 doi:10.1051/0004$6361 / 200811196$

P. J. Baum, A. Bratenahl, Solar Phys. 67, 245 (1980), doi:10.1007/BF00149805

A. O. Benz, ApJ 211, 270 (1977)

A. O. Benz, Living Reviews in Solar Physics 5, 1 (2008)

A. O. Benz, P. C. Grigis, A. Csillaghy, P. Saint-Hilaire, Solar Phys. 226, 121 (2005), astro-ph/0410436 doi:10.1007/s11207-005-5254-5

T. E. Berger, B. de Pontieu, C. J. Schrijver, A. M. Title, Astrophys. J. Lett. 519, L97 (1999), doi: $10.1086 / 312088$

D. A. Biesecker, D. C. Myers, B. J. Thompson, D. M. Hammer, A. Vourlidas, Astrophys. J. 569, 1009 (2002), doi:10.1086/339402

A. Bleybel, T. Amari, L. van Driel-Gesztelyi, K. D. Leka, Astron. Astrophys. 395, 685 (2002), doi:10.1051/0004-6361:20021332

S. A. Bogachev, B. V. Somov, T. Kosugi, T. Sakao, Astrophys. J. 630, 561 (2005), doi:10.1086/431918

L. Bone, J. C. Brown, L. Fletcher, A. Veronig, S. White, Astron. Astrophys. 446, 339 (2007), doi:10.1051/0004-6361:20020947

V. Bothmer, R. Schwenn, Annales Geophysicae 16, 1 (1998)

J. W. Brosius, Astrophys. J. 586, 1417 (2003), doi:10.1086/367958

J. W. Brosius, G. D. Holman, Astrophys. J. Lett. 659, L73 (2007), doi:10.1086/516629

J. W. Brosius, K. J. H. Phillips, Astrophys. J. 613, 580 (2004), doi:10.1086/422873

J. C. Brown, Solar Phys. 18, 489 (1971)

J. C. Brown, Solar Phys. 31, 143 (1973), doi:10.1007/BF00156080

J. C. Brown, A. J. Conway, M. J. Aschwanden, Astrophys. J. 509, 911 (1998), doi:10.1086/306522

J. C. Brown, P. C. V. Mallik, Astron. Astrophys. 481, 507 (2008), 0706.2823 doi:10.1051/00046361:20078103

J. C. Brown, P. C. V. Mallik, Astrophys. J. Lett. 697, L6 (2009), doi:10.1088/0004-637X/697/1/L6 
J. C. Brown, P. C. V. Mallik, N. R. Badnell, Astron. Astrophys. 515, C1+ (2010), doi:10.1051/00046361:20078103e

J. C. Brown, D. B. Melrose, Solar Phys. 52, 117 (1977), doi:10.1007/BF00935795

J. C. Brown, R. Turkmani, E. P. Kontar, A. L. MacKinnon, L. Vlahos, Astron. Astrophys. 508, 993 (2009), 0909.4243 doi:10.1051/0004-6361/200913145

G. E. Brueckner, R. A. Howard, M. J. Koomen, C. M. Korendyke, D. J. Michels, J. D. Moses, D. G. Socker, K. P. Dere, P. L. Lamy, A. Llebaria, M. V. Bout, R. Schwenn, G. M. Simnett, D. K. Bedford, C. J. Eyles, Solar Phys. 162, 357 (1995)

L. F. Burlaga, L. Klein, N. R. Sheeley, Jr., D. J. Michels, R. A. Howard, M. J. Koomen, R. Schwenn, H. Rosenbauer, Geophys. Res. Lett. 9, 1317 (1982), doi:10.1029/GL009i012p01317

R. Cameron, I. Sammis, Astrophys. J. Lett. 525, L61 (1999), doi:10.1086/312328

H. V. Cane, W. C. Erickson, Astrophys. J. 623, 1180 (2005), doi:10.1086/428820

R. C. Canfield, C.-C. Cheng, K. P. Dere, G. A. Dulk, D. J. McLean, E. J. Schmahl, R. D. Robinson, Jr., S. A. Schoolman, in Skylab Solar Workshop II, ed. by P. A. Sturrock (1980), pp. 451-469

R. C. Canfield, T. A. Gunkler, P. J. Ricchiazzi, Astrophys. J. 282, 296 (1984), doi:10.1086/162203

R. C. Canfield, T. R. Metcalf, K. T. Strong, D. M. Zarro, Nature 326, 165 (1987), doi:10.1038/326165a0

P. J. Cargill, J. T. Mariska, S. K. Antiochos, Astrophys. J. 439, 1034 (1995), doi:10.1086/175240

C. Caroubalos, Annales d'Astrophysique 27, 333 (1964)

R. C. Carrington, Mon. Not. Roy. Astron. Soc. 20, 13 (1859)

A. Caspi, Super-hot $(T>30 \mathrm{MK})$ Thermal Plasma in Solar Flares, Doctoral dissertation, University of California, Berkeley, Department of Physics (2010), URL http://sprg.ssl.berkeley.edu/ cepheid/.eprints/Caspi_Dissertation.pdf

A. Caspi, R. P. Lin, Astrophys. J. Lett. 725, L161 (2010), doi:10.1088/2041-8205/725/2/L161

C. P. Catalano, J. A. van Allen, Astrophys. J. 185, 335 (1973), doi:10.1086/152420

G. Cauzzi, K. P. Reardon, H. Uitenbroek, F. Cavallini, A. Falchi, R. Falciani, K. Janssen, T. Rimmele, A. Vecchio, F. Wöger, Astron. Astrophys. 480, 515 (2008), 0709.2417 doi:10.1051/0004-6361:20078642

Q. R. Chen, M. D. Ding, Astrophys. J. 618, 537 (2005), arXiv: astro-ph/0412171 doi:10.1086/425856

Q. R. Chen, M. D. Ding, Astrophys. J. 641, 1217 (2006), arXiv:astro-ph/0512496 doi:10.1086/500635

C. Chifor, D. Tripathi, H. E. Mason, B. R. Dennis, Astron. Astrophys. 472, 967 (2007), doi:10.1051/00046361:20077771

E. L. Chupp, D. J. Forrest, P. R. Higbie, A. N. Suri, C. Tsai, P. P. Dunphy, Nature 241, 333 (1973)

H. T. Claßen, G. Mann, A. Klassen, H. Aurass, Astron. Astrophys. 409, 309 (2003), doi:10.1051/00046361:20031097

E. W. Cliver, Solar Phys. 157, 285 (1995)

E. W. Cliver, Astrophys. J. 639, 1206 (2006), doi:10.1086/499765

E. W. Cliver, B. R. Dennis, A. L. Kiplinger, S. R. Kane, D. F. Neidig, N. R. Sheeley, M. J. Koomen, Astrophys. J. 305, 920 (1986)

E. W. Cliver, H. S. Hudson, Journal of Atmospheric and Solar-Terrestrial Physics 64, 231 (2002), doi:10.1016/S1364-6826(01)00086-4

E. W. Cliver, N. V. Nitta, B. J. Thompson, J. Zhang, Solar Phys. 225, 105 (2004), doi:10.1007/s11207-0043258-1

A. E. Covington, Solar Phys. 33, 439 (1973), doi:10.1007/BF00152431

A. N. Cox, Allen's astrophysical quantities (2000)

D. P. Cox, W. H. Tucker, Astrophys. J. 157, 1157 (1969)

N. B. Crosby, M. J. Aschwanden, B. R. Dennis, Solar Phys. 143, 275 (1993)

Y. Cui, R. Li, L. Zhang, Y. He, H. Wang, Solar Phys. 237, 45 (2006), doi:10.1007/s11207-006-0077-6

J. L. Culhane, Mon. Not. Roy. Astron. Soc. 144, 375 (1969)

J. L. Culhane, L. K. Harra, A. M. James, K. Al-Janabi, L. J. Bradley, R. A. Chaudry, K. Rees, J. A. Tandy, P. Thomas, M. C. R. Whillock, B. Winter, G. A. Doschek, C. M. Korendyke, C. M. Brown, S. Myers, J. Mariska, J. Seely, J. Lang, B. J. Kent, B. M. Shaughnessy, P. R. Young, G. M. Simnett, C. M. Castelli, S. Mahmoud, H. Mapson-Menard, B. J. Probyn, R. J. Thomas, J. Davila, K. Dere, D. Windt, J. Shea, R. Hagood, R. Moye, H. Hara, T. Watanabe, K. Matsuzaki, T. Kosugi, V. Hansteen, Ø. Wikstol, Solar Phys. 243, 19 (2007), doi:10.1007/s01007-007-0293-1

J. L. Culhane, A. T. Phillips, M. Inda-Koide, T. Kosugi, A. Fludra, H. Kurokawa, K. Makishima, C. D. Pike, T. Sakao, T. Sakurai, Solar Phys. 153, 307 (1994)

J. L. Culhane, J. F. Vesecky, K. J. H. Phillips, Solar Phys. 15, 394 (1970), doi:10.1007/BF00151847

A. Czaykowska, D. Alexander, B. De Pontieu, Astrophys. J. 552, 849 (2001), doi:10.1086/320553

A. Czaykowska, B. de Pontieu, D. Alexander, G. Rank, Astrophys. J. Lett. 521, L75 (1999), doi: $10.1086 / 312176$ 
B. De Pontieu, S. McIntosh, V. H. Hansteen, M. Carlsson, C. J. Schrijver, T. D. Tarbell, A. M. Title, R. A. Shine, Y. Suematsu, S. Tsuneta, Y. Katsukawa, K. Ichimoto, T. Shimizu, S. Nagata, Pub. Astron. Soc. Japan 59, 655 (2007), 0710.2934

C. Debi Prasad, Bulletin of the Astronomical Society of India 26, 253 (1998)

G. Del Zanna, A. Berlicki, B. Schmieder, H. E. Mason, Solar Phys. 234, 95 (2006), doi:10.1007/s11207-0060016-6

J.-P. Delaboudinière, G. E. Artzner, J. Brunaud, A. H. Gabriel, J. F. Hochedez, F. Millier, X. Y. Song, B. Au, K. P. Dere, R. A. Howard, R. Kreplin, D. J. Michels, J. D. Moses, J. M. Defise, C. Jamar, P. Rochus, J. P. Chauvineau, J. P. Marioge, R. C. Catura, J. R. Lemen, L. Shing, R. A. Stern, J. B. Gurman, W. M. Neupert, A. Maucherat, F. Clette, P. Cugnon, E. L. van Dessel, Solar Phys. 162, 291 (1995), doi:10.1007/BF00733432

P. Demoulin, L. G. Bagala, C. H. Mandrini, J. C. Henoux, M. G. Rovira, Astron. Astrophys. 325, 305 (1997)

P. Demoulin, J. C. Henoux, C. H. Mandrini, Solar Phys. 139, 105 (1992)

P. Demoulin, C. H. Mandrini, M. G. Rovira, J. C. Henoux, M. E. Machado, Solar Phys. 150, 221 (1994), doi:10.1007/BF00712887

P. Demoulin, L. van Driel-Gesztelyi, B. Schmieder, J. C. Hemoux, G. Csepura, M. J. Hagyard, Astron. Astrophys. 271, 292 (1993)

C. Denker, A. Tritschler, T. R. Rimmele, K. Richards, S. L. Hegwer, F. Wöger, Pub. Astron. Soc. Pac. 119, 170 (2007), doi:10.1086/512493

B. R. Dennis, R. L. Pernak, Astrophys. J. 698, 2131 (2009), doi:10.1088/0004-637X/698/2/2131

B. R. Dennis, D. M. Zarro, Solar Phys. 146, 177 (1993)

K. P. Dere, G. E. Brueckner, R. A. Howard, M. J. Koomen, C. M. Korendyke, R. W. Kreplin, D. J. Michels, J. D. Moses, N. E. Moulton, D. G. Socker, O. C. St. Cyr, J. P. Delaboudinière, G. E. Artzner, J. Brunaud, A. H. Gabriel, J. F. Hochedez, F. Millier, X. Y. Song, J. P. Chauvineau, J. P. Marioge, J. M. Defise, C. Jamar, P. Rochus, R. C. Catura, J. R. Lemen, J. B. Gurman, W. Neupert, F. Clette, P. Cugnon, E. L. van Dessel, P. L. Lamy, A. Llebaria, R. Schwenn, G. M. Simnett, Solar Phys. 175, 601 (1997a), doi:10.1023/A:1004907307376

K. P. Dere, E. Landi, H. E. Mason, B. C. Monsignori Fossi, P. R. Young, Astron. Astrophys. Suppl. 125, 149 (1997b)

A. Des Jardins, R. Canfield, D. Longcope, C. Fordyce, S. Waitukaitis, Astrophys. J. 693, 1628 (2009), doi:10.1088/0004-637X/693/2/1628

C. R. DeVore, S. K. Antiochos, Astrophys. J. 680, 740 (2008), doi:10.1086/588011

M. D. Ding, Q. R. Chen, J. P. Li, P. F. Chen, Astrophys. J. 598, 683 (2003), arXiv: astro-ph/0308085 doi: $10.1086 / 378877$

M. D. Ding, C. Fang, Astron. Astrophys. 314, 643 (1996)

H. W. Dodson, E. R. Hedeman, Solar Phys. 13, 401 (1970), doi:10.1007/BF00153560

A.-C. Donea, C. Lindsey, Astrophys. J. 630, 1168 (2005), doi:10.1086/432155

G. A. Doschek, U. Feldman, R. W. Kreplin, L. Cohen, Astrophys. J. 239, 725 (1980), doi:10.1086/158158

G. A. Doschek, J. T. Mariska, T. Sakao, Astrophys. J. 459, 823 (1996), doi:10.1086/176946

G. A. Doschek, H. P. Warren, Astrophys. J. 629, 1150 (2005), doi:10.1086/431920

W. Dröge, D. Ruffolo, B. Klecker, Astrophys. J. Lett. 464, L87+ (1996), arXiv:astro-ph/9604019 doi: $10.1086 / 310093$

M. A. Ellison, Mon. Not. Roy. Astron. Soc. 106, 500 (1946)

A. G. Emslie, B. R. Dennis, G. D. Holman, H. S. Hudson, Journal of Geophysical Research (Space Physics) 110, 11103 (2005), doi:10.1029/2005JA011305

A. G. Emslie, H. Kucharek, B. R. Dennis, N. Gopalswamy, G. D. Holman, G. H. Share, A. Vourlidas, T. G. Forbes, P. T. Gallagher, G. M. Mason, T. R. Metcalf, R. A. Mewaldt, R. J. Murphy, R. A. Schwartz, T. H. Zurbuchen, Journal of Geophysical Research (Space Physics) 109, 10104 (2004), doi:10.1029/2004JA010571

A. G. Emslie, K. J. H. Phillips, B. R. Dennis, Solar Phys. 103, 89 (1986)

A. G. Emslie, P. A. Sturrock, Solar Phys. 80, 99 (1982), doi:10.1007/BF00153426

P. Evenson, R. Kroeger, P. Meyer, D. Reames, Astrophys. Suppl. 73, 273 (1990), doi:10.1086/191462

P. Evenson, P. Meyer, K. R. Pyle, Astrophys. J. 274, 875 (1983), doi:10.1086/161500

D. A. Falconer, R. L. Moore, G. A. Gary, Astrophys. J. 569, 1016 (2002), doi:10.1086/339161

F. Fárník, H. Hudson, T. Watanabe, Solar Phys. 165, 169 (1996), doi:10.1007/BF00149096

F. Fárník, H. S. Hudson, M. Karlický, T. Kosugi, Astron. Astrophys. 399, 1159 (2003), doi:10.1051/00046361:20021852

F. Fárník, S. K. Savy, Solar Phys. 183, 339 (1998)

U. Feldman, Astrophys. J. 364, 322 (1990), doi:10.1086/169414

U. Feldman, I. Dammasch, E. Landi, G. A. Doschek, Astrophys. J. 609, 439 (2004), doi:10.1086/420964 
U. Feldman, J. M. Laming, Physica Scripta 61, 222 (2000)

G. H. Fisher, R. C. Canfield, A. N. McClymont, Astrophys. J. 289, 434 (1985a), doi:10.1086/162903

G. H. Fisher, R. C. Canfield, A. N. McClymont, Astrophys. J. 289, 425 (1985b), doi:10.1086/162902

G. H. Fisher, R. C. Canfield, A. N. McClymont, Astrophys. J. 289, 414 (1985c), doi:10.1086/162901

L. Fletcher, Astron. Astrophys. 493, 241 (2009), doi:10.1051/0004-6361:20077972

L. Fletcher, I. G. Hannah, H. S. Hudson, T. R. Metcalf, Astrophys. J. 656, 1187 (2007), doi:10.1086/510446

L. Fletcher, H. S. Hudson, Solar Phys. 210, 307 (2002), doi:10.1023/A:1022479610710

L. Fletcher, H. S. Hudson, Astrophys. J. 675, 1645 (2008), 0712.3452 doi:10.1086/527044

L. Fletcher, T. R. Metcalf, D. Alexander, D. S. Brown, L. A. Ryder, ApJ 554, 451 (2001)

L. Fletcher, J. A. Pollock, H. E. Potts, Solar Phys. 222, 279 (2004), doi:10.1023/B:SOLA.0000043580.89730.4d

T. G. Forbes, L. W. Acton, Astrophys. J. 459, 330 (1996), doi:10.1086/176896

T. G. Forbes, J. Lin, Journal of Atmospheric and Terrestrial Physics 62, 1499 (2000)

T. G. Forbes, J. M. Malherbe, E. R. Priest, Solar Phys. 120, 285 (1989)

C. Foullon, E. Verwichte, V. M. Nakariakov, L. Fletcher, Astron. Astrophys. 440, L59 (2005), doi:10.1051/0004-6361:200500169

K. J. Frost, B. R. Dennis, Astrophys. J. 165, 655 (1971)

Q. Fu, H. Ji, Z. Qin, Z. Xu, Z. Xia, H. Wu, Y. Liu, Y. Yan, G. Huang, Z. Chen, Z. Jin, Q. Yao, C. Cheng, F. Xu, M. Wang, L. Pei, S. Chen, G. Yang, C. Tan, S. Shi, Solar Phys. 222, 167 (2004), doi:10.1023/B:SOLA.0000036876.14446.dd

P. T. Gallagher, B. R. Dennis, S. Krucker, R. A. Schwartz, A. K. Tolbert, Solar Phys. 210, 341 (2002), doi:10.1023/A:1022422019779

P. T. Gallagher, G. R. Lawrence, B. R. Dennis, Astrophys. J. Lett. 588, L53 (2003), doi:10.1086/375504

D. E. Gary, G. J. Hurford, in Solar Physics with Radio Observations; Proc. Nobeyama Symp. (NRO Rep. 479), ed. by T. S. Bastian, N. Gopalswamy, K. Shibasaki (1999), p. 429

S. Gburek, J. Sylwester, P. Martens, Solar Phys. 239, 531 (2006), doi:10.1007/s11207-006-1994-0

M. K. Georgoulis, D. M. Rust, Astrophys. J. Lett. 661, L109 (2007), doi:10.1086/518718

S. E. Gibson, Y. Fan, Astrophys. J. Lett. 637, L65 (2006), doi:10.1086/500452

R. G. Giovanelli, Mon. Not. Roy. Astron. Soc. 108, 163 (1948)

P. Giuliani, T. Neukirch, P. Wood, Astrophys. J. 635, 636 (2005)

C. P. Goff, S. A. Matthews, L. van Driel-Gesztelyi, L. K. Harra, Astron. Astrophys. 423, 363 (2004), doi:10.1051/0004-6361:20040392

T. Gold, F. Hoyle, Mon. Not. Roy. Astron. Soc. 120, 89 (1960)

L. Golub, E. Deluca, G. Austin, J. Bookbinder, D. Caldwell, P. Cheimets, J. Cirtain, M. Cosmo, P. Reid, A. Sette, M. Weber, T. Sakao, R. Kano, K. Shibasaki, H. Hara, S. Tsuneta, K. Kumagai, T. Tamura, M. Shimojo, J. McCracken, J. Carpenter, H. Haight, R. Siler, E. Wright, J. Tucker, H. Rutledge, M. Barbera, G. Peres, S. Varisco, Solar Phys. 243, 63 (2007), doi:10.1007/s11207-007-0182-1

V. S. Gorbachev, B. V. Somov, Soviet Astronomy 33, 57 (1989)

J. D. Graham, A. Norton, A. López Ariste, B. Lites, H. Socas-Navarro, S. Tomczyk, in Astronomical Society of the Pacific Conference Series, ed. by J. Trujillo-Bueno \& J. Sanchez Almeida (2003), volume 307 of Astronomical Society of the Pacific Conference Series, pp. 131-+

J. A. Grayson, S. Krucker, R. P. Lin, Astrophys. J. 707, 1588 (2009), doi:10.1088/0004-637X/707/2/1588

P. C. Grigis, A. O. Benz, Astron. Astrophys. 426, 1093 (2004), astro-ph/0407431 doi:10.1051/00046361:20041367

P. C. Grigis, A. O. Benz, Astrophys. J. Lett. 625, L143 (2005), arXiv:astro-ph/0504436 doi: $10.1086 / 431147$

P. C. Grigis, A. O. Benz, Astrophys. J. 683, 1180 (2008), 0708.2472, doi:10.1086/589826

E. H. B. M. Gronenschild, R. Mewe, Astron. Astrophys. Suppl. 32, 283 (1978)

M. Gros, V. Tatischeff, J. Kiener, B. Cordier, C. Chapuis, G. Weidenspointner, G. Vedrenne, A. von Kienlin, R. Diehl, A. Bykov, M. NMéndez, in 5th INTEGRAL Workshop on the INTEGRAL Universe, ed. by V. Schoenfelder, G. Lichti, C. Winkler (2004), volume 552 of ESA Special Publication, pp. 669-+

M. J. Hagyard, N. P. Cumings, E. A. West, J. E. Smith, Solar Phys. 80, 33 (1982), doi:10.1007/BF00153422 M. J. Hagyard, D. Teuber, E. A. West, J. B. Smith, Solar Phys. 91, 115 (1984)

B. N. Handy, L. W. Acton, C. C. Kankelborg, C. J. Wolfson, D. J. Akin, M. E. Bruner, R. Caravalho, R. C. Catura, R. Chevalier, D. W. Duncan, C. G. Edwards, C. N. Feinstein, S. L. Freeland, F. M. Friedlaender, C. H. Hoffmann, N. E. Hurlburt, B. K. Jurcevich, N. L. Katz, G. A. Kelly, J. R. Lemen, M. Levay, R. W. Lindgren, D. P. Mathur, S. B. Meyer, S. J. Morrison, M. D. Morrison, R. W. Nightingale, T. P. Pope, R. A. Rehse, C. J. Schrijver, R. A. Shine, L. Shing, K. T. Strong, T. D. Tarbell, A. M. Title, D. D. Torgerson, L. Golub, J. A. Bookbinder, D. Caldwell, P. N. Cheimets, W. N. Davis, E. E. Deluca, R. A. McMullen, H. P. Warren, D. Amato, R. Fisher, H. Maldonado, C. Parkinson, Solar Phys. 187, 229 (1999) 
I. Hannah et al., Space Sci. Rev. pp. XXX-XXX (2011)

H. Hara, T. Watanabe, K. Matsuzaki, L. K. Harra, J. L. Culhane, P. Cargill, J. T. Mariska, G. A. Doschek, Pub. Astron. Soc. Japan 60, 275 (2008)

L. K. Harra, S. A. Matthews, J. L. Culhane, Astrophys. J. Lett. 549, L245 (2001), doi:10.1086/319163

L. K. Harra, D. R. Williams, A. J. Wallace, T. Magara, H. Hara, S. Tsuneta, A. C. Sterling, G. A. Doschek, Astrophys. J. Lett. 691, L99 (2009), doi:10.1088/0004-637X/691/2/L99

R. A. Harrison, E. C. Sawyer, M. K. Carter, A. M. Cruise, R. M. Cutler, A. Fludra, R. W. Hayes, B. J. Kent, J. Lang, D. J. Parker, J. Payne, C. D. Pike, S. C. Peskett, A. G. Richards, J. L. Culhane, K. Norman, A. A. Breeveld, E. R. Breeveld, K. F. A. Janabi, A. J. McCalden, J. H. Parkinson, D. G. Self, P. D. Thomas, A. I. Poland, R. J. Thomas, W. T. Thompson, O. Kjeldseth-Moe, P. Brekke, J. Karud, P. Maltby, B. Aschenbach, H. Brauninger, M. Kuhne, J. Hollandt, O. H. W. Siegmund, M. C. E. Huber, A. H. Gabriel, H. E. Mason, B. J. I. Bromage, Solar Phys. 162, 233 (1995)

R. A. Harrison, P. W. Waggett, R. D. Bentley, K. J. H. Phillips, M. Bruner, M. Dryer, G. M. Simnett, Solar Phys. 97, 387 (1985)

J. W. Harvey, F. Hill, R. Hubbard, J. R. Kennedy, J. W. Leibacher, J. A. Pintar, P. A. Gilman, R. W. Noyes, A. M. Title, J. Toomre, R. K. Ulrich, A. Bhatnagar, J. A. Kennewell, W. Marquette, J. Patrón, O. Saá, E. Yasukawa, Science 272, 1284 (1996), doi:10.1126/science.272.5266.1284

K. Harvey, N. Sheeley, Jr., J. Harvey, in Solar-Terrestrial Predictions, ed. by P. A. Simon, G. Heckman, M. A. Shea (1986), pp. 198-+

M. Hesse, T. G. Forbes, J. Birn, Astrophys. J. 631, 1227 (2005), doi:10.1086/432677

T. Hirayama, Solar Phys. 34, 323 (1974), doi:10.1007/BF00153671

G. D. Holman, L. Sui, R. A. Schwartz, A. G. Emslie, Astrophys. J. Lett. 595, L97 (2003), doi:10.1086/378488

G. Holman et al., Space Sci. Rev. pp. XXX-XXX (2011)

K. Hori, T. Yokoyama, T. Kosugi, K. Shibata, Astrophys. J. 500, 492 (1998), doi:10.1086/305725

R. A. Howard, J. D. Moses, A. Vourlidas, J. S. Newmark, D. G. Socker, S. P. Plunkett, C. M. Korendyke, J. W. Cook, A. Hurley, J. M. Davila, W. T. Thompson, O. C. St Cyr, E. Mentzell, K. Mehalick, J. R. Lemen, J. P. Wuelser, D. W. Duncan, T. D. Tarbell, C. J. Wolfson, A. Moore, R. A. Harrison, N. R. Waltham, J. Lang, C. J. Davis, C. J. Eyles, H. Mapson-Menard, G. M. Simnett, J. P. Halain, J. M. Defise, E. Mazy, P. Rochus, R. Mercier, M. F. Ravet, F. Delmotte, F. Auchere, J. P. Delaboudiniere, V. Bothmer, W. Deutsch, D. Wang, N. Rich, S. Cooper, V. Stephens, G. Maahs, R. Baugh, D. McMullin, T. Carter, Space Science Reviews 136, 67 (2008), doi:10.1007/s11214-008-9341-4

P. Hoyng, H. F. van Beek, J. C. Brown, Solar Phys. 48, 197 (1976)

X.-M. Hua, R. E. Lingenfelter, Solar Phys. 107, 351 (1987), doi:10.1007/BF00152031

H. Hudson, B. Haisch, K. T. Strong, J. Geophys. Res. 100, 3473 (1995), doi:10.1029/94JA02710

H. S. Hudson, Solar Phys. 24, 414 (1972)

H. S. Hudson, Solar Phys. 45, 69 (1975), doi:10.1007/BF00152217

H. S. Hudson, Astrophys. J. 224, 235 (1978), doi:10.1086/156370

H. S. Hudson, Astrophys. J. Lett. 531, L75 (2000), doi:10.1086/312516

H. S. Hudson, in The Physics of Chromospheric Plasmas, ed. by P. Heinzel, I. Dorotovič, R. J. Rutten (2007), volume 368 of Astronomical Society of the Pacific Conference Series, pp. 365-+

H. S. Hudson, L. W. Acton, S. L. Freeland, Astrophys. J. 470, 629 (1996), doi:10.1086/177894

H. S. Hudson, E. W. Cliver, J. Geophys. Res. 106, 25199 (2001), doi:10.1029/2000JA004026

H. S. Hudson, F. Fárník, in ESA SP-506: Solar Variability: From Core to Outer Frontiers, ed. by A. Wilson (2002), pp. 261-264

H. S. Hudson, G. H. Fisher, B. T. Welsch, in Subsurface and Atmospheric Influences on Solar Activity, ed. by R. Howe, R. W. Komm, K. S. Balasubramaniam, G. J. D. Petrie (2008), volume 383 of Astronomical Society of the Pacific Conference Series, pp. 221-+

H. S. Hudson, J. I. Khan, J. R. Lemen, N. V. Nitta, Y. Uchida, Solar Phys. 212, 121 (2003), doi:10.1023/A:1022904125479

H. S. Hudson, T. Kosugi, N. V. Nitta, M. Shimojo, Astrophys. J. Lett. 561, L211 (2001), doi:10.1086/324760

H. S. Hudson, R. P. Lin, R. T. Stewart, Solar Phys. 75, 245 (1982)

H. S. Hudson, K. Ohki, Solar Phys. 23, 155 (1972)

H. S. Hudson, K. T. Strong, B. R. Dennis, D. Zarro, M. Inda, T. Kosugi, T. Sakao, Astrophys. J. Lett. 422, L25 (1994), doi:10.1086/187203

H. S. Hudson, R. C. Willson, Solar Phys. 86, 123 (1983)

H. S. Hudson, C. J. Wolfson, T. R. Metcalf, Solar Phys. 234, 79 (2006), doi:10.1007/s11207-006-0056-y

G. J. Hurford, S. Krucker, R. P. Lin, R. A. Schwartz, G. H. Share, D. M. Smith, Astrophys. J. Lett. 644, L93 (2006), doi:10.1086/505329

G. J. Hurford, R. B. Read, H. Zirin, Solar Phys. 94, 413 (1984), doi:10.1007/BF00151327 
G. J. Hurford, E. J. Schmahl, R. A. Schwartz, A. J. Conway, M. J. Aschwanden, A. Csillaghy, B. R. Dennis, C. Johns-Krull, S. Krucker, R. P. Lin, J. McTiernan, T. R. Metcalf, J. Sato, D. M. Smith, Solar Phys. 210, 61 (2002), doi:10.1023/A:1022436213688

G. J. Hurford, R. A. Schwartz, S. Krucker, R. P. Lin, D. M. Smith, N. Vilmer, Astrophys. J. Lett. 595, L77 (2003), doi:10.1086/378179

S. Imada, H. Hara, T. Watanabe, A. Asai, T. Minoshima, L. K. Harra, J. T. Mariska, Astrophys. J. Lett. 679, L155 (2008), doi:10.1086/589444

A. R. Inglis, V. M. Nakariakov, Astron. Astrophys. 493, 259 (2009), doi:10.1051/0004-6361:200810473

H. Isobe, M. Kubo, T. Minoshima, K. Ichimoto, Y. Katsukawa, T. D. Tarbell, S. Tsuneta, T. E. Berger, B. Lites, S. Nagata, T. Shimizu, R. A. Shine, Y. Suematsu, A. M. Title, Pub. Astron. Soc. Japan 59, 807 (2007), 0711.3946

H. Isobe, T. Yokoyama, M. Shimojo, T. Morimoto, H. Kozu, S. Eto, N. Narukage, K. Shibata, Astrophys. J. 566, 528 (2002), doi:10.1086/324777

R. Jain, A. K. Pradhan, V. Joshi, K. J. Shah, J. J. Trivedi, S. L. Kayasth, V. M. Shah, M. R. Deshpande, Solar Phys. 239, 217 (2006), arXiv: astro-ph/0612356 doi:10.1007/s11207-006-0066-9

Å. M. Janse, B. C. Low, Astron. Astrophys. 472, 957 (2007), doi:10.1051/0004-6361:20077532

D. B. Jess, A. Andić, M. Mathioudakis, D. S. Bloomfield, F. P. Keenan, Astron. Astrophys. 473, 943 (2007), 0707.2716 doi:10.1051/0004-6361:20077142

D. B. Jess, M. Mathioudakis, P. J. Crockett, F. P. Keenan, Astrophys. J. Lett. 688, L119 (2008), 0810.1443 doi: $10.1086 / 595588$

H. Ji, G. Huang, H. Wang, Astrophys. J. 660, 893 (2007), doi:10.1086/513017

H. Ji, G. Huang, H. Wang, T. Zhou, Y. Li, Y. Zhang, M. Song, Astrophys. J. Lett. 636, L173 (2006), doi: $10.1086 / 500203$

H. Ji, H. Wang, P. R. Goode, Y. Jiang, V. Yurchyshyn, Astrophys. J. Lett. 607, L55 (2004), doi: $10.1086 / 421550$

H. Ji, H. Wang, C. Liu, B. R. Dennis, Astrophys. J. 680, 734 (2008), doi:10.1086/587138

M. Jin, M. Ding, Pub. Astron. Soc. Japan 60, 835 (2008)

J. Jing, J. Lee, C. Liu, D. E. Gary, H. Wang, Astrophys. J. Lett. 664, L127 (2007), doi:10.1086/520812

J. Jing, J. Qiu, J. Lin, M. Qu, Y. Xu, H. Wang, Astrophys. J. 620, 1085 (2005), doi:10.1086/427165

J. Jing, T. Wiegelmann, Y. Suematsu, M. Kubo, H. Wang, Astrophys. J. Lett. 676, L81 (2008), doi:10.1086/587058

K. Jiricka, M. Karlicky, O. Kepka, A. Tlamicha, Solar Phys. 147, 203 (1993), doi:10.1007/BF00675495

B. Joshi, P. K. Manoharan, A. M. Veronig, P. Pant, K. Pandey, Solar Phys. 242, 143 (2007), arXiv:astro-ph/0701368 doi:10.1007/s11207-007-0275-X

S. Kahler, Astrophys. J. 214, 891 (1977)

S. Kahler, Astrophys. J. 428, 837 (1994), doi:10.1086/174292

M. L. Kaiser, T. A. Kucera, J. M. Davila, O. C. St. Cyr, M. Guhathakurta, E. Christian, Space Science Reviews pp. 198-+ (2007), doi:10.1007/s11214-007-9277-0

S. R. Kane, Astrophys. J. Lett. 157, L139+ (1969), doi:10.1086/180403

S. R. Kane, K. A. Anderson, W. D. Evans, R. W. Klebesadel, J. Laros, Astrophys. J. Lett. 233, L151 (1979a), doi:10.1086/183095

S. R. Kane, R. F. Donnelly, Astrophys. J. 164, 151 (1971)

S. R. Kane, K. J. Frost, R. F. Donnelly, Astrophys. J. 234, 669 (1979b), doi:10.1086/157544

S. R. Kane, J. McTiernan, J. Loran, E. E. Fenimore, R. W. Klebesadel, J. G. Laros, Astrophys. J. 390, 687 (1992), doi:10.1086/171320

M. Karlický, M. Bárta, Astrophys. J. 647, 1472 (2006), doi:10.1086/505460

M. Karlický, T. Kosugi, Astron. Astrophys. 419, 1159 (2004), doi:10.1051/0004-6361:20034323

P. Kaufmann, in Energy Conversion and Particle Acceleration in the Solar Corona, ed. by L. Klein (2003), volume 612 of Lecture Notes in Physics, Berlin Springer Verlag, pp. 294-313

C. U. Keller, J. W. Harvey, M. S. Giampapa, in Society of Photo-Optical Instrumentation Engineers (SPIE) Conference Series, ed. by S. L. Keil, S. V. Avakyan (2003), volume 4853 of Society of Photo-Optical Instrumentation Engineers (SPIE) Conference Series, pp. 194-204

J. I. Khan, H. Aurass, Astron. Astrophys. 383, 1018 (2002), doi:10.1051/0004-6361:20011707

J. I. Khan, H. M. Bain, L. Fletcher, Astron. Astrophys. 475, 333 (2007), doi:10.1051/0004-6361:20077894

A. L. Kiplinger, Astrophys. J. 453, 973 (1995), doi:10.1086/176457

T. Kondo, T. Isobe, S. Igi, S.-i. Watari, M. Tokumaru, Communications Research Laboratory Review 40, 85 (1994)

E. P. Kontar, I. G. Hannah, A. L. MacKinnon, Astron. Astrophys. 489, L57 (2008), 0808.3334 doi:10.1051/0004-6361:200810719

R. A. Kopp, G. W. Pneuman, Solar Phys. 50, 85 (1976) 
H. Koshiishi, S. Enome, H. Nakajima, K. Shibasaki, M. Nishio, T. Takano, Y. Hanaoka, C. Torii, H. Sekiguchi, S. Kawashima, T. Bushimata, N. Shinohara, Y. Irimajiri, Y. Shiomi, Pub. Astron. Soc. Japan 46, L33 (1994)

A. G. Kosovichev, Solar Phys. 238, 1 (2006), arXiv : astro-ph/0601006 doi:10.1007/s11207-006-0190-6

A. G. Kosovichev, Astrophys. J. Lett. 670, L65 (2007), 0710.0757. doi:10.1086/524036

A. G. Kosovichev, V. V. Zharkova, Nature 393, 317 (1998), doi:10.1038/30629

N. D. Kostiuk, S. B. Pikelner, Soviet Astronomy 18, 590 (1975)

T. Kosugi, S. Masuda, K. Makishima, M. Inda, T. Murakami, T. Dotani, Y. Ogawara, T. Sakao, K. Kai, H. Nakajima, Solar Phys. 136, 17 (1991)

M. Kretzschmar, in 37th COSPAR Scientific Assembly (2008), volume 37 of COSPAR, Plenary Meeting, pp. $1617-+$

S. Krucker, M. Battaglia, P. J. Cargill, L. Fletcher, H. S. Hudson, A. L. MacKinnon, S. Masuda, L. Sui, M. Tomczak, A. L. Veronig, L. Vlahos, S. M. White, Astron. Astrophys. Rev. 16, 155 (2008a), doi:10.1007/s00159-008-0014-9

S. Krucker, M. D. Fivian, R. P. Lin, Advances in Space Research 35, 1707 (2005), doi:10.1016/j.asr.2005.05.054

S. Krucker, H. S. Hudson, L. Glesener, S. M. White, S. Masuda, J. Wuelser, R. P. Lin, Astrophys. J. 714, 1108 (2010), doi:10.1088/0004-637X/714/2/1108

S. Krucker, G. J. Hurford, R. P. Lin, Astrophys. J. Lett. 595, L103 (2003), doi:10.1086/378840

S. Krucker, G. J. Hurford, A. L. MacKinnon, A. Y. Shih, R. P. Lin, Astrophys. J. Lett. 678, L63 (2008b), doi:10.1086/588381

S. Krucker, S. M. White, R. P. Lin, Astrophys. J. Lett. 669, L49 (2007), doi:10.1086/523759

C. Kuckein, R. Centeno, V. Martínez Pillet, R. Casini, R. Manso Sainz, T. Shimizu, Astron. Astrophys. 501, 1113 (2009), 0904.4876 doi:10.1051/0004-6361/200911800

M. R. Kundu, Solar radio astronomy (Interscience, 1965)

M. R. Kundu, N. Nitta, S. M. White, K. Shibasaki, S. Enome, T. Sakao, T. Kosugi, T. Sakurai, Astrophys. J. 454, 522 (1995), doi:10.1086/176503

J. Lang, R. D. Bentley, C. M. Brown, J. L. Culhane, G. A. Doschek, T. Watanabe, E. Hiei, R. D. Deslattes, A. Fludra, P. R. Guttridge, J. E. Magraw, J. Payne, C. D. Pike, M. W. Trow, Pub. Astron. Soc. Japan 44, L55 (1992)

J. Lee, D. E. Gary, G. S. Choe, Astrophys. J. 647, 638 (2006), doi:10.1086/505416

K. D. Leka, G. Barnes, Astrophys. J. 595, 1277 (2003), doi:10.1086/377511

K. D. Leka, G. Barnes, Astrophys. J. 656, 1173 (2007), doi:10.1086/510282

P. Lemaire, P. Gouttebroze, J.-C. Vial, W. Curdt, U. Schühle, K. Wilhelm, Astron. Astrophys. 418, 737 (2004), doi:10.1051/0004-6361:20034405

H. Li, J.-Q. You, Q.-D. Wu, X.-F. Yu, Chinese Physics Letters 19, 742 (2002), doi:10.1088/0256$307 \mathrm{X} / 19 / 5 / 342$

J. Li, T. R. Metcalf, R. C. Canfield, J.-P. Wuelser, T. Kosugi, Astrophys. J. 482, 490 (1997), doi:10.1086/304131

P. Li, A. G. Emslie, J. T. Mariska, Astrophys. J. 417, 313 (1993), doi:10.1086/173314

Y. P. Li, W. Q. Gan, Astrophys. J. Lett. 629, L137 (2005), doi:10.1086/444617

Y. P. Li, W. Q. Gan, Astrophys. J. Lett. 652, L61 (2006), doi:10.1086/509879

Y. P. Li, W. Q. Gan, Advances in Space Research 39, 1389 (2007), doi:10.1016/j.asr.2007.04.009

Y. P. Li, W. Q. Gan, Solar Phys. 247, 77 (2008), doi:10.1007/s11207-007-9092-5

A. C. Lin, R. W. Nightingale, T. D. Tarbell, Solar Phys. 198, 385 (2001), doi:10.1023/A:1005213527766

H. Lin, J. R. Kuhn, R. Coulter, Astrophys. J. Lett. 613, L177 (2004), doi:10.1086/425217

H. Lin, M. J. Penn, S. Tomczyk, Astrophys. J. Lett. 541, L83 (2000), doi:10.1086/312900

R. P. Lin, Solar Phys. 15, 453 (1970)

R. P. Lin, B. R. Dennis, G. J. Hurford, D. M. Smith, A. Zehnder, P. R. Harvey, D. W. Curtis, D. Pankow, P. Turin, M. Bester, A. Csillaghy, M. Lewis, N. Madden, H. F. van Beek, M. Appleby, T. Raudorf, J. McTiernan, R. Ramaty, E. Schmahl, R. Schwartz, S. Krucker, R. Abiad, T. Quinn, P. Berg, M. Hashii, R. Sterling, R. Jackson, R. Pratt, R. D. Campbell, D. Malone, D. Landis, C. P. Barrington-Leigh, S. Slassi-Sennou, C. Cork, D. Clark, D. Amato, L. Orwig, R. Boyle, I. S. Banks, K. Shirey, A. K. Tolbert, D. Zarro, F. Snow, K. Thomsen, R. Henneck, A. McHedlishvili, P. Ming, M. Fivian, J. Jordan, R. Wanner, J. Crubb, J. Preble, M. Matranga, A. Benz, H. Hudson, R. C. Canfield, G. D. Holman, C. Crannell, T. Kosugi, A. G. Emslie, N. Vilmer, J. C. Brown, C. Johns-Krull, M. Aschwanden, T. Metcalf, A. Conway, Solar Phys. 210, 3 (2002), doi:10.1023/A:1022428818870

R. P. Lin, H. S. Hudson, Solar Phys. 50, 153 (1976)

R. P. Lin, S. Krucker, G. J. Hurford, D. M. Smith, H. S. Hudson, G. D. Holman, R. A. Schwartz, B. R. Dennis, G. H. Share, R. J. Murphy, A. G. Emslie, C. Johns-Krull, N. Vilmer, Astrophys. J. Lett. 595, L69 (2003), 
doi: $10.1086 / 378932$

R. P. et al. Lin, Solar Phys. 210 (2002)

C. Lindsey, A.-C. Donea, Solar Phys. 251, 627 (2008), doi:10.1007/s11207-008-9140-9

B. W. Lites, D. F. Elmore, K. V. Streander, in Advanced Solar Polarimetry - Theory, Observation, and Instrumentation, ed. by M. Sigwarth (2001), volume 236 of Astronomical Society of the Pacific Conference Series, pp. 33-+

C. Liu, N. Deng, Y. Liu, D. Falconer, P. R. Goode, C. Denker, H. Wang, Astrophys. J. 622, 722 (2005), doi: $10.1086 / 427868$

C. Liu, J. Lee, D. E. Gary, H. Wang, Astrophys. J. Lett. 658, L127 (2007), arXiv:astro-ph/0702326 doi:10.1086/513739

C. Liu, J. Lee, J. Jing, D. E. Gary, H. Wang, Astrophys. J. Lett. 672, L69 (2008a), 0711.1370 doi: $10.1086 / 525849$

R. Liu, H. Wang, D. Alexander, Astrophys. J. 696, 121 (2009a), doi:10.1088/0004-637X/696/1/121

W. Liu, Solar Flares as Natural Particle Accelerators: A High-energy View from X-ray Observations and Theoretical Models (VDM Verlag Dr, 2008)

W. Liu, Y. W. Jiang, S. Liu, V. Petrosian, Astrophys. J. Lett. 611, L53 (2004), arXiv: astro-ph/0401381 doi:10.1086/423371

W. Liu, S. Liu, Y. W. Jiang, V. Petrosian, Astrophys. J. 649, 1124 (2006), arXiv:astro-ph/0603510 doi: $10.1086 / 506268$

W. Liu, V. Petrosian, B. R. Dennis, G. D. Holman, Astrophys. J. 693, 847 (2009b), 0805.1055 doi:10.1088/0004-637X/693/1/847

W. Liu, V. Petrosian, B. R. Dennis, Y. W. Jiang, Astrophys. J. 676, 704 (2008b), 0709.1963 doi: $10.1086 / 527538$

W. Liu, V. Petrosian, J. T. Mariska, ArXiv e-prints (2009c), 0906.2449

W. Liu, T.-J. Wang, B. R. Dennis, G. D. Holman, Astrophys. J. 698, 632 (2009d), 0902.1805 doi:10.1088/0004-637X/698/1/632

D. W. Longcope, Solar Phys. 169, 91 (1996), doi:10.1007/BF00153836

D. W. Longcope, Living Reviews in Solar Physics 2, 7 (2005)

D. W. Longcope, D. E. McKenzie, J. Cirtain, J. Scott, Astrophys. J. 630, 596 (2005), doi:10.1086/432039

W. J. MacCombie, D. M. Rust, Solar Phys. 61, 69 (1979), doi:10.1007/BF00155447

M. E. Machado, E. H. Avrett, R. Falciani, C. Fang, L. Gesztelyi, J.-C. Henoux, E. Hiei, D. F. Neidig, D. M. Rust, P. Sotirovski, Z. Svestka, H. Zirin, in The lower atmosphere of solar flares (1986), pp. 483-488

M. E. Machado, E. H. Avrett, J. E. Vernazza, R. W. Noyes, Astrophys. J. 242, 336 (1980), doi:10.1086/158467

M. E. Machado, A. G. Emslie, J. C. Brown, Solar Phys. 58, 363 (1978), doi:10.1007/BF00157282

C. H. Mandrini, P. Demoulin, J. C. Henoux, M. E. Machado, Astron. Astrophys. 250, 541 (1991)

C. H. Mandrini, P. Demoulin, M. G. Rovira, J.-F. de La Beaujardiere, J. C. Henoux, Astron. Astrophys. 303, 927 (1995)

G. Mann, H. Aurass, W. Voigt, J. Paschke, in Coronal Streamers, Coronal Loops, and Coronal and Solar Wind Composition, Proceedings of the First SOHO Workshop, ESA SP-348, ed. by V. Domingo, A. Poland, J. Mariska (1992), pp. 129-+

G. Mann, A. Klassen, H. Aurass, H.-T. Classen, Astron. Astrophys. 400, 329 (2003), doi:10.1051/00046361:20021593

D. Maričić, B. Vršnak, A. L. Stanger, A. M. Veronig, M. Temmer, D. Roša, Solar Phys. 241, 99 (2007), doi:10.1007/s 11207-007-0291-X

S. F. Martin, Solar Phys. 68, 217 (1980)

J. C. Martínez-Oliveros, A.-C. Donea, Mon. Not. Roy. Astron. Soc. 395, L39 (2009), 0902.3856 doi:10.1111/j.1745-3933.2009.00637.x

J. C. Martínez-Oliveros, A.-C. Donea, P. S. Cally, H. Moradi, Mon. Not. Roy. Astron. Soc. 389, 1905 (2008), 0807.3783 doi:10.1111/j.1365-2966.2008.13722.x

S. Masson, E. Pariat, G. Aulanier, C. J. Schrijver, Astrophys. J. 700, 559 (2009), doi:10.1088/0004$637 \mathrm{X} / 700 / 1 / 559$

S. Masuda, T. Kosugi, H. Hara, S. Tsuneta, Y. Ogawara, Nature 371, 495 (1994), doi:10.1038/371495a0

S. Masuda, T. Kosugi, H. S. Hudson, Solar Phys. 204, 55 (2001)

S. A. Matthews, L. van Driel-Gesztelyi, H. S. Hudson, N. V. Nitta, Astron. Astrophys. 409, 1107 (2003), doi:10.1051/0004-6361:20031187

P. Mazzotta, G. Mazzitelli, S. Colafrancesco, N. Vittorio, Astron. Astrophys. Suppl. 133, 403 (1998), arXiv: astro-ph/9806391 doi:10.1051/aas:1998330

R. T. J. McAteer, P. T. Gallagher, J. Ireland, Astrophys. J. 631, 628 (2005), doi:10.1086/432412

K. G. McClements, D. Alexander, Astrophys. J. 619, 1153 (2005), doi:10.1086/426581 
P. S. McIntosh, Solar Phys. 125, 251 (1990)

P. S. McIntosh, R. F. Donnelly, Solar Phys. 23, 444 (1972), doi:10.1007/BF00148107

D. E. McKenzie, H. S. Hudson, Astrophys. J. Lett. 519, L93 (1999), doi:10.1086/312110

J. M. McTiernan, G. H. Fisher, P. Li, Astrophys. J. 514, 472 (1999), doi:10.1086/306924

J. M. McTiernan, S. R. Kane, J. M. Loran, J. R. Lemen, L. W. Acton, H. Hara, S. Tsuneta, T. Kosugi, Astrophys. J. Lett. 416, L91+ (1993), doi:10.1086/187078

P. Mein, Astron. Astrophys. 381, 271 (2002), doi:10.1051/0004-6361:20011418

A. M. Melo, P. Kaufmann, A. S. Kudaka, J.-P. Raulin, R. Marcon, A. Marun, P. Pereyra, H. Levato, Pub. Astron. Soc. Pac. 118, 1558 (2006), doi:10.1086/509267

P. Messmer, A. O. Benz, C. Monstein, Solar Phys. 187, 335 (1999), doi:10.1023/A:1005194314845

T. R. Metcalf, D. Alexander, H. S. Hudson, D. W. Longcope, Astrophys. J. 595, 483 (2003), doi: $10.1086 / 377217$

T. R. Metcalf, R. C. Canfield, E. H. Avrett, F. T. Metcalf, Astrophys. J. 350, 463 (1990), doi:10.1086/168400

T. R. Metcalf, H. S. Hudson, T. Kosugi, R. C. Puetter, R. K. Pina, Astrophys. J. 466, 585 (1996), doi:10.1086/177533

T. R. Metcalf, L. Jiao, A. N. McClymont, R. C. Canfield, H. Uitenbroek, Astrophys. J. 439, 474 (1995), doi: $10.1086 / 175188$

T. R. Metcalf, K. D. Leka, D. L. Mickey, Astrophys. J. Lett. 623, L53 (2005), doi:10.1086/429961

T. R. Metcalf, D. L. Mickey, B. J. Labonte, L. A. Ryder, in Multi-Wavelength Observations of Coronal Structure and Dynamics, ed. by P. C. H. Martens, D. Cauffman (2002), pp. 249-+

R. A. Mewaldt, C. M. S. Cohen, J. Giacalone, G. M. Mason, E. E. Chollet, M. I. Desai, D. K. Haggerty, M. D. Looper, R. S. Selesnick, A. Vourlidas, in American Institute of Physics Conference Series, ed. by G. Li, Q. Hu, O. Verkhoglyadova, G. P. Zank, R. P. Lin, \& J. Luhmann (2008), volume 1039 of American Institute of Physics Conference Series, pp. 111-117, doi:10.1063/1.2982431

R. A. Mewaldt, R. A. Leske, E. C. Stone, A. F. Barghouty, A. W. Labrador, C. M. S. Cohen, A. C. Cummings, A. J. Davis, T. T. von Rosenvinge, M. E. Wiedenbeck, Astrophys. J. Lett. 693, L11 (2009), doi:10.1088/0004-637X/693/1/L11

J.-P. Meyer, Astrophys. Suppl. 57, 173 (1985), doi:10.1086/191001

D. L. Mickey, R. C. Canfield, B. J. Labonte, K. D. Leka, M. F. Waterson, H. M. Weber, Solar Phys. 168, 229 (1996), doi:10.1007/BF00148052

C. H. Miklenic, A. M. Veronig, B. Vršnak, A. Hanslmeier, Astron. Astrophys. 461, 697 (2007), doi:10.1051/0004-6361:20065751

J. A. Miller, T. N. Larosa, R. L. Moore, Astrophys. J. 461, 445 (1996), doi:10.1086/177072

R. O. Milligan, Astrophys. J. Lett. 680, L157 (2008), doi:10.1086/589856

R. O. Milligan, B. R. Dennis, Astrophys. J. 699, 968 (2009), 0905.1669 doi:10.1088/0004-637X/699/2/968

R. O. Milligan, P. T. Gallagher, M. Mathioudakis, D. S. Bloomfield, F. P. Keenan, R. A. Schwartz, Astrophys. J. Lett. 638, L117 (2006a), arXiv:astro-ph/0509664 doi:10.1086/500555

R. O. Milligan, P. T. Gallagher, M. Mathioudakis, F. P. Keenan, Astrophys. J. Lett. 642, L169 (2006b), arXiv:astro-ph/0603652 doi:10.1086/504592

T. G. Moran, D. E. Jennings, L. D. Deming, G. H. McCabe, P. V. Sada, R. J. Boyle, Solar Phys. 241, 213 (2007), doi:10.1007/s11207-007-0271-1

D. Moses, F. Clette, J.-P. Delaboudinière, G. E. Artzner, M. Bougnet, J. Brunaud, C. Carabetian, A. H. Gabriel, J. F. Hochedez, F. Millier, X. Y. Song, B. Au, K. P. Dere, R. A. Howard, R. Kreplin, D. J. Michels, J. M. Defise, C. Jamar, P. Rochus, J. P. Chauvineau, J. P. Marioge, R. C. Catura, J. R. Lemen, L. Shing, R. A. Stern, J. B. Gurman, W. M. Neupert, J. Newmark, B. Thompson, A. Maucherat, F. PortierFozzani, D. Berghmans, P. Cugnon, E. L. van Dessel, J. R. Gabryl, Solar Phys. 175, 571 (1997), doi:10.1023/A:1004902913117

T. Mrozek, M. Tomczak, Astron. Astrophys. 415, 377 (2004), doi:10.1051/0004-6361:20034598

T. Mrozek, M. Tomczak, S. Gburek, Astron. Astrophys. 472, 945 (2007), doi:10.1051/0004-6361:20077652

Y. Muraki, H. Tsuchiya, K. Fujiki, S. Masuda, Y. Matsubara, H. Menjyo, T. Sako, K. Watanabe, M. Ohnishi,

A. Shiomi, M. Takita, T. Yuda, Y. Katayose, N. Hotta, S. Ozawa, T. Sakurai, Y. H. Tan, J. L. Zhang, Astroparticle Physics 28, 119 (2007), doi:10.1016/j.astropartphys.2007.04.012

N. Narukage, K. Shibata, Astrophys. J. 637, 1122 (2006), doi:10.1086/498492

D. F. Neidig, Solar Phys. 121, 261 (1989), doi:10.1007/BF00161699

W. M. Neupert, ApJ 153, L59 (1968)

Z. Ning, Solar Phys. 248, 99 (2008), doi:10.1007/s11207-008-9124-9

L. Ofman, L. Sui, Astrophys. J. Lett. 644, L149 (2006), doi:10.1086/505622

K. Ohki, T. Takakura, S. Tsuneta, N. Nitta, Solar Phys. 86, 301 (1983)

V. Ontiveros, A. Vourlidas, Astrophys. J. 693, 267 (2009), 0811.3743. doi:10.1088/0004-637X/693/1/267

F. Q. Orrall, J. B. Zirker, Astrophys. J. 208, 618 (1976), doi:10.1086/154642 
L. E. Orwig, K. J. Frost, B. R. Dennis, Solar Phys. 65, 25 (1980), doi:10.1007/BF00151382

W. Otruba, W. Pötzi, Hvar Observatory Bulletin 27, 189 (2003)

R. Pallavicini, S. Serio, G. S. Vaiana, Astrophys. J. 216, 108 (1977)

R. Pallavicini, G. S. Vaiana, S. W. Kahler, A. S. Krieger, Solar Phys. 45, 411 (1975)

I. D. Palmer, S. F. Smerd, Solar Phys. 26, 460 (1972)

E. N. Parker, Astrophys. Suppl. 8, 177 (1963), doi:10.1086/190087

G. K. Parks, J. R. Winckler, ApJ 155, L117 (1969)

A. N. Parmar, J. L. Culhane, C. G. Rapley, C. J. Wolfson, L. W. Acton, K. J. H. Phillips, B. R. Dennis, Astrophys. J. 279, 866 (1984), doi:10.1086/161957

M. R. Perrenoud, Solar Phys. 81, 197 (1982), doi:10.1007/BF00151991

V. Petrosian, S. Liu, Astrophys. J. 610, 550 (2004), arXiv: astro-ph/0401585 doi:10.1086/421486

H. E. Petschek, in The Physics of Solar Flares, ed. by W. N. Hess (1964), pp. 425-+

K. J. H. Phillips, Astrophys. J. 605, 921 (2004), doi:10.1086/382523

K. J. H. Phillips, C. Chifor, B. R. Dennis, Astrophys. J. 647, 1480 (2006), arXiv:astro-ph/0607309 doi:10.1086/505518

K. J. H. Phillips, U. Feldman, L. K. Harra, Astrophys. J. 634, 641 (2005), doi:10.1086/491693

K. J. H. Phillips, W. M. Neupert, Solar Phys. 32, 209 (1973), doi:10.1007/BF00152737

M. Pick, T. G. Forbes, G. Mann, H. V. Cane, J. Chen, A. Ciaravella, H. Cremades, R. A. Howard, H. S. Hudson, A. Klassen, K. L. Klein, M. A. Lee, J. A. Linker, D. Maia, Z. Mikic, J. C. Raymond, M. J. Reiner, G. M. Simnett, N. Srivastava, D. Tripathi, R. Vainio, A. Vourlidas, J. Zhang, T. H. Zurbuchen, N. R. Sheeley, C. Marqué, Space Science Reviews 123, 341 (2006), doi:10.1007/s11214-006-9021-1

A. I. Poland, R. A. Howard, M. J. Koomen, D. J. Michels, N. R. Sheeley, Jr., Solar Phys. 69, 169 (1981)

G. Poletto, R. A. Kopp, in The Lower Atmosphere of Solar Flares; Proceedings of the Solar Maximum Mission Symposium (1986), pp. 453-465, URL http://adsabs.harvard.edu/cgi-bin/nph-bib_query?bibcode=1986lasf .symp. .453P\&db_key=AST

E. R. Priest, T. G. Forbes, Astronomy Astrophysics Revs. 10, 313 (2002)

J. Qiu, D. E. Gary, G. D. Fleishman, Solar Phys. 255, 107 (2009), doi:10.1007/s11207-009-9316-y

J. Qiu, J. Lee, D. E. Gary, Astrophys. J. 603, 335 (2004), doi:10.1086/381353

J. Qiu, J. Lee, D. E. Gary, H. Wang, ApJ 565, 1335 (2002)

R. Ramaty, B. Kozlovsky, R. E. Lingenfelter, Astrophys. Suppl. 40, 487 (1979), doi:10.1086/190596

R. Ramaty, N. Mandzhavidze, in Highly Energetic Physical Processes and Mechanisms for Emission from Astrophysical Plasmas, ed. by P. C. H. Martens, S. Tsuruta, M. A. Weber (2000), volume 195 of IAU Symposium, pp. 123-+

N. D. R. Ranns, S. A. Matthews, L. K. Harra, J. L. Culhane, Astron. Astrophys. 364, 859 (2000)

N. Raouafi, S. K. Solanki, T. Wiegelmann, in Astronomical Society of the Pacific Conference Series, ed. by S. V. Berdyugina, K. N. Nagendra, \& R. Ramelli (2009), volume 405 of Astronomical Society of the Pacific Conference Series, pp. 429-+

F. Reale, G. Peres, Astron. Astrophys. 299, 225 (1995)

D. V. Reames, Space Science Reviews 90, 413 (1999), doi:10.1023/A:1005105831781

K. K. Reeves, H. P. Warren, Astrophys. J. 578, 590 (2002), doi:10.1086/342310

S. Régnier, T. Amari, E. Kersalé, Astron. Astrophys. 392, 1119 (2002), doi:10.1051/0004-6361:20020993

S. Régnier, R. C. Canfield, Astron. Astrophys. 451, 319 (2006), doi:10.1051/0004-6361:20054171

S. Régnier, E. R. Priest, Astron. Astrophys. 468, 701 (2007), arXiv:astro-ph/0703756 doi:10.1051/0004-6361:20077318

T. R. Rimmele, K. Richards, S. L. Hegwer, D. Ren, S. Fletcher, S. Gregory, L. V. Didkovsky, C. J. Denker, W. Marquette, J. Marino, P. R. Goode, in Society of Photo-Optical Instrumentation Engineers (SPIE) Conference Series, ed. by P. L. Wizinowich, D. Bonaccini (2003), volume 4839 of Society of PhotoOptical Instrumentation Engineers (SPIE) Conference Series, pp. 635-646, doi:10.1117/12.457018

P. L. Rochus, J. Defise, J. Halain, C. A. J. Jamar, E. Mazy, L. Rossi, T. Thibert, F. Clette, P. Cugnon, D. Berghmans, J. Hochedez, J. Delaboudiniere, F. Auchere, R. Mercier, M. Ravet, F. Delmotte, M. Idir, U. H. Schuehle, V. Bothmer, S. Fineschi, R. A. Howard, J. D. Moses, J. S. Newmark, in Society of PhotoOptical Instrumentation Engineers (SPIE) Conference Series, ed. by S. Fineschi \& M. A. Gummin (2004), volume 5171 of Society of Photo-Optical Instrumentation Engineers (SPIE) Conference Series, pp. 53-64, doi:10.1117/12.503964

F. Rubio da Costa, L. Fletcher, N. Labrosse, F. Zuccarello, Astron. Astrophys. 507, 1005 (2009), 0909.4705 doi:10.1051/0004-6361/200912651

J. L. R. Saba, T. Gaeng, T. D. Tarbell, Astrophys. J. 641, 1197 (2006), doi:10.1086/500631

P. Saint-Hilaire, A. O. Benz, Solar Phys. 210, 287 (2002), arXiv:astro-ph/0210023 doi:10.1023/A:1022478300679

P. Saint-Hilaire, A. O. Benz, Astron. Astrophys. 435, 743 (2005), doi:10.1051/0004-6361:20041918 
P. Saint-Hilaire, S. Krucker, R. P. Lin, Solar Phys. 250, 53 (2008), doi:10.1007/s11207-008-9193-9

T. Sakao, Characteristics of solar flare hard X-ray sources as revealed with the Hard X-ray Telescope aboard the Yohkoh satellite, Ph.D. thesis, (University of Tokyo), (1994) (1994)

T. Sakao, T. Kosugi, S. Masuda, K. Yaji, M. Inda-Koide, K. Makishima, in Proceedings of Kofu Symposium (1994), pp. 169-172

T. Sakao, T. Kosugi, S. Masuda, K. Yaji, M. Inda-Koide, K. Makishima, Advances in Space Research 17, 67 (1996), doi:10.1016/0273-1177(95)00544-O

T. Sakurai, K. Shibata, K. Ichimoto, S. Tsuneta, L. W. Acton, Pub. Astron. Soc. Japan 44, L123 (1992)

R. Saldanha, S. Krucker, R. P. Lin, Astrophys. J. 673, 1169 (2008), doi:10.1086/524929

P. H. Scherrer, R. S. Bogart, R. I. Bush, J. T. Hoeksema, A. G. Kosovichev, J. Schou, W. Rosenberg, L. Springer, T. D. Tarbell, A. Title, C. J. Wolfson, I. Zayer, MDI Engineering Team, Solar Phys. 162, 129 (1995), doi:10.1007/BF00733429

E. J. Schmahl, M. R. Kundu, V. I. Garaimov, Astrophys. J. 643, 1271 (2006), doi:10.1086/503151

E. J. Schmahl, R. L. Pernak, G. J. Hurford, J. Lee, S. Bong, Solar Phys. 240, 241 (2007), doi:10.1007/s11207007-0263-1

B. Schmieder, T. G. Forbes, J. M. Malherbe, M. E. Machado, Astrophys. J. 317, 956 (1987), doi:10.1086/165344

B. Schmieder, P. Heinzel, J. E. Wiik, J. Lemen, B. Anwar, P. Kotrc, E. Hiei, Solar Phys. 156, 337 (1995)

C. J. Schrijver, Advances in Space Research 43, 739 (2009), 0811.0787 doi:10.1016/j.asr.2008.11.004

C. J. Schrijver, M. L. DeRosa, T. Metcalf, G. Barnes, B. Lites, T. Tarbell, J. McTiernan, G. Valori, T. Wiegelmann, M. S. Wheatland, T. Amari, G. Aulanier, P. Démoulin, M. Fuhrmann, K. Kusano, S. Régnier, J. K. Thalmann, Astrophys. J. 675, 1637 (2008), arXiv:0712.0023 doi:10.1086/527413

C. J. Schrijver, M. L. Derosa, T. R. Metcalf, Y. Liu, J. McTiernan, S. Régnier, G. Valori, M. S. Wheatland, T. Wiegelmann, Solar Phys. 235, 161 (2006), doi:10.1007/s11207-006-0068-7

C. J. Schrijver, M. L. DeRosa, A. M. Title, T. R. Metcalf, Astrophys. J. 628, 501 (2005), doi:10.1086/430733

R. A. Schwartz, B. Dennis, A. K. Tolbert, R. Murphy, G. Share, G. Fishman, M. Briggs, F. Longo, R. Diehl, R. Wijers, in American Astronomical Society Meeting Abstracts \#216 (2010), volume 41 of Bulletin of the American Astronomical Society, pp. \#404.06-+

A. Severny, Space Science Reviews 3, 451 (1964), doi:10.1007/BF00214468

N. R. Sheeley, Jr., R. A. Howard, M. J. Koomen, D. J. Michels, Astrophys. J. 272, 349 (1983), doi: $10.1086 / 161298$

N. R. Sheeley, Jr., H. P. Warren, Y.-M. Wang, Astrophys. J. 616, 1224 (2004), doi:10.1086/425126

K. Shibasaki, Astrophys. J. 557, 326 (2001), doi:10.1086/321651

T. Shimizu, Advances in Space Research 29, 2009 (2002)

A. V. R. Silva, H. Wang, D. E. Gary, Astrophys. J. 545, 1116 (2000), doi:10.1086/317822

A. V. R. Silva, H. Wang, D. E. Gary, N. Nitta, H. Zirin, Astrophys. J. 481, 978 (1997), doi:10.1086/304076

D. M. Smith, R. P. Lin, P. Turin, D. W. Curtis, J. H. Primbsch, R. D. Campbell, R. Abiad, P. Schroeder, C. P. Cork, E. L. Hull, D. A. Landis, N. W. Madden, D. Malone, R. H. Pehl, T. Raudorf, P. Sangsingkeow, R. Boyle, I. S. Banks, K. Shirey, R. Schwartz, Solar Phys. 210, 33 (2002), doi:10.1023/A:1022400716414

B. V. Somov, T. Kosugi, Astrophys. J. 485, 859 (1997)

B. V. Somov, T. Kosugi, H. S. Hudson, T. Sakao, S. Masuda, Astrophys. J. 579, 863 (2002), doi: $10.1086 / 342842$

T. Spirock, C. Denker, H. Chen, J. Chae, J. Qiu, J. Varsik, H. Wang, P. R. Goode, W. Marquette, in Advanced Solar Polarimetry - Theory, Observation, and Instrumentation, ed. by M. Sigwarth (2001), volume 236 of Astronomical Society of the Pacific Conference Series, pp. 65-+

A. C. Sterling, H. S. Hudson, Astrophys. J. Lett. 491, L55+ (1997), doi:10.1086/311043

A. C. Sterling, R. L. Moore, Astrophys. J. 630, 1148 (2005), doi:10.1086/432044

K. T. Strong, A. O. Benz, B. R. Dennis, A. I. Poland, J. W. Leibacher, R. Mewe, J. Schrijver, G. Simnett, J. B. Smith, Jr., J. Sylwester, Solar Phys. 91, 325 (1984)

Y. Su, L. Golub, A. A. Van Ballegooijen, Astrophys. J. 655, 606 (2007), doi:10.1086/510065

Y. N. Su, L. Golub, A. A. van Ballegooijen, M. Gros, Solar Phys. 236, 325 (2006), doi:10.1007/s11207-0060039-z

J. J. Sudol, J. W. Harvey, Astrophys. J. 635, 647 (2005), doi:10.1086/497361

L. Sui, G. D. Holman, Astrophys. J. Lett. 596, L251 (2003), doi:10.1086/379343

L. Sui, G. D. Holman, B. R. Dennis, Astrophys. J. 612, 546 (2004), doi:10.1086/422515

L. Sui, G. D. Holman, B. R. Dennis, Astrophys. J. 626, 1102 (2005), doi:10.1086/430086

P. A. Sweet, ARAA 7, 149 (1969), doi:10.1146/annurev.aa.07.090169.001053

J. Sylwester, I. Gaicki, Z. Kordylewski, M. Kowaliński, S. Nowak, S. Płocieniak, M. Siarkowski, B. Sylwester, W. Trzebiński, J. Bakała, J. L. Culhane, M. Whyndham, R. D. Bentley, P. R. Guttridge, K. J. H. 
Phillips, J. Lang, C. M. Brown, G. A. Doschek, V. D. Kuznetsov, V. N. Oraevsky, A. I. Stepanov, D. V. Lisin, Solar Phys. 226, 45 (2005), doi:10.1007/s11207-005-6392-5

J. Sylwester, J. R. Lemen, R. D. Bentley, A. Fludra, M.-C. Zolcinski, Astrophys. J. 501, 397 (1998), doi: $10.1086 / 305785$

J. Sylwester, J. R. Lemen, R. Mewe, Nature 310, 665 (1984), doi:10.1038/310665a0

S. I. Syrovatskii, ARAA 19, 163 (1981), doi:10.1146/annurev.aa.19.090181.001115

T. Takakura, S. Tsuneta, N. Nitta, K. Makishima, T. Murakami, Y. Ogawara, M. Oda, K. Ohki, S. Miyamoto, Astrophys. J. Lett. 270, L83 (1983), doi:10.1086/184075

F. Tang, Solar Phys. 102, 131 (1985)

M. Temmer, A. M. Veronig, B. Vršnak, C. Miklenic, Astrophys. J. 654, 665 (2007), doi:10.1086/509634

M. Temmer, A. M. Veronig, B. Vršnak, J. Rybák, P. Gömöry, S. Stoiser, D. Maričić, Astrophys. J. Lett. 673, L95 (2008), doi:10.1086/527414

L. Teriaca, A. Falchi, G. Cauzzi, R. Falciani, L. A. Smaldone, V. Andretta, Astrophys. J. 588, 596 (2003), doi: $10.1086 / 373946$

L. Teriaca, A. Falchi, R. Falciani, G. Cauzzi, L. Maltagliati, Astron. Astrophys. 455, 1123 (2006), 0903.0232 doi:10.1051/0004-6361:20065065

J. K. Thalmann, T. Wiegelmann, Astron. Astrophys. 484, 495 (2008), doi:10.1051/0004-6361:200809508

R. J. Thomas, R. G. Teske, Solar Phys. 16, 431 (1971), doi:10.1007/BF00162486

B. J. Thompson, S. P. Plunkett, J. B. Gurman, J. S. Newmark, O. C. St. Cyr, D. J. Michels, Geophys. Res. Lett. 25, 2465 (1998), doi:10.1029/98GL50429

V. S. Titov, E. R. Priest, P. Demoulin, Astron. Astrophys. 276, 564 (1993)

M. Tomczak, Astron. Astrophys. 366, 294 (2001), doi:10.1051/0004-6361:20000204

S. Tomczyk, G. L. Card, T. Darnell, D. F. Elmore, R. Lull, P. G. Nelson, K. V. Streander, J. Burkepile, R. Casini, P. G. Judge, Solar Phys. 247, 411 (2008), doi:10.1007/s11207-007-9103-6

D. Tripathi, H. Isobe, H. E. Mason, Astron. Astrophys. 453, 1111 (2006), doi:10.1051/0004-6361:20064993

S. Tsuneta, L. Acton, M. Bruner, J. Lemen, W. Brown, R. Caravalho, R. Catura, S. Freeland, B. Jurcevich, J. Owens, Solar Phys. 136, 37 (1991)

S. Tsuneta, H. Hara, T. Shimizu, L. W. Acton, K. T. Strong, H. S. Hudson, Y. Ogawara, Pub. Astron. Soc. Japan 44, L63 (1992)

Y. Uchida, Solar Phys. 39, 431 (1974)

S. Ueno, S. Nagata, R. Kitai, H. Kurokawa, in The Solar-B Mission and the Forefront of Solar Physics, ASP Conference Series, Vol. 325, ed. by T. Sakurai, T. Sekii (2004), p. 319

Z. Svestka, Space Science Reviews 5, 388 (1966), doi:10.1007/BF02653250

Z. Švestka, Solar Phys. 13, 471 (1970), doi:10.1007/BF00153567

Z. Švestka, P. Hoyng, W. van Tend, A. Boelee, C. de Jager, R. T. Stewart, L. W. Acton, E. C. Bruner, A. H. Gabriel, C. G. Rapley, C. de Jager, H. LaFleur, G. Nelson, G. M. Simnett, H. F. van Beek, W. J. Wagner, Solar Phys. 75, 305 (1982), doi:10.1007/BF00153479

Z. F. Svestka, J. M. Fontenla, M. E. Machado, S. F. Martin, D. F. Neidig, Solar Phys. 108, 237 (1987)

H. F. van Beek, C. de Jager, A. Schadee, Z. Svestka, A. Boelee, A. Duijveman, M. Galama, R. Hoekstra, P. Hoyng, R. Fryer, Astrophys. J. Lett. 244, L157 (1981), doi:10.1086/183502

G. H. J. van den Oord, Astron. Astrophys. 234, 496 (1990)

J. E. Vernazza, E. H. Avrett, R. Loeser, Astrophys. Suppl. 45, 635 (1981), doi:10.1086/190731

A. Veronig, B. Vršnak, B. R. Dennis, M. Temmer, A. Hanslmeier, J. Magdalenić, Astron. Astrophys. 392, 699 (2002a), arXiv:astro-ph/0207217 doi:10.1051/0004-6361:20020947

A. Veronig, B. Vršnak, M. Temmer, A. Hanslmeier, Solar Phys. 208, 297 (2002b), arXiv:astro-ph/0208088

A. M. Veronig, J. C. Brown, Astrophys. J. Lett. 603, L117 (2004), doi:10.1086/383199

A. M. Veronig, J. C. Brown, B. R. Dennis, R. A. Schwartz, L. Sui, A. K. Tolbert, Astrophys. J. 621, 482 (2005), doi:10.1086/427274

A. M. Veronig, M. Karlický, B. Vršnak, M. Temmer, J. Magdalenić, B. R. Dennis, W. Otruba, W. Pötzi, Astron. Astrophys. 446, 675 (2006), doi:10.1051/0004-6361:20053112

N. Vilmer et al., Space Sci. Rev. pp. XXX-XXX (2011)

E. Vogt, J. Hénoux, Astron. Astrophys. 349, 283 (1999)

O. von der Luehe, Astron. Astrophys. 268, 374 (1993)

A. Vourlidas, D. Buzasi, R. A. Howard, E. Esfandiari, in ESA Special Publication, ed. by J. Kuijpers (2002), volume 506 of ESA Special Publication, pp. 91-94

A. Vourlidas, P. Subramanian, K. P. Dere, R. A. Howard, Astrophys. J. 534, 456 (2000), doi:10.1086/308747

A. Vourlidas, S. T. Wu, A. H. Wang, P. Subramanian, R. A. Howard, Astrophys. J. 598, 1392 (2003), arXiv:astro-ph/0308367 doi:10.1086/379098

B. Vršnak, E. W. Cliver, Solar Phys. 253, 215 (2008), doi:10.1007/s11207-008-9241-5 
B. Vršnak, M. Temmer, A. Veronig, M. Karlický, J. Lin, Solar Phys. 234, 273 (2006), doi:10.1007/s11207006-0093-6

H. Wang, Solar Phys. 140, 85 (1992)

H. Wang, M. W. Ewell, Jr., H. Zirin, G. Ai, Astrophys. J. 424, 436 (1994), doi:10.1086/173901

H. Wang, D. E. Gary, H. Zirin, R. A. Schwartz, T. Sakao, T. Kosugi, K. Shibata, Astrophys. J. 453, 505 (1995), doi:10.1086/176411

H. Wang, C. Liu, J. Qiu, N. Deng, P. R. Goode, C. Denker, Astrophys. J. Lett. 601, L195 (2004), doi: $10.1086 / 382188$

T. Wang, L. Sui, J. Qiu, Astrophys. J. Lett. 661, L207 (2007), doi:10.1086/519004

Y. Wang, J. Zhang, Astrophys. J. 665, 1428 (2007), 0808.2976 doi:10.1086/519765

H. P. Warren, A. D. Warshall, Astrophys. J. Lett. 560, L87 (2001), doi:10.1086/324060

H. P. Warren, A. R. Winebarger, P. S. Hamilton, Astrophys. J. Lett. 579, L41 (2002), doi:10.1086/344921

D. G. Wentzel, Astrophys. J. 208, 595 (1976)

M. S. Wheatland, Astrophys. J. 609, 1134 (2004), arXiv:astro-ph/0403613 doi:10.1086/421261

M. S. Wheatland, D. B. Melrose, Solar Phys. 158, 283 (1995)

S. M. White, T. S. Bastian, R. Bradley, C. Parashare, L. Wye, in Astronomical Society of the Pacific Conference Series, ed. by N. Kassim, M. Perez, W. Junor, P. Henning (2005a), volume 345 of Astronomical Society of the Pacific Conference Series, pp. 176-+

S. M. White, R. J. Thomas, R. A. Schwartz, Solar Phys. 227, 231 (2005b), doi:10.1007/s11207-005-2445-z

S. White et al., Space Sci. Rev. pp. XXX-XXX (2011)

E. Wiehr, G. Stellmacher, Astron. Astrophys. 247, 379 (1991)

J. M. Wilcox, A. J. Hundhausen, J. Geophys. Res. 88, 8095 (1983), doi:10.1029/JA088iA10p08095

J. P. Wild, S. F. Smerd, A. A. Weiss, ARAA 1, 291 (1963), doi:10.1146/annurev.aa.01.090163.001451

K. Wilhelm, W. Curdt, E. Marsch, U. Schühle, P. Lemaire, A. Gabriel, J.-C. Vial, M. Grewing, M. C. E. Huber, S. D. Jordan, A. I. Poland, R. J. Thomas, M. Kühne, J. G. Timothy, D. M. Hassler, O. H. W. Siegmund, Solar Phys. 162, 189 (1995), doi:10.1007/BF00733430

B. E. Woodgate, R. A. Shine, A. I. Poland, L. E. Orwig, Astrophys. J. 265, 530 (1983), doi:10.1086/160696

T. N. Woods, F. G. Eparvier, J. Fontenla, J. Harder, G. Kopp, W. E. McClintock, G. Rottman, B. Smiley, M. Snow, Geophys. Res. Lett. 31, 10802 (2004), doi:10.1029/2004GL019571

T. N. Woods, F. G. Eparvier, R. Hock, A. R. Jones, D. Woodraska, D. Judge, L. Didkovsky, J. Lean, J. Mariska, H. Warren, D. McMullin, P. Chamberlin, G. Berthiaume, S. Bailey, T. Fuller-Rowell, J. Sojka, W. K. Tobiska, R. Viereck, Solar Phys. pp. 3-+ (2010), doi:10.1007/s11207-009-9487-6

T. N. Woods, G. Kopp, P. C. Chamberlin, Journal of Geophysical Research (Space Physics) 111, 10 (2006), doi:10.1029/2005JA011507

J.-P. Wuelser, R. C. Canfield, L. W. Acton, J. L. Culhane, A. Phillips, A. Fludra, T. Sakao, S. Masuda, et al., Astrophys. J. 424, 459 (1994), doi:10.1086/173903

Y. Xu, W. Cao, C. Liu, G. Yang, J. Qiu, J. Jing, C. Denker, H. Wang, Astrophys. J. Lett. 607, L131 (2004), doi:10.1086/422099

Y.-H. Yang, C. Z. Cheng, S. Krucker, R. P. Lin, W. H. Ip, Astrophys. J. 693, 132 (2009), doi:10.1088/0004$637 \mathrm{X} / 693 / 1 / 132$

T. Yokoyama, K. Akita, T. Morimoto, K. Inoue, J. Newmark, Astrophys. J. Lett. 546, L69 (2001), doi: $10.1086 / 318053$

P. R. Young, G. Del Zanna, E. Landi, K. P. Dere, H. E. Mason, M. Landini, Astrophys. Suppl. 144, 135 (2003), arXiv: astro-ph/0209493 doi:10.1086/344365

D. M. Zarro, R. C. Canfield, T. R. Metcalf, K. T. Strong, Astrophys. J. 324, 582 (1988), doi:10.1086/165919

D. M. Zarro, J. R. Lemen, Astrophys. J. 329, 456 (1988), doi:10.1086/166391

J. Zhang, K. P. Dere, R. A. Howard, M. R. Kundu, S. M. White, Astrophys. J. 559, 452 (2001), doi: $10.1086 / 322405$

J. Zhang, K. P. Dere, R. A. Howard, A. Vourlidas, Astrophys. J. 604, 420 (2004), doi:10.1086/381725

V. V. Zharkova, Solar Phys. 251, 641 (2008), doi:10.1007/s11207-008-9216-6

V. V. Zharkova, S. I. Zharkov, Astrophys. J. 664, 573 (2007), 0802.2787. doi:10.1086/518731

V. V. Zharkova et al., Space Sci. Rev. pp. XXX-XXX (2011)

T. Zhou, H. Ji, G. Huang, Advances in Space Research 41, 1195 (2008), doi:10.1016/j.asr.2007.04.095

H. Zirin, M. A. Liggett, Solar Phys. 113, 267 (1987)

H. Zirin, K. Tanaka, Solar Phys. 32, 173 (1973), doi:10.1007/BF00152736 


\section{Index}

abundances, 62

coronal, 61

$\mathrm{Fe} / \mathrm{H}, 62,65$

acceleration, 60

and CME acceleration, 58

low-energy cutoff, 8, 65, 69

SEPs, 19, 70

stochastic, 49

ACRIM, 66

active regions

and flare occurrence, 4

adiabatic compression, 43

Advanced Composition Explorer (ACE), 67

Alfvén speed

and downflows, 13

middle corona, 60

variation with height

illustration, 60

arcade, 4, 12, 13, 22, 28, 29, 34, 53

growth, 53

illustration, 4, 11, 26

magnetic structure, 55

backwarming, 40, 41

Balmer- $\alpha$

impact polarization, 71

Bayesian approach, 5

beams

at photosphere, 39

proton, 39, 41

vs. conduction fronts, 25

big-flare syndrome, 22

bound-bound emission, 62

bremsstrahlung

thin-target, 52

cartoons, 72

Giovanelli, 73

omissions, 74

standard, 73

causality, 5

caveats

photospheric field extrapolation, 31, 68

CCD, 18 charge exchange, 71

Chianti, 61, 63, 64

energetics, 68

$\mathrm{Fe} / \mathrm{Fe}-\mathrm{Ni}$ ratio, 63

chromosphere

flare-related flows, 9

chromospheric condensation, 43

chromospheric evaporation, $8,11,42$

conduction-driven, 22

explosive, 43,44

illustration, 44

gentle, 43,45

illustration, 45

imaging, 46

temperature-dependent flows, 45

chromospheric heating, 43, 46

in flares, 43

collapsing magnetic trap, 30

CoMP, 38, 71

conduction

thermal, 11

conduction fronts, 45

conjugacy, 22, 54

continuum

Balmer, 39, 40

$\gamma$-ray pseudo-continuum, 69

$H^{-}, 39$

non-thermal electron, 65

Paschen, 39

soft X-ray, 61

two-photon, 61

cooling

and energy estimates, 68

and shrinkage, 54

conductive, 11

equilibrium, 64

parameter dependence, 11

radiative, 11,68

coronal dimmings, 57

coronal heating

and reconnection, 72

chromospheric, 42

in flare-like events, 3

coronal mass ejections (CMEs), 6, 8, 55,

57 
acceleration, 58

acceleration and flare energy release, 58

and coronal sources, 52

and SEP energy

illustration, 70

energy partition, 68

illustration, 53

velocity field, 68

coronal seismology, 77

coronal sources, 47,52

contraction, 54

current-sheet morphology, 73

double, 50

hard X-ray footpoints, 52

thick-target

illustration, 47

coronal thick target, 46,47

microwave imaging, 48

cosmic rays

solar, 19

Cowling T. G., 73

CSHKP, 12, 13, 24, 29, 48, 50

current sheets, 73

X-ray observations, 49

difference images, 58

dipolarization, 10, 14, 74

distribution functions

flare occurrence, 3

double counting, 66

downflows, 13, 41

coronal rain, 11

evaporative, 44

high temperature, 46

supra-arcade, 10, 13, 50, 76

Dungey, J. W., 73

EIT wave, 59

electric fields

and reconnection rate, 36

convective, 10, 37

parallel, 37

electrons

collisional losses, 35,52

dominant tail population, 52

emission measure, 68

by $G O E S$ class, 3

definition, 68 differential, 64

non-thermal, 75

radio and $\mathrm{SXR}, 43$

energetic neutral atoms, 71

equivalent width, 61,62

eras

RHESSI, 1, 16, 66

Skylab, 31

Yohkoh, 27

fables

blind man and elephant, 56

Fe lines, 51, 61

equivalent width, 61

and RHESSI attenuator state, 62

low ionization states, 61

filaments, 5

activation, 5

filling factor, 68,69

flare (individual)

SOL1859-09-01T11:18 (pre-GOES), 38

SOL1969-03-30T02:47 (pre-GOES), 52

SOL1972-08-04 (pre-GOES) energetics, 67

SOL1973-02-05 (pre-GOES)

energetics, 67

SOL1980-03-29T09:18 (M1), 24

SOL1980-08-31T12:49 (M2.8)

energetics, 67

SOL1992-01-13T17:25 (M2.0), 24

SOL1999-03-18T08:31 (M3.3)

illustration, 11

SOL2000-07-14T10:24 (X5.7), 27

SOL2001-04-10T05:26 (X2.3)

illustration, 11, 26

SOL2001-08-25T16:45 (X5.3), 33

illustration, 32, 41

white light, 34

SOL2001-10-19T01:05 (X1.6)

illustration, 4

SOL2002-02-26T10:27 (C9.6)

energetics, 67

SOL2002-03-14T01:44 (M5.7)

footpoint motion, 29

SOL2002-04-15T00:15 (M3.7)

coronal thick target, 47

illustration, 47 
shrinking loops, 54

SOL2002-04-21T01:51 (X1.5)

$\gamma$-ray upper limits, 70

CME energetics, 68

energetics, 66, 67

illustration, 53

SEPs, 70

thermal plasma, 68

SOL2002-04-30T08:22 (M1.3)

current sheet, 50

illustration, 49, 50

SOL2002-05-31T00:16 (M2.4)

Fe feature, 62

illustration, 62

SOL2002-07-15T11:55 (C9.1), 45

SOL2002-07-17T07:13 (M8.5)

TRACE, 23

illustration, 23

SOL2002-07-23T00:35 (X4.8)

bimodal DEM distribution, 65

CME energetics, 68

energetics, 66, 67

Fe line complex, 51

hard X-ray precursor, 51

magnetic containment, 51

SEPs, 70

soft X-ray line emissions, 63

superhot component, 51

thermal energetics, 68

SOL2002-09-09T17:52 (M2.1)

footpoint motions, 29

SOL2002-09-29T06:39 (M2.6), 40

SOL2002-09-30T01:50 (M2.1)

illustration, 35

white light, 34

SOL2002-10-04T05:38 (M4.0), 10

SOL2002-11-02T06:07 (C3.9)

current sheet, 50

SOL2002-11-09T13:23 (M4.9)

footpoint convergence, 29

footpoint motion, 29

illustration, 29

SOL2003-04-24T15:53 (C8.2)

current sheet, 50

SOL2003-04-26T03:06 (M2.1), 62

illustration, 62

SOL2003-06-10T14:36 (M2.2), 44

SOL2003-10-28T11:10 (X17.2)

TSI detection, 66
SOL2003-10-29T20:49 (X10.0), 6, 35,37

egression power, 40

footpoint motion, 29

illustration, 36, 37, 40

SOL2003-11-03T09:55 (X3.9), 6

illustration, 54, 56

SOL2003-11-04T19:53 (X17.4), 6

SOL2003-11-0T309:55 (X3.9)

current sheet, 50

SOL2003-11-13T05:01 (M1.6)

evaporation, 46

illustration, 46

SOL2003-11-18T08:31 (M3.9)

flux transfer, 37

illustration, 39

SOL2004-11-01T03:22 (M1.1)

footpoint motions, 29

illustration, 30

SOL2005-01-17T09:52 (X3.8), 33

CME, 58

illustration, 12, 34, 58

SOL2005-01-19T08:22 (X1.3) illustration, 52

SOL2005-01-20T07:01 (X7.1) coronal hard X-ray source, 52 illustration, 52

SOL2005-05-13T16:57 (M8.0), 27 hard X-ray ribbons, 37

illustration, 28

SOL2006-07-06T08:36 (M2.5) CME, 58

SOL2006-12-05T10:35 (X9) energetic neutral atoms, 20

SOL2006-12-06T18:47 (X6.5) illustration, 59

SOL2007-12-14T14:16 (C1.1) blueshifts, 45

SOL2007-12-31T01:11 (C8.3) illustration, 57

flare classification, 3

$\mathrm{H} \alpha, 3$

table, 3

flare energetics, 66

flare kernels, 25

$\mathrm{H} \alpha$

illustration, 30

on iso-Gauss contours, 33

UV, 27 
white light, 41

flare models

and cartoons, 72

CSHKP, 12, 13, 24, 29, 48, 50

magnetic mirror, 35

multi-strand, 13

observational constraints, 71

predictive power, 72

radiation hydrodynamic, 24, 43

slip-running reconnection, 29, 33

standard, 72, 73

summary, 71

thick-target, 71

flare types

filament eruption, 5

long-decay events (LDEs), 6

long-duration, 7, 52, 53, 64

spotless, 5

white light, 22, 38

illustration, 10, 34

flares

and CMEs, 55

and coronal dimming, 55

and SEPs, 55

bolometric measurements, 66

Carrington, 38

classification, 3

$\mathrm{H} \alpha, 3$

table, 3

CMEless, 76

coronal manifestations, 9

energetics, 66

double counting, 66

table, 67

energy content

accelerated electrons, 69

accelerated ions, 69

CME, 68

magnetic, 2, 5, 9, 32, 55, 68

overall, 67

partition, 66

solar energetic particles, 70

thermal plasma, 68

energy release and CME dynamics, 57

energy storage, 5,72

GOES classification, 3

hard X-ray precursor, 8, 51

impulsive phase, 9 negative, 43

overview

illustration, 4

phases, 6

pre-flare coronal density, 47

pre-flare filament motions, 5

pre-flare phase, 8

precursor events, 8

total irradiance, 39

white light, 22, 39

flux emergence, 9

flux transfer, 22

footpoints, 22, 24, 29

$\gamma$-rays, 22

and TRACE diffraction patterns, 22

and $\mathrm{H} \alpha$ ribbons, 33

and reconnection rate, 36

and UV ribbons, 37

apparent motions

illustration, 29

asymmetry, 35

and microwaves, 35

statistics, 35

converging motions, 29,54

model requirements, 35

motions, 28

non-thermal emission measure, 75

occultation, 51, 65

parallel motions, 29

separation correlated with HXRs, 54

stationary hot component, 43

vs. ribbons, 9

white light, 22

Fraunhofer lines

$\mathrm{G}$ band, 16

$\mathrm{H} \alpha, 5$

impact polarization, 71

$\mathrm{H} \beta, 17$

Ni I $6758 \AA, 17$

free energy, 2

free-bound emission, 61

non-thermal, 65,71

thermal, 61

free-free emission, 61

frequency

Larmor, 4, 13

plasma, 14

starting, 60

frequency synthesis, 15 
gamma-rays

and seismic waves, 41

and white-light flares, 39

Giovanelli, R. G., 73

global waves, 58

and X-ray dimming, 59

EIT, 59

Moreton, 59

seismic, 23, 40

and backwarming, 41

and Lorentz force, 41

and shock waves, 41

type II radio burst, 52, 58

GOES

energetics, 68

SXI, 54

GOES classification, 3

gradual phase, 4, 11

ground-level events, 20

gyrosynchrotron emission illustration, 4

Hanle effect, 37, 38

hard X-rays

and EUV, 22

and polarity inversion line, 29

and UV ribbons

illustration, 23

and white light, 22

conjugacy, 22

coronal sources, 7,47

coronal thick target, 46, 47

microwaves, 48

definition, 18

footpoint sources, 22

footpoint sources in gradual events, 52

footpoints, 9, 22

gradual events, 7

identified with flare kernels, 25

imaging spectroscopy, 19

multi-wavelength context, 3

need for sensitive observations, 76

non-focusing optics, 19

ribbon-like structures, 27

ribbons, 27, 37

short time scales, 7, 24

soft-hard-harder, 52, 60

soft-hard-soft, 7, 52 sources uncorrelated with UV/EUV, 27

time-of-flight analysis, 24

imaging spectroscopy, 19, 20

impulsive phase, 4, 9, 31

in situ

comparisons with remote sensing, 19, 73

interplanetary coronal mass ejections (ICMEs), 20,59

ionization equilibrium, 61

uncertainties, 64

ionization state, 61

jerks, 41

looptop sources, $9,35,43,46,49,53,54$

downward motions, 54

impulsive phase, 7

thick target, 47

low-energy cutoff, 8, 51, 65, 71

Lumogen, 18

Lyman- $\alpha$

Doppler shifts, 71

need for observations, 76

magnetic charge topology, 32, 34

magnetic field, 13

coronal, 15,38

and plasma containment, 51, 69

energy storage, 9,72

extrapolation, 72

worries about, 31

flare-related changes, 14, 23, 42

illustration, 41

flux annihilation, 73

free energy, 2, 42, 72

height dependence, 55

jerk, 42

measurement

radio techniques, 13

potential, 13

shear distribution, 32

magnetic storm, 59

magnetic structures

2.5-D approximation, 36

and coronal seismology, 77

bald patch, 13 
CME formation, 8

core fields, 72

coronal current sheets, 73

coronal trap, 52

current sheet, 50

and double coronal sources, 49

cusps, 9

illustration, 11

$\delta$ spot, 5,13

illustration, 5

dipolarization, 74

energy storage, 9,13

extrapolation

magnetic charge topology, 32

extrapolations from photosphere caveats, 68

force-free, 9

force-free arcade, 55

fractal dimension, 5

implosion, 54

long polarity inversion lines, 5

moss, 25

multi-strand loops, 13

need for coronal magnetography, 37

neutral line, 4, 13, 22

null, 31,34

polarity inversion line, 4, 28, 72

quadrupolar configuration, 25

quasi-separatrix layers, 22

separatrices, 10, 22, 31, 32

illustration, 32

separatrices and flare ribbons, 32

sheared arcade, 55

shrinkage, 53

strong gradients, 5

sunspots

field topology, 10

three-dimensionality, 10

turbulent particle confinement, 49

twists

and flare prediction, 5

variable shear, 5

magnetosphere

terrestrial, 59

Masuda flare, 24, 47

microflares, 62

models

non-LTE, 40

radiation hydrodynamic, 27 momentum balance, 44

Moreton wave, 59

moss, 25

Neupert effect, 7, 42, 44

and CME acceleration phase, 58

illustration, 46

use in place of HXRs, 44

neutron emission, 71

neutron monitors, 20

neutron telescopes, 20

neutrons, 19,76

half-life, 20

need for low-energy observations, 76

non-isothermality, 64

non-potential energy, 5, 13

observatories

Advanced Technology Solar Telescope (ATST), 16

Big Bear, 16

Catania, 16

Dunn Solar Telescope, 16

Dutch Open Telescope, 16

El Leoncito, 15

European Solar Telescope (EST), 16

FASR, 15, 37

German Vacuum Tower Telescope, 16

GONG, 17

Green Bank, 14

Hida, 16, 17

Hiraiso, 15

Holloman, 16

Huairou, 17

Kanzelhöhe, 16

Kharkov, 16

Learmonth, 16

LOFAR, 15

MSFC, 17

Nançay, 14

Nobeyama, 14, 48

Owens Valley Solar Array (OVSA), 14

Phoenix-2, 15

Pic du Midi, 16

Purple Mountain, 15, 16

Multichannel Infrared Solar Spectrograph (MISS), 15 
San Vito, 16

SOLIS, 15, 17

Swedish Solar Observatory, 16

Themis, 16

Tremsdorf, 15

Yunnan, 16

observing facilities, 14

$\gamma$-rays, 19

EUV, 18

$\mathrm{H} \alpha, 16$

hard X-rays, 19

infrared, 15

magnetographs, 16

neutrons, 20

optical, 15

particles and fields, 19

radio, 14

soft X-rays, 18

solar energetic particles, 19

ultraviolet, 17

occulted sources, 51

exponential decay, 52

illustration, 52

impulsive phase, 52

opacity minimum, 15, 23

Owens Valley Solar Array (OVSA), 4

Parker, E. N., 73

Petschek, H. E., 73

plasma instabilities

bump-on-tail, 24

high beta, 49

loss-cone, 49, 52

and soft-hard-harder pattern, 49

plasma turbulence, 8,74

polarization

circular, 48

$\mathrm{H} \alpha, 71$

Poynting flux

and reconnection, 37

estimate from footpoint motions, 37

precursor, 8

hard X-ray, 51

superhot, 51

protons

beams, 39

quasi-periodic pulsations, 7

quasi-separatrix structure, 22 radiation hydrodynamics, 43

and beam energy, 27

radiative loss function, 68

radio emission

microwaves

circular polarization, 48

need for imaging spectroscopy, 76

negative microwave bursts, 43

Razin suppression, 24

spectrograms, 24

type II burst, 59

type III burst, 6, 60

Razin effect, 24

reactions

charge-exchange, 71

recombination radiation, 39, 40, 61

recombination radiation (non-thermal), 65

reconnection, 9

and HXR footpoints, 33

circumstantial evidence for, 11

convective electric field, 10

flux transfer, 22

flux transfer in ribbons, 36

footpoint motions, 28, 37

gradual phase, 11

history, 73

illustration, 37

impulsive phase, 9

inflow, 73

microphysics vs global physics, 73

non-occurrence, 72

outflow, 50

slip-running, 29

standard flare model, 12, 13, 24, 29, 48,50

vs. annihilation, 73

return current

and beam stability, 75

RHESSI

spectral response matrix, 63

RHESSI, 3

and QPP, 7

attenuating shutters, 62

configuration, 19

$\gamma$-ray imaging, 22

imaging spectroscopy, 64

ribbons, 4, 24

and separatrices, 10

and shear, 22 
early vs. gradual phase, 24

energy release rate, 34

EUV, 22

flux transfer, 36

illustration, 37

$\mathrm{H} \alpha, 12$

$\mathrm{H} \alpha, 10,22$

illustration, 25, 32

hard X-ray, 27

illustration, 28

high current density, 32

illustration, 10, 23

motions, 22, 28

multiple, 71

physics, 22

topology, 22

TRACE EUV observations

illustration, 25

UV, 10, 22, 27

vs. footpoints, 9

rotating modulation collimator, 19

satellites

$A C E, 20,66,67$

CGRO

BATSE, 7

CORONAS-F

RESIK, 18

GOES, 4

GOES, 3, 66

SXI, 58

GSAT-2

SOXS, 66

Hinode, 16-18, 26

EIS, 42, 45

SOT, 16, 42

SP, 17

XRT, 18, 47

INTEGRAL, 19

OSO-5, 47, 52

OSO-7, 47

RHESSI, 16

SDO, 17, 18

AIA, 18

EVE, 19

$S M M$

ACRIM, 66

BCS, 62

HXRBS, 7
$\mathrm{SOHO}$

LASCO, 68

SOHO, 18, 20, 66, 67

CDS, 42

EIT, 29, 64

LASCO, 58

MDI, 16, 42

SORCE

TIM, 66

STEREO, 18, 20, 71

TD-1A, 52

TRACE , 5, 16, 17, 19, 22

Ulysses, 20

WIND, 20

Yohkoh, 4

Yohkoh, 7, 47

BCS, 18

HXT, 19

SXT, 18

white-light observations, 16

scattering

inelastic, 23

Thomson, 68

separatrix, 10, 22, 31, 33

illustration, 32

shocks

and Moreton waves, 59

CME-driven, 59

hydrodynamic, 41

interplanetary, 59

particle acceleration, 60,70

radiative damping, 41

spectrogram signature, 59

sunquake excitation, 41

type II radio burst, 58

simulations

non-LTE radiative transfer, 40

radiation hydrodynamic, 8,13

Skylab, 17

soft X-rays

continuum, 61

definition, 18

double coronal source

illustration, 50

double coronal sources, 49, 73

emission lines, 18

emission measure, 63

Fe K-shell emission, 61

Fe lines, 62 
gradual time profiles, 6

long-decay events (LDE), 6, 53, 64

moss, 25

ribbons, 4

spectroscopy, 61

summary of RHESSI results, 65

superhot component, 51

temperature coverage, 18

soft-hard-harder, 7, 52, 60

soft-hard-soft, 7, 52

$\mathrm{SOHO}$

CDS, 44

Solar and Heliospheric Observatory (SOHO), 67

solar energetic particles (SEPs), 19, 60

total energy content, 70

specific ionization approximation, 40

spectrum

electrons

and efficiency of evaporation, 46 flare

broad-band nature of, 2

$\gamma$-ray lines, 69

$\gamma$-ray pseudo-continuum, 69

infrared, 15

neutron-decay products, 20

$\pi^{0}$-decay, 14

SXR continuum

and emission lines, 51

SXR emission lines, 62

standard model, 28, 29

2-D, 31

stereoscopic observations, 20

HXRs, 52

storm sudden commencement, 59

submillimeter emission

observatories, 15

SUMER, 17

sunquakes, 40

and proton beams, 41

sunspots

and flare ribbons, 33

illustration, 34

and free magnetic energy, 2

and seismic sources, 41

illustration, 40

and white-light emission

illustration, 10

connectivity, 10 $\delta$ spots, 5,13

penumbral disappearance, 42

umbral field, 14

superhot component, 51

suprathermal populations, 74

Sweet, P. A., 73

syndromes

big-flare, 22

temperature minimum, 40

thermal conduction, 11

thick-target model, 22

collisional, 3, 44

time-of-flight analysis, 24

Total Irradiance Monitor (TIM), 66

TRACE

and DEM, 64

flare arcade in EUV

illustration, 11

flare UV kernels, 27

UV correlation with HXRs, 27

UV footpoints, 37

UV ribbons

illustration, 10

white light, 33

transition region, 18, 27

and evaporation, 43

trapping

coronal, 52

magnetic, 52

TSI, 66

tsunami, 40, 59

turbulence, 8

(u,v)-plane, 15

upflows, 11, 13, 42, 44, 46

and beam heating, 45

EUV, 9

explosive, 44

height limit, 43

SXR, 9

temperature dependence, 45

wave-particle interactions, 52

waves

energy transport, 24, 41, 42

quasi-periodic pulsations, 7

sausage mode, 7

seismic, 23 
and $\gamma$-rays, 41

white-light flares, 34, 38, 40

energy input, 34

illustration, 35

sub-arcsecond structure, 26

type I, 39

type II, 39

XUV spectral range, 19

Zeeman effect, 13

infrared, 37 